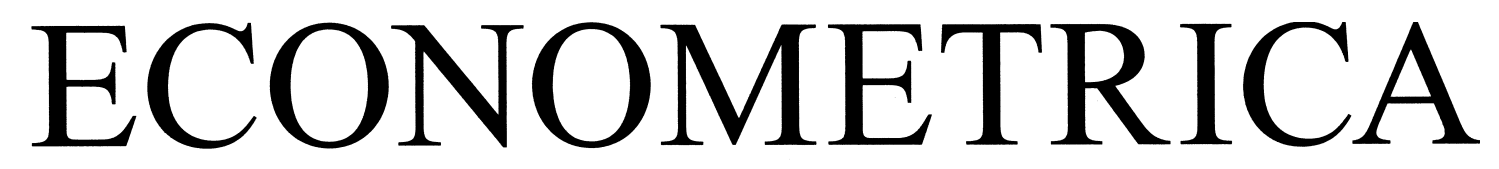

JOURNAL OF THE ECONOMETRIC SOCIETY

An International Society for the Advancement of Economic

Theory in its Relation to Statistics and Mathematics

http://www.econometricsociety.org/

Econometrica, Vol. 87, No. 5 (September, 2019), 1693-1762

MARKET SELECTION WITH DIFFERENTIAL FINANCIAL CONSTRAINTS

ANI GUERDJIKOVA

GAEL, University de Grenoble Alpes

JOHN QUIGGIN

School of Economics, Faculty of Business, Economics, and Law, University of Queensland

The copyright to this Article is held by the Econometric Society. It may be downloaded, printed and reproduced only for educational or research purposes, including use in course packs. No downloading or copying may be done for any commercial purpose without the explicit permission of the Econometric Society. For such commercial purposes contact the Office of the Econometric Society (contact information may be found at the website http://www.econometricsociety.org or in the back cover of Econometrica). This statement must be included on all copies of this Article that are made available electronically or in any other format. 


\title{
MARKET SELECTION WITH DIFFERENTIAL FINANCIAL CONSTRAINTS
}

\author{
ANI GUERDJIKOVA \\ GAEL, University de Grenoble Alpes
}

JOHN QUIGGIN

School of Economics, Faculty of Business, Economics, and Law, University of Queensland

\begin{abstract}
We analyze financial markets in which agents face differential constraints on the set of assets in which they can trade. In particular, the assets available to each agent span a partition of the state space that can be strictly coarser than the partition spanned by the assets available in the market. We first show that the existence of differential constraints has an impact on prices and allocations as compared to a complete financial market with unconstrained agents.

We consider the implications for survival, taking the work of Blume and Easley (2006) as a starting point. We show that whenever agents have identical correct beliefs and equal discount factors, and their partitions are nested, all agents survive. When agents have heterogeneous beliefs, differential constraints may allow agents with wrong beliefs to survive. Provided constraints are relevant (in a sense we define more precisely), the condition for an agent to survive is that his survival index is at least as large as that of the agents with finer partitions. We also study the impact of deregulation (an increase in the set of assets available to some agents). Unless the agent can adopt beliefs that are closer to the truth on the newly refined partition than those of less constrained agents, increasing his opportunities for trade might harm his chances for survival.
\end{abstract}

KEYWORDS: Differential financial constraints, asset markets, survival.

\section{INTRODUCTION}

THE QUESTION OF WHETHER FINANCIAL MARKETS PRICE ASSETS ACCURATELY IS of central importance in economics, especially in the light of the rapid increase in the volume, value, and complexity of financial transactions over recent decades. The strong form of the efficient markets hypothesis (EMH) states that the market price of an asset is the best possible estimate of its value, given all available information, public and private. However, the observed behavior of financial markets appears to be inconsistent with the strong-form EMH in a number of respects. Notable examples are excessive volatility (including apparent "bubbles" and crashes), and the "equity premium" and "risk-free rate" puzzles.

One argument in favor of strong-form EMH, discussed by Blume and Easley (2006) and Sandroni (2000), is the idea that markets favor the best informed and most rational traders. Trades in a financial market may be seen as "betting one's beliefs" about the relative probabilities of different states of nature and the resulting returns on assets. Over time, traders who correctly judge these probabilities and make rational investment choices based on their beliefs will accumulate wealth at the expense of others. In the limit,

Ani Guerdjikova: ani.guerdjikova@univ-grenoble-alpes.fr

John Quiggin: j.quiggin@uq.edu.au

This research was supported by IUF, by Labex MME-DII, and by an Australian Research Council Laureate Felllowship. We would like to thank four anonymous referees, Levon Barseghyan, Pablo Beker, Christophe Bravard, Rose-Anne Dana, Gabriel Desgranges, David Easley, Giorgio Fabbri, Stefan Gerdjikov, Kristoffer Nimark, Marcus Pivato, Stéphane Sémirat, and Nancy Wallace, as well as seminar participants at the University of Cergy, Cornell University, and Royal Holloway for helpful comments and suggestions. 
only these rational well informed traders will survive, and market prices will reflect their beliefs.

This argument is intuitively appealing, and the central result can be derived under relatively weak conditions. However, the argument raises some serious difficulties.

First, in simple versions of the model, all but the best informed traders vanish almost surely (a.s.). This appears to be inconsistent with observed outcomes, where some traders do better than others over the long run, but poorly informed traders manage to survive. The result is also problematic with regard to welfare. While trade ensures that assets are priced correctly, it does so by greatly increasing consumption inequality. In the limit, consumption is driven to zero for all but the best informed. The empirical failure of predictions on survival, derived from standard models, casts doubt on all the predictions of those models. Hence, it is of interest to consider more realistic models, where poorly informed agents can survive.

Second, in the Blume-Easley version of the model, all traders can select their most preferred portfolio from a set of securities that spans the state space. Hence, traders can be regarded as choosing state-contingent consumption streams, and there is no need to make the associated asset markets explicit.

This assumption is technically convenient and provides insight into the intuition underlying the key results. However, it is problematic because the set of financial assets traded in markets, while it may be large, is finite. By contrast, the set of economically relevant contingencies on which agents might conceivably trade is effectively unbounded.

Even within the span of the market, the assumption that traders can always select their most preferred state-contingent consumption stream is not satisfied by large numbers of agents. Some institutional investors are constrained by requirements to invest only in particular classes of assets or to hold only investments with an investment grade (typically credit rating).

In addition to explicit constraints, the portfolio choices of many investors appear to reflect implicit constraints. These choices may be explained by bounded rationality, for example, in the form of limited awareness (Guiso and Jappelli (2005), Quiggin and Siddiqi (2015))) or as the result of transaction costs associated with entering a particular market as in Blume, Cogley, Easley, Sargent, and Tsyrennikov (2018). Whatever the cause, the result is that the spanning assumption of Blume and Easley becomes problematic. The consumption plans available to investors are restricted to those that can be implemented using the set of assets in which they are able to trade.

Another problem is that the financial sophistication implied by the capacity to implement any desired state-contingent consumption plan through financial transactions is at odds with the core assumption of the model, namely, that agents are willing to make investments based on their own beliefs, even though they are aware that others hold different beliefs. This assumption is, on the face of things, inconsistent with the result of Aumann (1976) that, given common knowledge of rationality, such disagreement is impossible.

In this paper, we examine the Blume-Easley survival result in a context where agents may be constrained to trade in a subset of the assets available in the market as a whole. This constraint may be externally imposed, as in the case of institutions restricted to a limited set of assets, or it may arise from cognitive limitations. In either case, the result is that agents are limited to trades that lie within the span of the set of financial assets available to them.

We begin with an exogenously given state space, so that assets may be described as bundles of state-contingent claims. Similarly to Sandroni (2005), on this state space, we 
consider a (potentially incomplete) set of securities, that span a partition (referred to as the market partition) of this state space. That is, each security pays 1 unit conditional on a given element of the partition being realized and nothing otherwise. To capture differential constraints, we define for each agent a partition, in general coarser than the market one, and assume that each agent has access to a set of assets that span his individual partition. We refer to this partition as the constrained partition available to a given agent. If the partition of each agent coincides with the market partition, the usual case of incomplete markets arises. An agent can trade in a strictly larger set of assets than another agent if the constrained partition of the first agent is strictly finer than that of the second. ${ }^{1}$

First, we consider an economy in which trade occurs only at time 0 . With a set of assets specified as above, the span of the market is a subspace of the space of state-contingent claims. Consumption streams financed by asset trades must be measurable with respect to this subspace. Similarly, each agent's net trades must be measurable with respect to his constrained partition. While we do not impose short sale constraints, all nonnegative consumption streams measurable with respect to the span of the market can be financed without recourse to short selling.

We consider the impact of differential asset constraints, and the associated differences in partition refinement, on allocations and survival in markets. We first construct a simple example of an economy with differential asset constraints and identical beliefs, and show that allocations and prices in general differ from those in the complete markets case. In particular, differential asset constraints might lead (force) less constrained agents to buy only partial insurance against idiosyncratic risk, even though, by trading among themselves, they could fully insure themselves against idiosyncratic risk. We illustrate this in Examples 1 and 2. For Example 1, we provide conditions under which less constrained agents will choose to bear more risk than constrained agents, in return for higher expected consumption.

We next demonstrate that these effects are persistent in that constrained agents are not driven out of the market. In particular, whenever agents have equal discount factors and identical correct beliefs, and the constrained partitions are nested, all agents almost surely survive. Thus, under these conditions, the coarseness of the partition is irrelevant for survival, even though it alters the equilibrium allocation and even though, ceteris paribus, agents with coarser partitions achieve lower welfare in equilibrium. In the special case of incomplete markets, with no differential constraints, the main results of Sandroni (2005) and Blume and Easley (2006) remain valid.

Differential constraints make a difference when agents have heterogeneous beliefs. Provided constraints are relevant (in a sense we will define more precisely), an agent whose beliefs are further away from the truth can survive if the agents with beliefs closer to the truth are more constrained. The key to the result is that agents may survive either because their probability judgements are more accurate than those of others or because they can trade in asset markets from which agents with more accurate judgements are excluded. This result is in stark contrast to the results cited above, which preclude belief heterogeneity in bounded economies with complete markets and expected utility maximization.

\footnotetext{
${ }^{1}$ In Guerdjikova and Quiggin (2019), we use results by Choquet (1956), Kendall (1962), Polyrakis (1999), and Kountzakis and Polyrakis (2006) to demonstrate how this asset structure can be generated from a general set of assets available in the economy and a general set of financial constraints. A sufficient condition called internal completeness is for the set of assets to contain an appropriate set of put and call options so that the implied set of payoffs is a sublattice of the Euclidean space.
} 
In particular, suppose that aggregate risk persists in the limit on those contingencies that can only be traded by one individual. While an individual will always try to smooth consumption across contemporaneous states, the inability to trade with others across two or more states with distinct initial endowments means that he will have strictly positive consumption on at least one of them in the indefinite future and will, therefore, not vanish.

These results are also distinct from those obtained in the context of incomplete markets with sequential trading (Beker and Chattopadhyay (2010), Coury and Sciubba (2012)), where correct beliefs may not guarantee survival. In our model, agents with correct beliefs always survive, regardless of the constraints they face. Agents with incorrect beliefs may also survive provided they face less financial constraints. We consider the case of sequential trading in Appendix A. We show that the resulting survival criterion is much more complex and cannot, in general, be reduced to comparisons of the exogenous characteristics of the agents. Nevertheless, our main insights remain robust to such an extension.

We next consider the case (arguably the most realistic) where the sets of assets different agents have access to are not related with respect to inclusion. We call such financial constraints nonnested. In particular, we look at an economy in which each agent can trade between a pair of states that no other agent can trade on, and we demonstrate that all agents survive a.s. regardless of their beliefs and discount factors. Adding an unconstrained agent with correct beliefs to such an economy implies that the surviving traders, regardless of the constraints they face, must have correct beliefs and equal discount factors.

Next, we study the impact of an individual gaining access to new assets, with a resulting refinement of the constrained partition. This might occur exogenously as a result of a policy change, such as the replacement of a defined benefit pension plan by a defined contribution scheme in which participants are free to choose their own investment strategy. Alternatively the change might arise endogenously, as individuals become aware of assets they had not previously considered or become more confident in their ability to evaluate a larger set of assets. ${ }^{2}$

We show that the agent almost surely vanishes, unless, upon gaining access to the market, he adopts beliefs at least as close to the truth as those of other agents already trading in this market. In particular, if an agent with correct beliefs is present in the economy, a constrained agent who exogenously gains access to new markets and who either has to guess the correct probabilities or use Bayesian updating to learn them will vanish almost surely. More generally, we show that while markets with less constrained agents provide more opportunities for risk sharing, they also pose greater risk for the survival of traders who might misjudge probabilities.

The assumption that agents facing relaxed constraints adopt beliefs close to those of the market is plausible in the case of an agent for whom the relaxation of constraints is the result of increased awareness about investment options. On the other hand, it is less plausible where access to new assets arises exogenously, for example, as a result of changes in regulation.

This finding is also relevant in the consideration of hedge funds, which invest on behalf of high wealth individuals. Hedge fund investors are assumed to be sufficiently sophisticated that they do not require prudential controls on their investment strategies. However, many hedge funds incur losses sufficiently severe that they are terminated. Robust (that is, constrained optimal) investment strategies have been proposed that would reduce the vulnerability of hedge fund portfolios to incorrect beliefs.

\footnotetext{
${ }^{2}$ We thank an anonymous referee for suggesting this interpretation.
} 
Apart from extending the analysis of Blume and Easley (2006) to a market with differential financial constraints, the results derived in this paper are of a broader interest. First, from a normative point of view, our results provide a potential rationale for restrictions on the investment choices available to certain classes of agents. An example is a requirement for trustees to restrict their holdings to investment grade securities such as highly rated bonds.

Second, going beyond the analysis of financial markets, the results derived here are relevant for the rapidly growing body of research on macroeconomic models with heterogeneous agents. A crucial requirement for such models is the existence of a long run equilibrium in which heterogeneous agents survive and are relevant in the determination of state-contingent prices. Our analysis provides such an instance of persistent heterogeneity in beliefs and constraints arising in natural settings.

\section{LITERATURE SURVEY}

\subsection{Survival in Markets}

The idea that markets select for firms and agents that make optimal choices may be traced back to mid-20th century debates about the economics of the firm. Responding to evidence by Hall and Hitch (1939) that firms did not equate marginal cost and marginal revenue in their pricing decisions, Alchian (1950) and Friedman (1953) argued that markets would nonetheless select for those firms that adopted profit maximizing principles. Turning this argument around, Stigler (1958) argued for the "survivor" principle, namely that the efficient scale of operation of firms in a given market could be inferred from the scale of those firms that survived.

Similar ideas emerged in the early 2000s in relation to financial markets. Blume and Easley (2006) and Sandroni (2000) studied the evolution of long-lived optimizing investors with different beliefs and preferences. In bounded economies with complete markets populated by subjective expected utility (SEU) investors, only investors with correct beliefs survive. Risk preferences are irrelevant for survival.

Blume and Easley (2006) and Massari (2013) also develop, in the context of a bounded economy, conditions for survival for agents who are Bayesian learners. In an unbounded economy with a bond, Cogley, Sargent, and Tsyrennikov (2014) show that Bayesian learning need not vanish in the presence of agents who know the truth. In our setting, where the assumption of boundedness is maintained, the result of Blume and Easley (2006) on Bayesian learners vanishing relative to investors with correct beliefs still applies and has some interesting consequences when financial constraints are relaxed.

The situation is more complex in the case of incomplete markets with sequential trading. In this case, correct beliefs are neither necessary nor sufficient for survival (Beker and Chattopadhyay (2010), Coury and Sciubba (2012)). Beker and Chattopadhyay (2010) demonstrate that the dynamics of an economy with incomplete markets is highly nontrivial: in some cases an agent with correct beliefs can vanish; in others the economy might exhibit cycles in which the consumption of each of the agents approaches 0 infinitely often. Coury and Sciubba (2012) show that in incomplete markets, it is always possible to construct an equilibrium in which an agent with incorrect beliefs survives. However, the equilibrium is constructed in such a way that the allocation coincides with an equilibrium in which all agents have correct beliefs. In contrast, in our setting, incorrect beliefs (provided they survive), will have an impact on prices and allocations.

Finally, several recent papers have raised issues with the concept of Pareto optimality in the presence of heterogeneous beliefs (see, for example, Weyl (2007)) and have 
introduced alternative criteria for the optimality of equilibrium allocations such as beliefneutral efficiency in Brunnermeier, Simsek, and Xiong (2014), no-betting Pareto improvement defined by Gilboa, Samuelson, and Schmeidler (2014), and true Pareto efficiency studied by Blume et al. (2018). These approaches argue that certain trades due to speculation (differences in beliefs) have to be scrutinized to determine whether they are truly mutually improving. In this context, constraints imposed on trades might be socially optimal. Posner and Weyl (2013) discuss the practical implementation of financial regulation when traders have different beliefs.

While Blume et al. (2018) look at markets that are incomplete in the same way for all agents, we consider differential financial constraints. Our results show a trade-off between expanding the set of possible trades and survival at the level of an individual. They raise the question of whether investor type-specific financial constraints might serve the double role of maximizing the number of nonspeculative trades while at the same time preventing investors with incorrect beliefs from engaging in speculation leading to bankruptcy.

\subsection{Constrained Investment}

There is extensive evidence to show that the investment choices of households are constrained. Zhou (2015), using data from the Panel Survey on Income Dynamics, estimates the stockmarket participation rate at 50 percent. More generally, as observed by French and Poterba (1991), investors hold less foreign equity and more domestic equity than would be considered optimal on the basis of a standard risk-return trade-off.

Many kinds of constraints in markets have been considered in the financial market literature. Goodhart, Romanidis, Tsomokos, and Shubik (2016) provide an extensive review and discuss the significance of market imperfections in macroeconomic modelling. Borrowing constraints have been introduced in the form of liquidity constraints, that is, when certain types of income cannot be traded upon in advance (see, for example, Detemple and Serrat (2003) and Kiyotaki and Moore (2005)), and in the form of collateral requirements (see Geanakoplos and Zame (2014) and Gottardi and Kubler (2015), as well as the references therein). Kehoe and Levine (2001) compare the two types of models and explain the different implications of such restrictions on the dynamics of asset prices and allocations.

Gottardi and Kubler (2015), in particular, discuss the efficiency of equilibria with collateral requirements and show that, in certain cases, sharper constraints can lead to Pareto improvement. Araujo, Fajardo, and Pascoa (2005) endogenize the choice of collateral and show that such equilibria are constrained Pareto efficient (given the possibility of default). However, in this literature, the possibility of default is not agent-specific. ${ }^{3}$ Furthermore, the efficiency analysis is conducted assuming that all agents have correct beliefs.

Limited participation has been studied in the literature under several different aspects. Balasko, Cass, and Shell (1995) consider a one period economy with two states of the world, in which some of the agents cannot trade across the two states, and they discuss uniqueness of equilibria and the existence of sunspots. Multiple papers show that imposing restrictions on the assets traded can explain some of the stylized facts in financial markets, notably the equity premium puzzle, the foreign equity puzzle, and procyclical price-dividend ratios (see Errunza and Losq $(1985,1989)$, Basac and Cuoco (1998), Guo

\footnotetext{
${ }^{3}$ Whenever the collateral requirements are exogenously fixed, as in the model of Gottardi and Kubler (2015), these are also asset-specific and not agent-specific.
} 
(2004), and Guevenen (2009)). Brav, Constantinides, and Geczy (2002) summarize the empirical evidence.

These papers mostly assume two types of agents with specific utility functions (logarithmic or constant relative risk aversion (CRRA)) and two assets. One of the agents is restricted and can only trade in one of the assets (a bond or his home security), while the second agent is unrestricted and can hold both securities. Both types of agents have correct beliefs. In contrast, we do not restrict the number or the type of traders in our market and consider participation constraints that might concern any number of assets. Furthermore, none of these studies addresses the issue of survival of constrained traders in such markets. Hence, the question of whether such deviations of prices from fundamentals can be persistent is so far unresolved. Finally, the fact that most of the papers consider agents with correct beliefs implies that participation constraints distort the market allocation and, hence, removing them brings the market closer to efficiency (Errunza and Losq (1989)). In contrast, as explained above, when (some) agents have incorrect beliefs, the appropriate notion of efficiency might change and, thus, the impact of relaxing constraints on efficiency is no longer obvious.

While in our model, constraints are taken to be exogenous, a number of studies address also the issue of endogenous limited participation (Allen and Gale (1994), Calvet, Gonzalez-Eiras, and Sodini (2004)). In these models, there is a fixed cost of participation in a given market and agents decide whether to invest in a given market, depending on whether the obtained returns will compensate them for the incurred cost. Calvet, Gonzalez-Eiras, and Sodini (2004) study the impact of financial innovation, and conclude that it allows for better risk sharing and reduces risk premia, thus, bringing prices closer to fundamentals, but that it can also reduce participation in the market. In contrast, Cao, Wang, and Zhang (2005) show that Knightian uncertainty, that is, uncertainty about the process determining dividends, can lead to endogenous participation. A similar idea is used by Easley and O'Hara (2009) to show how participation in the stock market can be affected by exogenous shocks, which increase the amount of uncertainty. Both papers present models of a one period economy and use ambiguity aversion to model uncertainty about the parameters of the distribution of dividends. In contrast, we model an infinite horizon economy with expected utility maximizers. We show that expanding the set of assets available to an agent increases his expected discounted payoff, but also requires the agent to form beliefs over a larger state space and, thus, increases the probability of vanishing from the market.

\section{THE MODEL OF THE ECONOMY}

\subsection{Time and Uncertainty}

Let $\mathbb{N}=\{0 ; 1 ; 2 ; \ldots\}$ denote the set of time periods. Uncertainty is modelled through a sequence of random variables $\left\{\mathcal{S}_{t}\right\}_{t \in \mathbb{N}}$, each of which takes values in a finite set $S$. We set $S_{0}=\left\{s_{0}\right\}$, that is, no information is revealed in period 0 . Denote by $s_{t} \in S$ the realization of random variable $\mathcal{S}_{t}$. Denote by $\Omega=\prod_{t \in \mathbb{N}} S$ the set of all possible observation paths, with representative element $\sigma=\left(s_{0} ; s_{1} ; s_{2} \ldots s_{t} \ldots\right)$. Finally denote by $\Omega_{t}=\prod_{\tau=0}^{t} S$ the collection of all finite paths of length $t$ (ignoring period 0 , which is common to all paths), with representative element $\sigma_{t}=\left(s_{0} ; s_{1} ; s_{2} \ldots s_{t}\right)$. We will write $s\left(\sigma_{t}\right)$ for the state realized on path $\sigma$ in period $t$. Each finite observation path $\sigma_{t}$ identifies a decision/observation node and the set of all possible observation paths $\Omega$ can also be seen as the set of all nodes.

We can represent the information revelation process in this economy through a sequence of finite partitions of the state space $\Omega$. In particular, define the cylinder with base 
on $\sigma_{t} \in \Omega_{t}, t \in \mathbb{N}$, as $Z\left(\sigma_{t}\right)=\left\{\sigma \in \Omega \mid \sigma=\left(\sigma_{t} \ldots\right)\right\}$. Let $\mathbb{F}_{t}=\left\{Z\left(\sigma_{t}\right) \mid \sigma_{t} \in \Omega_{t}\right\}$ be a partition of the set $\Omega$. Clearly, $\mathbb{F}=\left(\mathbb{F}_{0} \ldots \mathbb{F}_{t} \ldots\right)$ denotes a sequence of finite partitions of $\Omega$ such that $\mathbb{F}_{0}=\Omega$ and $\mathbb{F}_{t}$ is finer than $\mathbb{F}_{t-1}$.

Let $\mathcal{F}_{t}$ be the $\sigma$-algebra generated by partition $\mathbb{F}_{t} ; \mathcal{F}_{0}$ is the trivial $\sigma$-algebra. Let $\mathcal{F}$ be the $\sigma$-algebra generated by $\bigcup_{t \in \mathbb{N}} \mathcal{F}_{t}$. It can be shown that $\left\{\mathcal{F}_{t}\right\}_{t \in \mathbb{N}}$ is a filtration.

We define on $(\Omega ; \mathcal{F})$ a probability distribution $\pi$. Intuitively, $\pi$ describes the evolution of the state process in the economy. In what follows, for brevity, we abuse notation slightly by denoting $\pi\left(Z\left(\sigma_{t}\right)\right)=\pi\left(\sigma_{t}\right)=\pi\left(s_{0} ; s_{1} ; s_{2} \ldots s_{t}\right)$. The one-step-ahead probability distribution $\pi\left(s_{t+1} \mid \sigma_{t}\right)$ at node $\sigma_{t}$ is determined by

$$
\pi\left(s_{t+1} \mid \sigma_{t}\right)=\pi\left(s_{0} \ldots s_{t} ; s_{t+1} \mid s_{0} \ldots s_{t}\right)=\frac{\pi\left(s_{0} \ldots s_{t} ; s_{t+1}\right)}{\pi\left(s_{0} \ldots s_{t}\right)} \quad \text { for any } s_{t+1} \in S .
$$

In words, $\pi\left(s_{t+1} \mid \sigma_{t}\right)$ is the probability under distribution $\pi$ that the next observation will be $s_{t+1}$, given that we have reached node $\sigma_{t}$.

We will assume that the true process of the economy is independent and identically distributed (i.i.d.) and write $\pi\left(s_{t+1}=s \mid \sigma_{t}\right)=\pi(s)$. Note that this does not restrict the endowment process to be i.i.d.. The measurability requirements on the endowment process are specified below.

\subsection{Assets and Financial Constraints}

In the classic model of Arrow and Debreu (1954), agents can trade on every possible contingency. In reality, this type of trade usually occurs via asset markets.

Restrictions on the kinds of assets in which an agent can trade imply a coarsening of the state space with respect to which their trades must be measurable. For example, an agent with an initially nonstochastic endowment, who can trade only in bonds and an index fund, can allocate consumption only in ways that are measurable with respect to the partition generated by aggregate consumption.

Another example arises when two agents might have access to the same set of domestic financial markets, but only one of them is willing and able to trade in global markets. The less constrained agent would have access to a state space derived as the Cartesian product of the state of the domestic economy and the state of the world economy, while the more constrained agent would have access to a coarser quotient space, in which all states of the world economy were treated as indistinguishable. In effect, the more constrained agent displays "home bias" as in French and Poterba (1991).

Consider a partition of the state space $S, W^{0}$, with representative element $w$ and the corresponding partition of $\Omega_{t}, \Omega_{t}^{0}$, given by $\Omega_{t}^{0}=\prod_{\tau=1}^{t} W^{0}$ and $\Omega^{0}=\prod_{\tau=1}^{\infty} W^{0}$ with representative elements $\omega_{t}$ and $\omega$, respectively. For a given period $t \geq 1$, we assume that the assets that pay at time $t$ and are available for trade at period $t=0$ are those that pay 1 unit on a given element of the partition $\Omega_{t}$ and nothing otherwise; that is, $\tilde{A}_{t}^{0}=\left\{a_{\omega_{t}}\left(\sigma_{t}\right)=\mathbf{1}_{\omega_{t}}\right\}$. The collection of all such sets $\tilde{A}^{0}=\bigcup_{t} \tilde{A}_{t}^{0}$ represents the set of assets available to the economy as a whole. Hence, markets can be complete (when $W^{0}=\left\{\{s\}_{s \in S}\right\}$ ) or incomplete.

Consider a finite set $I$ of $n$ infinitely lived agents. For each agent, define a partition of the state space $S, W^{i}$, which is at least as coarse as $W^{0}$, and the corresponding partition of $\Omega_{t}, \Omega_{t}^{i}=\prod_{\tau=1}^{t} W^{i}$ and $\Omega^{i}=\prod_{t=1}^{\infty} W^{i}$. This partition represents agent $i$ 's financial constraints in that the assets available to agent $i$ at $t$ are those that pay conditionally on $\Omega_{t}^{i}$. $\tilde{A}_{t}^{i}=\left\{a_{\omega_{t}^{i}}\left(\sigma_{t}\right)=\mathbf{1}_{\omega_{t}^{i}}\right\}$. The set of all assets $i$ has access to is $\tilde{A}^{i}=\bigcup_{t} \tilde{A}_{t}^{i}$. 
REMARK 1: Propositions 1 and 2 in Guerdjikova and Quiggin (2019) give sufficient conditions (internal completeness and intertemporal asset structure) under which a set of assets $A^{0}$ for the economy generates a space of positive payoff streams at time $t \geq 1$ identical to that of $\tilde{A}_{t}^{0}$, for some partitions of $\Omega_{t}, \Omega_{t}^{0}$ and of $S, W^{0}$, such that $\Omega_{t}^{0}=\prod_{\tau=1}^{t} W^{0}$. The realistic scenario, in which the set of assets is smaller than the underlying state space, corresponds to a nontrivial partition $\Omega_{t}^{0}$ that is coarser than $\Omega_{t}$ for all $t$ and, thus, $W^{0}$ that is coarser than $S$. The payoffs of all available assets are measurable with respect to the obtained partition.

Similarly, the agents' partitions can be generated by assuming that each agent $i$ has access to a subset of all available assets $A^{i} \subseteq A^{0}$ satisfying the same properties. The special case of $A^{i}=A^{0}$ and, thus, $W^{i}=W^{0}$ for all agents corresponds to the standard case of incomplete markets.

If $A^{j} \subset A^{i}$ for two agents $i$ and $j$, then $W^{i}$ is a strict refinement of $W^{j}$. In this case, we say that $i$ is less constrained than $j$ or that $i$ has access to a larger set of portfolios than $j$.

Below, we specify the agents' endowments in terms of the assets available to them and, thus, implicitly assume that initial endowments are also measurable with respect to (w.r.t.) the relevant partitions. ${ }^{4}$ Hence, the total endowment of the economy at $t$ will also be measurable with respect to $\Omega_{t}^{0}$. It follows that without loss of generality (w.l.o.g.), we can take the partition $\Omega_{t}^{0}$ to coincide with $\Omega_{t}$. Note, however, that this distinction between $\Omega_{t}^{0}$ and $\Omega_{t}$ becomes relevant if we wish to consider financial innovation.

Finally, we remark that from the point of view of agent $i$, markets are complete with respect to $\Omega^{i}$, that is, the agent can generate any stream of payoffs that is measurable ${ }^{5}$ with respect to $\Omega^{i}$.

Given the so defined individual partitions $\Omega^{i}$, from the point of view of agent $i$, the relevant filtration is generated by finite partitions of the set $\Omega^{i},\left(\mathbb{F}_{t}^{i}\right)_{t \in \mathbb{N}}$, defined analogously to $\left(\mathbb{F}_{t}\right)_{t \in \mathbb{N}}$. Note that for each $t, \mathbb{F}_{t}^{i}$ is coarser than the corresponding $\mathbb{F}_{t}$. We will denote by $\mathcal{F}_{t}^{i}$ the $\sigma$-algebra generated by partition $\mathbb{F}_{t}^{i} ; \mathcal{F}_{0}^{i}=\mathcal{F}_{0}$ is the trivial $\sigma$-algebra. Let $\mathcal{F}^{i}$ be the $\sigma$-algebra generated by $\bigcup_{t \in \mathbb{N}} \mathcal{F}_{t}^{i}$. Just as above, $\left\{\mathcal{F}_{t}^{i}\right\}_{t \in \mathbb{N}}$ is a filtration. Note that for each $\sigma_{t}$ and the corresponding state realized at time $t, s\left(\sigma_{t}\right)$, there is an element of the partition $w^{i}$ with $s\left(\sigma_{t}\right) \in w^{i}$. We will denote the element of the partition of agent $i$ realized at time $t$ on path $\sigma$ by $w^{i}\left(\sigma_{t}\right)$.

Agent $i$ 's beliefs $\pi^{i}$ are defined on $\left(\Omega^{i} ; \mathcal{F}^{i}\right)$. The one-step ahead probability distribution $\pi^{i}\left(w_{t+1}^{i} \mid \omega_{t}^{i}\right)$ is defined analogously to $\pi\left(s_{t+1} \mid \sigma_{t}\right)$. Obviously, $\mathcal{F}$ is finer than $\mathcal{F}^{i}$ and, hence, the true probability distribution $\pi$ on $(\Omega ; \mathcal{F})$ specifies a probability distribution on $\left(\Omega^{i} ; \mathcal{F}^{i}\right)$ with $\pi\left(\omega_{t}^{i}\right)=\pi\left\{\sigma_{t} \mid s_{\tau} \in w_{\tau}^{i}\right.$ for all $\left.\tau \in\{1 \ldots t\}\right\}$. We will say that $i$ 's beliefs are correct if they coincide with the restriction of $\pi$ to $\left(\Omega^{i} ; \mathcal{F}^{i}\right)$.

For most of the paper, we will restrict attention to beliefs that describe an i.i.d. process, $\pi^{i}\left(w_{t+1}^{i}=w^{i} \mid \omega_{t}^{i}\right)=\pi^{i}\left(w^{i}\right)$.

There is a single good consumed in positive quantities. Each agent $i$ is endowed at $t=0$ with some of the consumption good, $e^{i}\left(\sigma_{0}\right)$, and with a portfolio $\bar{\theta}^{i}$ of securities in

\footnotetext{
${ }^{4}$ In particular, for an agent who has access only to a bond, initial endowment is nonstochastic (though it can depend on the time period). Nontradable idiosyncratic risk can be captured by endowing an agent with risky assets, which are nontradable, that is, which are only available to this particular agent and no one else in the economy; see Example 3 below. The impact of such nontradable idiosyncratic risk on survival is considered in Propositions 7 and 8 .

${ }^{5}$ Furthermore, any nonnegative consumption stream can be obtained without recurring to short sales. Thus, even though the financial constraints we impose in the paper do not restrict short selling, the equilibrium allocation does not include short sales.
} 
$\tilde{A}^{i}$, which pay in terms of the consumption good $\bar{\theta}^{i}: \Omega \backslash \sigma_{0} \rightarrow \prod_{t \in \mathbb{N}} \mathbb{R}_{+}$. The payoff of $i$ 's portfolio is measurable with respect to $\left(\Omega^{i} ; \mathcal{F}^{i}\right)$ and, hence, his initial endowment stream is given by a function $e^{i}: \Omega \rightarrow \prod_{t \in \mathbb{N}} \mathbb{R}_{+}$. This stream is also measurable with respect to $\left(\Omega^{i} ; \mathcal{F}^{i}\right)$. The initial endowment at node $\omega_{t}^{i}$ coincides with the number of generalized unit securities in the initial portfolio that pay conditional on $\omega_{t}^{i}$, that is, $e^{i}\left(\omega_{t}^{i}\right)=\bar{\theta}^{i}\left(a_{\omega_{t}^{i}}\right)$. The total endowment of the economy is denoted by $e=\sum_{i} e^{i}$.

Since agent $i$ can only trade in assets in $\tilde{A}^{i}$, agent $i$ 's consumption set consists of functions $c^{i}: \Omega \rightarrow \prod_{t \in \mathbb{N}} \mathbb{R}_{+}$measurable with respect to $\left(\Omega^{i} ; \mathcal{F}^{i}\right)$.

Agents are assumed to be expected utility maximizers given their trading constraints and their (subjective) beliefs. ${ }^{6}$ Agent $i$ 's utility function for risk is denoted by $u_{i}$ and his discount factor is $\beta^{i}$.

We will impose the following assumptions on utility functions and endowments, which are standard in the survival literature:

ASSUMPTION 1: All agents are expected utility maximizers with utility functions for risk $u_{i}$ : $\mathbb{R}_{+} \rightarrow \mathbb{R}$ that are twice continuously differentiable, strictly concave, and satisfy $\lim _{c \rightarrow 0} u_{i}^{\prime}(c)=$ $\infty$ and $\lim _{c \rightarrow \infty} u_{i}^{\prime}(c)=0$.

Assumption 2: Individual endowments are strictly positive, $e^{i}\left(\sigma_{t}\right)>0$ for all $i$ and $\sigma_{t}$. Aggregate endowments are uniformly bounded away from zero and uniformly bounded from above. Formally, there is an $m>0$ such that $\sum_{i \in I} e^{i}\left(\sigma_{t}\right)>m$ for all $i, \sigma_{t}$; moreover, there is an $m^{\prime}>m>0$ such that $\sum_{i \in I} e^{i}\left(\sigma_{t}\right)<m^{\prime}$ for all $\sigma_{t}$.

ASSUMPTION 3: For all $s \in S, \pi(s)>0$, and for all $i \in I, \pi^{i}\left(w^{i}\right)>0$ for all $w^{i} \in W^{i}$.

Assumption 1 implies that the agent would never choose zero consumption in a state he believes to have a positive probability. Assumption 2 ensures that endowments are uniformly bounded away from 0 and above. Given the i.i.d. structure imposed on the true process and on beliefs, Assumption 3 states that one-step-ahead probabilities of all states of the world are positive and that all subjective beliefs assign a positive one-stepahead probability to every element in their respective partitions. In particular, there is a $\underline{\pi}>0$ such that $\pi(s)>\underline{\pi}$ and $\pi^{i}\left(w^{i}\right)>\underline{\pi}$ for all $i \in I$ and all $w^{i} \in W^{i}$. This assumption is analogous to imposing absolute continuity of one-step-ahead subjective beliefs with respect to the true one-step-ahead probabilities (as in Blume and Easley (2006)). Taken together, Assumptions 1 and 3 ensure that no agent vanishes in finite time.

In economies with bounded endowments and complete markets, and populated by expected utility maximizers, only beliefs and discount factors matter for survival. In particular, if all agents are equally patient, agents with incorrect beliefs vanish a.s. in the presence of agents with correct beliefs. In contrast, in unbounded economies, risk attitudes also matter for survival, and agents with incorrect beliefs can survive. So as to disentangle the effects of asset constraints on survival from those of risk attitude, we restrict our attention to the case of bounded economies.

\section{EQUILIBRIUM IN MARKETS WITH DIFFERENTIAL FINANCIAL CONSTRAINTS}

Our main results are derived on the assumption that agents trade their portfolios at time 0 with no subsequent opportunity for retrading. Thus, the approach taken in the

\footnotetext{
${ }^{6} \mathrm{An}$ expected utility representation with a coarse subjective state space has been recently axiomatized by Minardi and Savochkin (2016).
} 
main part of the paper mimics that of Sandroni (2005), in which there is a single period of trade, but information is subsequently revealed according to the structure presented in Section 3. This assumption greatly simplifies the analysis and allows us to derive a simple criterion for survival in economies with differential constraints. Differently from Sandroni, we allow consumption to occur in time. In Appendix A, we extend the analysis to the case of sequential trading. Even though the definition and the analysis of the equilibrium are substantially different for the two cases, we show that the main insights of the paper are robust to such a modification.

DEFINITION 1: An equilibrium of the economy with differential financial constraints consists of an integrable ${ }^{7}$ price system $\left(p\left(\sigma_{t}\right)\right)_{\sigma_{t} \in \Omega}$ and a consumption stream $c^{i}$ for every agent $i$ such that (i) all agents $i \in\{1 \ldots n\}$ are maximizing their expected utility given the price system subject to choosing consumption streams that are measurable relative to their constrained partition, and (ii) markets clear:

$$
\begin{gathered}
c^{i}=\arg \max _{c^{i}} V_{0}^{i}\left(c^{i}\right) \\
=\arg \max _{c^{i}}\left\{u_{i}\left(c^{i}\left(\sigma_{0}\right)\right)+\sum_{t=1}^{\infty} \beta_{i}^{t} \sum_{\omega_{t}^{i} \in \Omega_{t}^{i}} \pi^{i}\left(\omega_{t}^{i}\right) u_{i}\left(c^{i}\left(\omega_{t}^{i}\right)\right)\right. \\
\text { s.t. } \sum_{t \in \mathbb{N}} \sum_{\omega_{t}^{i} \in \Omega_{t}^{i}} \sum_{\sigma_{t} \in \omega_{t}^{i}} p\left(\sigma_{t}\right) c^{i}\left(\omega_{t}^{i}\right) \\
\left.\leq \sum_{t \in \mathbb{N}} \sum_{\omega_{t}^{i} \in \Omega_{t}^{i} \sigma_{t} \in \omega_{t}^{i}} p\left(\sigma_{t}\right) e^{i}\left(\omega_{t}^{i}\right)\right\}, \\
\sum_{i \in I} c^{i}\left(\sigma_{t}\right)=\sum_{i \in I} e^{i}\left(\sigma_{t}\right) \quad \forall \sigma_{t} \in \Omega .
\end{gathered}
$$

An equilibrium in an economy with differential financial constraints is consistent with the fact that different agents face different financial constraints, trade on different partitions of the state space, and, hence, effectively optimize over different sets of commodities (consumption on events $\omega_{t}^{i}$ rather than $\sigma_{t}$ ). The equilibrium can be interpreted in the following way: in period 0 , before any uncertainty is resolved, all agents sell their initial endowment to an intermediary ${ }^{8}$ at market prices and use the revenues to buy their preferred consumption paths $c^{i}$ for all future contingencies on which they can trade. The price of

\footnotetext{
${ }^{7}$ Integrability of $\left(p\left(\sigma_{t}\right)\right)_{\sigma_{t} \in \Omega}$ on $(\Omega ; \mathcal{F} ; \mu)$, where $\mu$ is the counting measure or, equivalently, the requirement that the price system is $L^{1}$ on $(\Omega ; \mathcal{F} ; \mu)$, ensures that the total wealth of an individual investor is finite, that is, that the sum $\sum_{t \in \mathbb{N}} \sum_{\omega_{t}^{i} \in \Omega_{t}^{i}} \sum_{\sigma_{t} \in \omega_{t}^{i}} p\left(\sigma_{t}\right) e^{i}\left(\omega_{t}^{i}\right)$ is well defined; see Bewley (1972, p. 516).

${ }^{8}$ The fact that agents can trade through an intermediary means that the restriction of measurability is imposed only on the total net trades of a given agent. One could alternatively define an equilibrium through bilateral trades and require that the bilateral net trades be measurable with respect to each agent's partition. This will, in general, restrict the set of potential equilibrium allocations. Note, however, that when agents' partitions are nested, the agent with the finest partition can de facto play the role of an intermediary and, thus, measurable bilateral net trades supporting the equilibrium allocation always exist. The same is true for an economy with two agents with nonnested partitions. More generally, as pointed out to us a by a referee, the two equilibrium notions will not coincide (e.g., Remark 2 below need not hold when measurability of bilateral trades is required), and this might have an impact on the existence and the properties of the equilibrium as well as on survival results. Note, however, that in the cases studied below, notably nested partitions, or an economy
} 
consumption contingent on a coarse contingency $\omega_{t}^{i}$ is simply the sum of consumption prices over all nodes $\sigma_{t} \in \omega_{t}^{i}$, that is, $\sum_{\sigma_{t} \in \omega_{t}^{i}} p\left(\sigma_{t}\right)$.

PROPOSITION 1: Under Assumptions 1-3, an equilibrium of the economy with differential financial constraints exists. Furthermore, the equilibrium satisfies, for each $i \in I$ and at each $\omega_{t}^{i}, \omega_{t+1}^{i} \in \Omega^{i}$ such that $\pi\left(\omega_{t+1}^{i}\right)>0$,

$$
\frac{u_{i}^{\prime}\left(c^{i}\left(\omega_{t}^{i}\right)\right)}{\beta_{i} \pi^{i}\left(\omega_{t+1}^{i} \mid \omega_{t}^{i}\right) u_{i}^{\prime}\left(c^{i}\left(\omega_{t+1}^{i}\right)\right)}=\frac{p\left(\omega_{t}^{i}\right)}{p\left(\omega_{t+1}^{i}\right)}=\frac{\sum_{\sigma_{t} \in \omega_{t}^{i}} p\left(\sigma_{t}\right)}{\sum_{\sigma_{t+1} \in \omega_{t+1}^{i}} p\left(\sigma_{t+1}\right)},
$$

where $p(\cdot)$ is the equilibrium price system.

We now provide a simple example to illustrate the impact of differential financial constraints on equilibrium prices and allocations.

EXAMPLE 1: Consider an economy with two agents, Ann and Bob. Their initial endowments in each period depend on whether each of them is employed or not. The assets in the economy are a bond, unemployment insurance claims for Ann and Bob, that is, assets $A$ and $B$, which yield a payment of 1 if the associated agent is unemployed, and an insurance claim against a high unemployment rate in the economy as a whole, which pays 1 when both agents are unemployed and nothing otherwise. The induced state space for an unconstrained agent has four states: $S=\left\{s_{1} \ldots s_{4}\right\}$. In $s_{1}, A$ is employed and $B$ is not; in $s_{2}, B$ is employed, but $A$ is not. In $s_{3}$, both agents are unemployed, and in $s_{4}$, both are employed. Intuitively, states $s_{1}$ and $s_{2}$ can be interpreted as "business as usual," in which unemployment is a matter of idiosyncratic risk, whereas in states $s_{3}$ and $s_{4}$, the economy is subject to aggregate risk (low or high unemployment rates). The initial endowment of an agent is 1 in a state in which he is unemployed and 2 in a state in which he is employed, see Table I.

Assume now that while $A$ has access to all four assets, $B$ can only trade the bond and his own unemployment insurance asset. This induces the partition

$$
W^{B}=\left\{w_{1}^{B}=\left\{s_{1} ; s_{3}\right\} ; w_{2}^{B}=\left\{s_{2} ; s_{4}\right\}\right\} .
$$

TABLE I

INITIAL ENDOWMENTS FOR EXAMPLE 1

\begin{tabular}{lllll}
\hline \hline Initial endowment & $s_{1}$ & $s_{2}$ & $s_{3}$ & $s_{4}$ \\
\hline Ann & 2 & 1 & 1 & 2 \\
Bob & 1 & 2 & 1 & 2 \\
\hline
\end{tabular}

with an unconstrained agent (as in Proposition 8), the results on survival will not depend on the definition chosen. The result of Proposition 7 is based on the fact that trading constraints prevents agents from selling parts of their endowment and, thus, from vanishing. Imposing further constraints on trades will not invalidate this argument. 
Bob's initial endowment respects the measurability assumption imposed above, that is, $e_{s_{1}}^{B}=e_{s_{3}}^{B}=1$ and $e_{s_{4}}^{B}=e_{s_{4}}^{B}=2$.

Consider first the case in which both $A$ and $B$ are unconstrained. Assuming that both have identical (correct) beliefs $\pi$ about the realization of the four states and strictly concave von Neumann-Morgenstern utility functions $u_{A}$ and $u_{B}$, the equilibrium of this economy would fully insure both agents against the idiosyncratic risk, that is, $c^{A}\left(s_{1}\right)=c^{A}\left(s_{2}\right)$ and $c^{B}\left(s_{1}\right)=c^{B}\left(s_{2}\right)$, and, hence, $\frac{p_{1}^{*}}{\pi\left(s_{1}\right)}=\frac{p_{2}^{*}}{\pi\left(s_{2}\right)}$ obtains. As for the allocation across states $s_{3}$ and $s_{4}$, we know that the less risk averse agent will partially insure the more risk averse agent against the aggregate risk. If both agents have identical utility functions, no trade across these two states will occur.

Now consider the situation in which $B$ is constrained and has access only to the two assets that generate the partition $W^{B}$ specified above. The equilibrium allocation described above is no longer feasible, since it specifies $c^{B}\left(s_{1}\right)>1=c^{B}\left(s_{3}\right)$ and would, thus, require $B$ to trade on states $s_{1}$ and $s_{3}$. So what can we say about the equilibrium with financial constraints? First, we can show (see the proof of Claim 1 in Appendix B) that when $B$ is constrained, neither $A$ nor $B$ is insured against idiosyncratic risk in equilibrium. Second, since $u$ is concave, in equilibrium, $1<c^{B}\left(s_{1}\right)=c^{B}\left(s_{3}\right)<c^{B}\left(s_{2}\right)=c^{B}\left(s_{4}\right)<2$, that is, $B$ buys partial insurance against aggregate risk. This in turn implies that state prices are biased relative to the case of no financial constraints: $\frac{p_{1}^{*}}{\pi\left(s_{1}\right)}<\frac{p_{2}^{*}}{\pi\left(s_{2}\right)}$. Finally, if

$$
\pi\left(s_{1}\right) \pi\left(s_{2}\right)-\pi\left(s_{3}\right) \pi\left(s_{4}\right) \leq 0,
$$

$A$ 's expected consumption is higher than her expected initial endowment (see the proof of Claim 2 in Appendix B).

The sufficient condition (3) for $A$ to bear more risk and, thus, obtain a higher expected consumption than under her initial endowment will hold if all four states are equally likely. Alternatively, suppose that the state $s_{4}$ has a probability $\pi_{4}>\frac{1}{2}$, that is, full employment is the default state of the economy. Assume also that the two states with idiosyncratic risk, $s_{1}$ and $s_{2}$, are equally probable; $\pi_{1}=\pi_{2}$, that is, the probability that each one of the agents loses his/her job is the same. In this scenario, condition (3) is satisfied as well.

While the example is formulated as a static one, we may show that, assuming equal discount factors, identical von Neumann-Morgenstern functions $u_{A}$ and $u_{B}$, and an initial endowment i.i.d. over time, the static equilibrium will be replicated in every period $t$.

EXAMPLE 2: We now add (to Ann and Bob) two agents Clara and David. Assume that $C$ has the same initial endowment as $A$ and that $D$ has the same initial endowment as $B$; that is, $e^{C}=e^{A}$ and $e^{D}=e^{B}$, see Table II. However, we now assume that Clara has access only to the bond and to Ann's claim for insurance against unemployment (which insures her against her own unemployment), whereas David is unconstrained. Hence, the

TABLE II

INITIAL ENDOWMENTS FOR EXAMPLE 2

\begin{tabular}{lcccc}
\hline \hline Initial endowment & $s_{1}$ & $s_{2}$ & $s_{3}$ & $s_{4}$ \\
\hline Clara & 2 & 1 & 1 & 2 \\
David & 1 & 2 & 1 & 2 \\
\hline
\end{tabular}


partition induced by Clara's financial constraints is given by

$$
W^{C}=\left\{w_{1}^{C}=\left\{s_{1} ; s_{4}\right\}, w_{2}^{C}=\left\{s_{2} ; s_{3}\right\}\right\} .
$$

Assume that all agents have identical correct beliefs on the partitions on which they can trade and that they are all risk averse.

Consider first a (sub)economy consisting of only $B$ and $C$. Given their financial constraints, the idiosyncratic labor income of these two agents is nontradable. ${ }^{9}$ From $B$ 's point of view the only consumption allocations that he prefers to his initial endowment, and that can be derived through trade, are of the type

$$
\left(c^{B}\left(s_{1}\right) ; c^{B}\left(s_{2}\right) ; c^{B}\left(s_{3}\right) ; c^{B}\left(s_{4}\right)\right)=(1+a ; 2-b ; 1+a ; 2-b)
$$

with $a>0, b>0$. However, market clearing implies that the resulting consumption bundle for $C$ specifies

$$
c^{C}\left(s_{1}\right)=2-a \neq c^{C}\left(s_{4}\right)=2+b
$$

and is, thus, inconsistent with her constrained partition. Hence, even though in principle Bob can and might find it optimal to purchase insurance against his own unemployment risk, the fact that Clara is not able to trade in such an asset effectively prevents him from doing so. We examine the impact of such nonnested financial constraints on survival in Propositions 7 and 8.

In contrast, if $A$ and $D$ were the only agents in the economy, they would fully insure each other across states $s_{1}$ and $s_{2}$ in equilibrium: $c^{A}\left(s_{1}\right)=c^{A}\left(s_{2}\right)$ and $c^{D}\left(s_{1}\right)=c^{D}\left(s_{2}\right)$. This result holds independently of whether their utility functions are identical or not. ${ }^{10}$ If their utility functions are identical, no trade on the states with aggregate risk, $s_{3}$ and $s_{4}$, occurs between them.

When all four agents are present in the economy, the equilibrium allocation is different. Suppose, for simplicity. that everyone's beliefs are correct and assign a probability of $\frac{1}{4}$ to each of the states. First, it is impossible to insure everybody against idiosyncratic risk in equilibrium, (see the proof of Claim 3 in Appendix B). Second, in general, the presence of constrained traders in the market ( $B$ and $C$ ) implies that the unconstrained traders $A$ and $D$ cannot be fully insured against idiosyncratic risk either (see the proof of Claim 4 in Appendix B).

Our example demonstrates that markets with differentially constrained agents exhibit different properties from markets in which the same set of assets is available to all agents. First, in such markets, some of the risk sharing opportunities cannot be used, due to the constraints on some agents. Such constraints can affect even trades among unconstrained agents, who, in the absence of constrained agents, would have obtained full insurance against idiosyncratic risk. Second, unconstrained agents might provide additional insurance against aggregate risk to constrained agents, even when both types have identical beliefs and identical risk preferences. Third, in the presence of constrained agents, state prices might be biased. Finally, unconstrained agents might obtain higher expected returns than constrained agents.

\footnotetext{
${ }^{9} \mathrm{We}$ are grateful to an anonymous referee for suggesting this interpretation.

${ }^{10}$ In the presence of differential bargaining power, which might arise from different risk attitudes, the party with less bargaining power might be required to make a state-independent payment so as to reach agreement on the full insurance bargain.
} 


\section{SURVIVAL IN ECONOMIES WITH DIFFERENTIAL FINANCIAL CONSTRAINTS}

In the previous sections, we showed that differential financial constraints can have an impact on equilibrium prices and allocations. This raises the question of whether the impact of constrained agents on prices and allocations is temporary or permanent. Is it the case that their consumption converges to 0 over time, thus driving the equilibrium allocation to that which would have obtained had all agents been unconstrained? In this section, we will show that constrained agents can have a long-term impact on prices and risk sharing.

We define survival as usual.

DEFINITION 2: Agent $i$ vanishes on a path $\sigma$ if $\lim _{t \rightarrow \infty} c^{i}\left(\sigma_{t}\right)=0$. Agent $i$ survives on $\sigma$ if $\lim _{t \rightarrow \infty} \sup c^{i}\left(\sigma_{t}\right)>0$.

In this section, we will assume that Assumptions 1-3 hold, without explicitly stating them in each of the propositions below. We first remark that in the absence of aggregate risk, constraints have no effect on survival.

REMARK 2: In an economy with no aggregate uncertainty, equal discount factors, and identical correct beliefs, all agents will be fully insured. Indeed, since a full insurance consumption stream is measurable w.r.t. any individual partition, the individual financial constraints in such an economy are not binding. Hence, all agents will survive regardless of their individual financial constraints. In this case, the first-order conditions (2) (with correct beliefs) and the equilibrium allocation coincide with those in an unconstrained economy.

Our first result generalizes the main result of Blume and Easley (2006) to apply to agents with access to an identical set of assets and, thus, with identical partitions. It also extends the result of Sandroni (2005) to the case of agents who consume over time. When beliefs are identical, the agents with the highest discount factors can survive. For identical discount factors, only those agents with beliefs closest to the truth can survive.

Proposition 2: Consider two agents $i$ and $j$, who have access to the same set of assets, $A^{i}=A^{j}$, and, thus, have identical partitions, $\Omega^{i}=\Omega^{j}$. If agent's $i$ survival index $\ln \beta_{i}-$ $\sum_{w^{i} \in W^{i}} \pi\left(w^{i}\right) \ln \frac{\pi\left(w^{i}\right)}{\pi^{i}\left(w^{i}\right)}$ is strictly higher than that of $j, j$ vanishes a.s.

Our next result concerns agents who have access to sets of assets $A^{i}$ ordered with respect to inclusion and, hence, nested partitions. It shows that the fact that some agents have access to smaller sets of portfolios alone does not affect survival.

PROPOSITION 3: Consider a population of agents who have sets of available assets ordered with respect to inclusion and, thus, have nested partitions, equal discount factors, and correct beliefs. All agents survive a.s.

Our result shows that whenever agents have equal discount factors and correct beliefs relative to the partition generated by the set of assets they have access to, and the sets of assets are ordered with respect to inclusion, whether the agent has access to a larger or smaller set of assets is irrelevant for survival. In fact, all agents survive. We can relate this result to Example 1. Recall that in a one period economy with Ann unconstrained 
and Bob constrained, insurance against idiosyncratic risk did not obtain in equilibrium. In contrast, the unconstrained agent insured the constrained agent against some of the aggregate risk. The result above implies that these features of the economy will persist in the long run as long as both agents have correct beliefs and equal discount factors.

While agents with access to a smaller set of assets survive, the financial constraints will, in general, (weakly) reduce their welfare.

PROPOSITION 4: Consider an economy with differential financial constraints and assume that agent $i$ is more constrained than $j, A^{i} \subset A^{j}$, and $\Omega^{i}$ is coarser than $\Omega^{j}$. Suppose that the two agents have identical endowments, utility functions, and discount factors $\beta$, and identical beliefs $\pi$ restricted to $\Omega^{i}$. In any equilibrium of the economy with equilibrium allocation $c$, $V_{0}^{j}\left(c^{j}\right) \geq V_{0}^{i}\left(c^{i}\right)$.

Ceteris paribus, an agent who is more constrained will be able to invest conditional on a coarser partition and will, as a result, obtain a lower welfare in equilibrium. Intuitively, the less constrained agent has access to a larger set of trades that he can engage in and will obtain a higher utility from consumption. Note, however, that the weak inequality cannot be replaced by a strict one. For example, if $i$ and $j$ are the only agents in the population, no trade will occur in equilibrium and their welfare will be identical.

We next examine the impact of heterogeneity in discount factors and beliefs on survival when agents' sets of assets are ordered with respect to inclusion. We first show that a more constrained agent $i$ can only survive if his survival index is at least as large as that of a less constrained agent $j$. Thus, either $i$ 's discount factor must be at least as high or his beliefs must be at least as close to the truth as those of $j$.

PROPOSITION 5: If agent $i$ is more constrained than agent $j\left(A^{i} \subset A^{j}\right.$ and $\Omega^{i}$ coarser than $\left.\Omega^{j}\right)$ and $i$ has a strictly lower survival index,

$$
\ln \frac{\beta_{j}}{\beta_{i}}+\left(\sum_{w^{i} \in W^{i}} \pi\left(w^{i}\right) \ln \frac{\pi\left(w^{i}\right)}{\pi^{i}\left(w^{i}\right)}-\sum_{w^{i} \in W^{i}} \pi\left(w^{i}\right) \ln \frac{\pi\left(w^{i}\right)}{\pi^{j}\left(w^{i}\right)}\right)>0,
$$

$i$ vanishes a.s.

Hence, more constrained agents can survive only if they have larger survival indices than less constrained agents. To formulate results for economies in which this conditions is satisfied, we will have to understand when constraints matter in the long term. We will use the following definition.

DEFINITION 3: The financial constraints of agent $i$, given by the partition $\Omega^{i}$, are $i r$ relevant in the limit if for any $\omega^{i} \in \Omega^{i}$ and any $\sigma, \sigma^{\prime} \in \omega^{i}, \lim _{t \rightarrow \infty} e\left(\sigma_{t}\right)-e\left(\sigma_{t}^{\prime}\right)=0$. The financial constraints of agent $i$, given by the partition $\Omega^{i}$, are relevant in the limit if for some $w^{i} \in W^{i}, s$ and $s^{\prime} \in w^{i}$, there is an $\epsilon>0$ such that for any $\sigma, \sigma^{\prime} \in \omega^{i}$,

$$
\lim _{t \rightarrow \infty} \sup \left[e\left(\sigma_{t} ; s\right)-e\left(\sigma_{t}^{\prime} ; s^{\prime}\right)\right]>\epsilon .
$$

The financial constraints of agent $i$ are considered irrelevant if, in the limit, the total endowment of the economy is measurable with respect to agent $i$ 's partition. Such an agent can effectively trade on the total endowment process of the economy in the limit. In contrast, agent $i$ 's constraints are relevant even in the limit if there are at least two 
states that $i$ cannot trade on and in which the total endowment of the economy remains distinct.

Note that if $i$ 's constraints are irrelevant in the limit, then so are those of any agent $j$ who is less constrained, $A^{i} \subset A^{j}$. Similarly if $i$ 's constraints are relevant in the limit, then so are those of a more constrained agent $j$ with $A^{j} \subset A^{i}$.

Consider agent $j$ and for any $\omega^{j} \in \Omega^{j}$ with $\omega^{i} \subseteq \omega^{j}$, define the set $\hat{\Omega}_{t}^{i}\left(\omega_{t}^{j}\right)=\left\{\omega_{t}^{i} \in \Omega_{t}^{i} \mid\right.$ $\omega_{t}^{i} \subseteq \omega_{t}^{j}$ s.t. $\left.\min _{\sigma_{t} \in \omega_{t}^{j}} e\left(\sigma_{t}\right)=\min _{\sigma_{t} \in \omega_{t}^{i}} e\left(\sigma_{t}\right)\right\}$, the set of $\omega_{t}^{i}$ on which the initial endowment of the economy obtains its minimum w.r.t. the set $\omega_{t}^{j}$. Let $\check{\Omega}_{t}^{i}\left(\omega_{t}^{j}\right)=\left\{\omega_{t}^{i} \subseteq \omega_{t}^{j}\right\} \backslash \hat{\Omega}_{t}^{i}\left(\omega_{t}^{j}\right)$.

DEFINITION 4: Let the set of assets available to agents $i$ and $j$ satisfy $A^{i} \supset A^{j}$. The financial constraints of agent $j$ given by the partition $\Omega^{j}$ are irrelevant in the limit w.r.t. those of agent $i$ given by partition $\Omega^{i}$ if for any $\omega^{i} \in \Omega^{i}$ and $\omega^{j} \in \Omega^{j}$ such that $\omega^{i} \subseteq \omega^{j}$, $\lim _{t \rightarrow \infty} \check{\Omega}_{t}^{i}\left(\omega_{t}^{j}\right)=\emptyset$. The financial constraints of agent $j$ are relevant in the limit w.r.t. those of agent $i$ if there is an $\epsilon>0, w^{i} \in W^{i}$ and $w^{j} \in W^{j}, w^{i} \subseteq w^{j}$ such that for any $\omega^{j} \in \Omega^{j}$ and every $\omega^{i} \subseteq \omega^{j}, \omega^{i} \in \Omega^{i}$, (i) $\min _{\left(\sigma_{t^{k}} ; s\right) \in\left(\omega_{t^{k}}{ }^{k} ; w^{i}\right)} e\left(\sigma_{t^{k}} ; s\right)-\min _{\left(\sigma_{t^{k}} ; s\right) \in\left(\omega_{t^{k}}{ }^{k} ; w^{j}\right)} e\left(\sigma_{t^{k}} ; s\right)>\epsilon$ occurs on an infinite set of periods $\left(t^{k}\right)_{k}$ such that (ii) $\min _{\left\{\sigma_{t^{k}+1} \in \omega_{t^{k}+1}^{i} \mid \omega_{t^{k}+1}^{i} \in \check{\Omega}_{t^{k}+1}^{i}\left(\omega_{t^{k}}^{j} ; \omega^{j}\right)\right\}} e\left(\sigma_{t^{k}+1}\right)-$ $\min _{\left(\sigma_{t^{k}} ; s\right) \in\left(\omega_{t^{k}}^{j} ; w^{j}\right)} e\left(\sigma_{t^{k}} ; s\right)>\epsilon$ for all $t^{k}$.

To understand the definition, note that, in general, the initial endowment of the economy is not measurable w.r.t. to $\Omega^{i}$ or $\Omega^{j}$. The maximum consumption of $j$ at $\omega_{t}^{j}$ given the initial endowment of the economy is $\min _{\sigma_{t} \in \omega_{t}^{j}} e\left(\sigma_{t}\right)$, whereas the maximum consumption of $i$ at $\omega_{t}^{i} \subseteq \omega_{t}^{i}$ is $\min _{\sigma_{t} \in \omega_{t}^{i}} e\left(\sigma_{t}\right)$. Furthermore, if $\check{\Omega}_{t}^{i}\left(\omega_{t}^{j}\right)=\emptyset$, then these two values coincide for all $\omega_{t}^{i} \subseteq \omega_{t}^{j}$ : even though $j$ 's partition is coarser, he is no more constrained than $i$ in terms of his maximal possible consumption on $\omega_{t}^{j}$. If this property obtains in the limit, $j$ 's constraints are irrelevant in the limit w.r.t. those of $i$. If, in contrast, $\check{\Omega}_{t}^{i}\left(\omega_{t}^{j}\right) \neq \emptyset$, then $i$ can obtain a strictly higher consumption on $\omega_{t}^{i}$ than $j$ on $\omega_{t}^{j}$, that is, $j$ 's constraint is "relevant" w.r.t. that of $i$. The condition for $j$ 's constraints to be relevant w.r.t. those of $i$ in the limit requires that (i) on every path $\omega^{i} \subseteq \omega^{j}$ on which $w^{i}$ occurs infinitely often (i.o.), $i$ 's maximal consumption exceeds that of $j$ 's by $\epsilon$ i.o., and (ii) on every path $\omega^{j}$ on which $w^{j}$ occurs i.o., the minimal nonzero difference in maximal consumption between $i$ and $j$ on $\omega^{j}$ exceeds $\epsilon$ i.o.

In the sequel, to simplify the discussion, we will concentrate mainly on the case of agents whose constraints are relevant in the limit w.r.t. those of other agents. We will comment briefly on how the results change if agents with constraints that are irrelevant in the limit are introduced.

PROPOSITION 6: Consider a population of agents with available sets of assets ordered with respect to inclusion $A^{1} \supset A^{2} \cdots \supset A^{n}$ and, thus, nested partitions $\Omega^{1}$ strictly finer than $\Omega^{2} \ldots$ strictly finer than $\Omega^{n}$, and ordered survival indices such that for all $i<j$, either

$$
\ln \beta_{i}-\sum_{w^{j}} \pi\left(w^{j}\right) \ln \frac{\pi\left(w^{j}\right)}{\pi^{i}\left(w^{j}\right)}<\ln \beta_{j}-\sum_{w^{j}} \pi\left(w^{j}\right) \ln \frac{\pi\left(w^{j}\right)}{\pi^{j}\left(w^{j}\right)}
$$

or $\beta_{i}=\beta_{j}$ and $\pi^{i}\left(w^{j}\right)=\pi^{j}\left(w^{j}\right)$ for all $w^{j} \in W^{j}$. If the constraints of any agent $i \geq 2$ are both relevant in the limit and relevant w.r.t. those of $i-1$, agents 1 and 2 a.s. survive. If, in 
addition, for every $j \in\{2 \ldots n-1\}$ such that the survival indices of $j$ and $j+1$ are distinct, all $w^{j+1} \in W^{j+1}$ and all $w^{j} \subseteq w^{j+1}, \pi^{j}\left(w^{j} \mid w^{j+1}\right)=\pi\left(w^{j} \mid w^{j+1}\right)$, all agents a.s. survive.

The proposition considers agents with access to sets of assets ordered with respect to inclusion. Furthermore, less constrained agents have lower survival indices than more constrained agents. We impose the condition that the constraints of agents with lower survival indices are both relevant in the limit and relevant w.r.t. those with the next finer partition. ${ }^{11}$ In such an economy, the two agents with lowest survival indices, 1 and 2, a.s. survive. Requiring that agents' conditional beliefs $\pi^{j}\left(w^{j} \mid w^{j+1}\right)$ w.r.t. the next coarser partition are correct ${ }^{12}$ further implies that all agent a.s. survive regardless of the value of their survival indices. This result is interesting because it shows that agents with less financial constraints can survive even when their survival index is not maximal in the economy.

This requires, however, that for $i \geq 2$, agents' constraints are relevant even in the limit. In such a scenario, the constrained agents cannot consume the entire endowment of the economy: such a consumption stream would violate their financial constraints. Hence, it is the agents with lower survival indexes but weaker constraints who ensure that the markets clear. They consume the "leftovers" of the constrained agents and, thus, the fact that constraints are relevant ensures that they survive a.s.

Moreover, the least constrained agent 1 can survive in the presence of agents with stronger constraints and correct beliefs even if his beliefs about the contingencies on which others cannot trade are wrong. For example, in Example 1, Bob's constraints are relevant in the limit, whereas Ann's are not. When only Ann and Bob are present in the economy, given equal discount factors, Ann will survive if she and Bob assign equal probabilities to the events $w_{1}^{B}=\left\{s_{1} ; s_{3}\right\}$ and $w_{2}^{B}=\left\{s_{2} ; s_{4}\right\}$, regardless of whether her estimates about the probabilities of the individual states $s_{1}, s_{2}, s_{3}$, or $s_{4}$ are correct.

Our last two propositions in this section examine an economy in which the agents' constraints are not necessarily comparable. The economy discussed in Example 2 is an example of such a situation. In this economy, the sets of assets to which Bob and Clara have access are not ordered with respect to inclusion.

We now provide a formal definition of economies with nonnested financial constraints. Note that, for this definition, we use the sets consisting of generalized unit securities $\tilde{A}^{i}$.

DEFINITION 5Agents $i$ and $j$ have nonnested financial constraints if there are states ${ }^{13} s$, $s^{\prime}, s^{\prime \prime}, s^{\prime \prime \prime} \in S$ such that the following statements hold:

- There is an asset $a^{\prime} \in \tilde{A}^{i}$ such that $a^{\prime}(s) \neq a^{\prime}\left(s^{\prime}\right)$ and for any asset $a \in \tilde{A}^{i}, a\left(s^{\prime \prime}\right)=$ $a\left(s^{\prime \prime \prime}\right)$.

- There is an asset $a^{\prime \prime} \in \tilde{A}^{j}$ such that $a^{\prime \prime}\left(s^{\prime \prime}\right) \neq a^{\prime}\left(s^{\prime \prime \prime}\right)$ and for any asset $a \in \tilde{A}^{j}, a(s)=$ $a\left(s^{\prime}\right)$.

\footnotetext{
${ }^{11}$ The extension of Proposition 6 to the case where the constraints of some agent $i$ are irrelevant with respect to those of agent $i-1$ requires the examination of multiple cases. For example, it is easy to show that agents with irrelevant constraints weaker than those of agent 1 and survival indices lower than that of agent 1 a.s. vanish. Lemma 14 in Appendix A further shows that if an agent's constraints are irrelevant w.r.t. those of the agent with the next finest partition, but his survival index is strictly lower, this agent a.s. vanishes.

${ }^{12}$ Since $\pi^{j}\left(w^{j+1}\right)$ is, in general, incorrect, this does not imply that $\pi^{j}\left(w^{j}\right)$ is correct.

${ }^{13}$ The definition does not require the four states to be distinct and, thus, also applies to economies with only three states, where one can set $s^{\prime}=s^{\prime \prime}$. However, requiring $s^{\prime}=s^{\prime \prime}$ is, in general, too restrictive for our purposes, since it excludes, for example, an economy, in which $W^{i}=\left\{\{s\} ;\left\{s^{\prime}\right\} ;\left\{s^{\prime \prime} ; s^{\prime \prime \prime}\right\}\right\}$ and $W^{j}=\left\{\left\{s ; s^{\prime}\right\} ;\left\{s^{\prime \prime}\right\} ;\left\{s^{\prime \prime \prime}\right\}\right\}$. Indeed, choose any three states (e.g., $s, s^{\prime \prime}$, and $s^{\prime \prime \prime}$ ) and note that at least one of the agents (here, $j$ ) can trade among any of the three states, and, hence, the definition of nonnested constraints would not apply, contrary to intuition. In economies with only two states, agents' partitions are trivially nested.
} 
If the states $s$ and $s^{\prime}$ satisfy this definition, then we will say that $i$ can trade between $s$ and $s^{\prime}$, whereas $j$ cannot.

We will say that agents in the economy have nonnested financial constraints if, for each agent $i$, there are states $s$ and $s^{\prime} \in S$ between which $i$ can trade, but between which no other agent in the economy can trade.

Our first result shows that whenever an agent is the only one in the economy capable of trading between some relevant contingencies, he survives regardless of his beliefs and discount factor, and regardless of the financial constraints of the other agents.

PROPOSITION 7: Consider an economy with differential financial constraints and assume that for an agent $j$, there are states $s(j)$ and $s^{\prime}(j) \in S$ such that the financial constraints of $j$ and any other agent $i \in I \backslash\{j\}$ are nonnested and $j$ can trade between $s(j)$ and $s^{\prime}(j)$, whereas $i$ cannot. Assume that condition (4) holds for $s(j)$ and $s^{\prime}(j)$. Then agent $j$ survives a.s. In particular, if the condition above is satisfied for every agent $j \in I$, then all agents survive a.s.

This result is of special interest in view of Example 2 above. In particular, consider agents who are exposed to some nontradable idiosyncratic risk such as labor income. While the agent $j$ in question can be seen as owning the asset corresponding to his labor income stream, other agents cannot trade in this asset. That is, there are states $s(j)$ (for example, a state where $j$ is employed) and $s^{\prime}(j)$ ( $j$ is unemployed), which are distinct in $j$ 's partition, but which no other agent in the economy can trade on. Provided that the difference in payoffs in these states is bounded away from 0 in the limit, $j$ 's consumption will be strictly positive i.o. on almost every path and, hence, $j$ will survive a.s., regardless of his beliefs or discount factor.

A special case of Proposition 7 is that in which the intersection of the sets of assets to which agents have access is empty, that is, $\bigcap_{i \in I} A^{i}=\emptyset$. In this case, the finest partition that is coarser than all state partitions $\left(\Omega^{i}\right)_{i \in I}$ is the trivial partition. Hence, no trade across states occurs in equilibrium independently of agents' beliefs or on their discount factors. All agents survive a.s.

Our last result introduces an unconstrained agent with correct beliefs into the economy from Proposition 7. The presence of such an agent will cause all constrained agents with incorrect beliefs or lower discount factors to vanish a.s. However, we show that as long as the constraints are nonnested, and the constrained agents have correct beliefs and discount factors identical to that of the unconstrained agent, they survive a.s.

PROPOSITION 8: Take an economy with differential financial constraints and a set of agents $I^{\prime} \cup\{j\}\left(j \notin I^{\prime}\right)$. Suppose that the conditions of Proposition 7 are satisfied for the set $I^{\prime}$. Let $j$ be an unconstrained agent. Suppose that all agents have identical discount factors and correct beliefs. Then all agents survive a.s.

The results of this section have shown that markets do not select specifically for less constrained agents. However, when agents differ with respect to their beliefs and discount factors, less constrained agents have an advantage in that they can survive even when their beliefs are incorrect and their discount factor is smaller than that of less constrained agents. This implies that economies with differential financial constraints can exhibit limited risk sharing, biased state prices, and lower saving rates as compared to economies with identical financial constraints across agents. When the partitions are nonnested, agents with differential constraints survive. This can reduce the amount of trade in the economy. 
The next section discusses the impact of a relaxation of the agent's financial constraints on his survival.

\section{RELAXING CONSTRAINTS}

In our analysis so far, we have assumed that trade occurs only once, in period 0 , and that agents cannot retrade the resulting equilibrium allocation. In this section, we will consider what happens if constraints are relaxed. This might correspond, for instance, to a case of financial deregulation, where certain classes of investors are allowed to trade in assets that were previously unavailable to them. Alternatively, an agent may choose to invest through a less regulated intermediary, such as a hedge fund, in preference to, say, a mutual fund constrained to choose among investment grade securities. A particularly relevant case is that of a switch from defined benefit pensions to defined contribution funds. In this case, employees who previously held a nontradable claim to a pension payable on retirement receive access to funds that they must allocate over a potentially diverse portfolio. Since this is an exogenous change, beliefs about the possible returns on assets may have been formed on the basis of limited information and consideration.

A contrasting case is that of endogenous action ${ }^{14}$ by agents to remove or relax constraints on their investment choices as a result of increased awareness ${ }^{15}$ of investment possibilities. In such cases, agents might have beliefs based on research into the past performance and future prospects of the assets that become available. Such beliefs may be closer to those of agents already trading in the market, and ideally close to the most accurate beliefs of agents in the market.

For simplicity, we will consider a onetime change in constraints, such as allowing an agent to trade in an asset previously unavailable to him. In state space terms, this will mean that at node $\sigma_{t^{*}}^{*}$, agent $i$ gains access to a (weakly) finer partition of his state space, call this partition, $\Omega^{* i}$. Let $W^{* i}$ be the partition over states corresponding to the relaxed financial constraints $\Omega^{* i}$ of agent $i$ with a representative element $w^{* i}$.

Let $\Omega_{\sigma_{t^{*}}^{*}}$ denote the set of all (infinite) paths $\sigma^{*}$ with initial node $\sigma_{t^{*}}^{*}$ and let $\Omega_{\sigma_{t^{*}}^{* i}}^{* i}$ denote the set of all (infinite) paths $\omega^{* i}$ with initial node $\omega_{t^{*}}^{* i}$ such that $\sigma_{t^{*}}^{*} \in \omega_{t^{*}}^{* i}$. Intuitively, $\Omega_{\sigma_{t^{*}}^{* i}}^{* i}$ is the set of paths on which $i$ can trade given his relaxed financial constraints and given that the economy is in node $\sigma_{t^{*}}^{*}$.

Gaining access to a larger set of assets and, thus, to trades on a finer partition of the state space will require the agent to assign probabilities to the finer contingencies. Let $i$ 's probability distribution on $\left(\Omega^{* i} ; \mathcal{F}^{* i}\right)$ be denoted by $\pi^{* i}$. We will require that the revised beliefs are consistent with $i$ 's initial beliefs in the sense that $\pi^{i}\left(\omega_{t}^{i}\right)=$ $\sum_{\left\{\omega_{t}^{* i} \in \Omega^{* i} \mid \omega_{t}^{* i} \subseteq \omega_{t}^{i}\right\}} \pi^{* i}\left(\omega_{t}^{* i}\right)$. In particular, the revised beliefs will be consistent while satisfying the i.i.d. property if

$$
\begin{aligned}
\pi^{* i}\left(w^{* i} \mid \omega_{t}^{* i}\right) & =\pi^{* i}\left(w^{* i}\right) \text { and } \\
\pi^{i}\left(w^{i}\right) & =\sum_{\left\{w^{* i} \in W^{* i} \mid w^{* i} \subseteq w^{i}\right\}} \pi^{* i}\left(w^{* i}\right)
\end{aligned}
$$

\footnotetext{
${ }^{14}$ The distinction between exogenous and endogenous relaxation of a constraint was suggested to us by a referee, to whom we are grateful.

${ }^{15}$ There is a growing literature on changes in awareness. See Grant and Quiggin (2013), Karni and Vierø (2013, 2017), and Schipper (2016).
} 
for all possible continuations of $\omega_{t^{*}}^{* i}$ and $\omega_{t}^{* i}$. Note, however, that the beliefs on the finer partition $\pi^{* i}$ are not uniquely determined by the initial beliefs.

At $\sigma_{t^{*}}^{*}$, agents might want to reoptimize, taking into account their extended trade opportunities. To compute the new equilibrium at $\sigma_{t^{*}}^{*}$, we will take the initial equilibrium allocation to be the agents' initial endowments. We will compute the new allocation and the new equilibrium prices for the economy that starts at $\sigma_{t^{*}}^{*}$, taking into account that the consumption of agent $i$ now has to be measurable with respect to the finer partition $\Omega^{* i}$. To make sure that this reoptimization is meaningful, we have to impose a further assumption on the structure of the economy. To formulate the assumption, we say that an agent $i$ can trade on $\sigma_{t^{*}}^{*}$ if there is an $\omega_{t^{*}}^{* i} \in \Omega^{* i}$ such that $\omega_{t^{*}}^{*}=\left\{\sigma_{t^{*}}^{*}\right\}$.

ASSUMPTION Either of the following statements holds.

(i) All agents in the economy can trade on $\sigma_{t^{*}}^{*}$.

(ii) The agents' partitions are nested, at least one of the agents in the economy can trade on $\sigma_{t^{*}}^{*}$, and for each $k \in I$ who cannot trade on $\sigma_{t^{*}}^{*}$, any path $\omega^{* k} \in \Omega^{* k}$ such that $\sigma_{t^{*}}^{*} \in \omega_{t^{*}}^{* k}$, and any $t>t^{*}$, we have

$$
\min _{\sigma \in \omega^{* k}} e\left(\sigma_{t}\right)=\min _{\sigma \in \omega^{* k} \mid \sigma_{t^{*}=\sigma_{t^{*}}^{*}}} e\left(\sigma_{t}\right) .
$$

The intuition of the last point of the assumption is that we wish the set of paths passing through the node $\sigma_{t^{*}}^{*}$ to be similar to the set of paths that pass through the same cell of agent $k$ 's partition with respect to the lower bounds on the aggregate endowment.

Consider the economy from Example 1 consisting of Ann and Bob, and assume that endowments are i.i.d. over time. While there is no node besides $\sigma_{0}$ that both agents can trade on, at every node $\sigma_{t}$, Ann can trade on the node. Now consider a node $\sigma_{t^{*}}^{*}$ at which $B$ 's financial constraints are relaxed to $W^{* B}=\left\{\left\{s_{1} ; s_{3}\right\} ;\left\{s_{2}\right\} ;\left\{s_{4}\right\}\right\}$ and the corresponding $\Omega^{* B}=\prod_{\tau=0}^{\infty} W^{* B}$. Consider an element of $B$ 's new partition $\omega_{t}^{* B} \in \Omega^{* B}$, which is a continuation of the path $\omega_{t^{*}}^{* B}$ and, hence, $\sigma_{t^{*}}^{*} \in \omega_{t^{*}}^{* B}$. Recall that $s\left(\sigma_{t}\right)$ (respectively $w^{* B}\left(\sigma_{t}\right)$ ) stands for the state (respectively, the element of $B$ 's new partition $W^{* B}$ ) realized on path $\sigma$ in period $t$. By the construction of $\Omega^{* B}$, if $\sigma_{t}$ and $\sigma_{t}^{\prime} \in \omega_{t}^{* B}$ for some $t>t^{*}$, then $w^{* B}\left(\sigma_{t}\right)=w^{* B}\left(\sigma_{t}^{\prime}\right)$. Hence, either $s\left(\sigma_{t}\right)=s\left(\sigma_{t}^{\prime}\right)=s_{2}$, or $s\left(\sigma_{t}\right)=s\left(\sigma_{t}^{\prime}\right)=s_{4}$, or $s\left(\sigma_{t}\right) \in\left\{s_{1} ; s_{3}\right\}$ and $s\left(\sigma_{t}^{\prime}\right) \in\left\{s_{1} ; s_{3}\right\}$. Suppose that $\sigma^{\prime}$ satisfies $\sigma_{t^{*}}^{\prime}=\sigma_{t^{*}}^{*}$. Since the economy is i.i.d., we then have either

$$
\begin{aligned}
e\left(\sigma_{t}^{\prime}\right) & =e\left(\sigma_{t}\right)=e\left(s_{2}\right), \quad \text { or } \\
e\left(\sigma_{t}^{\prime}\right) & =e\left(\sigma_{t}\right)=e\left(s_{4}\right), \quad \text { or } \\
\min _{s \in\left\{s_{1} ; s_{3}\right\}} e\left(\sigma_{t-1}^{\prime} ; s\right) & =\min _{s \in\left\{\left\{_{1} ; s_{3}\right\}\right.} e\left(\sigma_{t-1} ; s\right) .
\end{aligned}
$$

It then follows that

$$
\min _{\sigma \in \omega^{* k}} e\left(\sigma_{t}\right)=\min _{\sigma^{\prime} \in \omega^{* k} \mid \sigma_{t^{*}}^{\prime}=\sigma_{t^{*}}^{*}} e\left(\sigma_{t}^{\prime}\right)
$$

as required by Assumption 4 .

The role of Assumption 4 is to establish that for the purposes of equilibrium analysis upon relaxing some of the financial constraints, we can restrict attention to an "economy" that starts at $\sigma_{t^{*}}^{*}$ with some endowment determined by trades at $t=0$. In particular, the equilibrium of the economy at $\sigma_{t^{*}}^{*}$ depends only on the set of successors of $\sigma_{t^{*}}^{*}$ as opposed 
to events that are impossible given $\sigma_{t^{*}}^{*}$. Indeed, Proposition 9 demonstrates that the market treats nodes that are not successors of $\sigma_{t^{*}}^{*}$ as 0 probability events by assigning them 0 prices in equilibrium.

As explained above, this condition is not overly restrictive.

REMARK 3: An economy with nested partitions and i.i.d. total initial endowment, that is, $e\left(\sigma_{t} ; s\right)=e\left(\sigma_{t^{\prime}}^{\prime} ; s\right)$ for all $\sigma_{t}, \sigma_{t^{\prime}}^{\prime} \in \Omega$, satisfies Assumption 4 for every $\sigma_{t^{*}}^{*}$.

DEFINITION 6: Let $\left(c^{i}\right)_{i \in I}$ be an equilibrium allocation of the economy with differential financial constraints. An equilibrium with relaxed constraints $\left(\Omega^{* i}\right)_{i \in I}$ at $\sigma_{t^{*}}^{*}$ is an integrable price system $\left(p^{*}\left(\sigma_{t}\right)\right)_{\sigma_{t} \in \Omega_{\sigma_{t^{*}}^{*}}}$ and consumption streams $\left(c^{* i}: \Omega_{\sigma_{t^{*}}^{*}} \rightarrow \mathbb{R}_{+}\right)_{i \in I}$ such that (i) all consumers $i$ are maximizing their expected utility given the price system subject to choosing consumption streams measurable relative to their relaxed constraints $\Omega^{* i}$ and (ii) markets clear:

$$
\begin{gathered}
c^{* i}=\arg \max _{c^{* i}}\left\{u_{i}\left(c^{i}\left(\sigma_{t^{*}}^{*}\right)\right)+\sum_{t=t^{*}}^{\infty} \beta_{i}^{t} \sum_{\omega_{t}^{* i} \in \Omega_{\sigma_{t^{*}}^{* i}}} \pi^{* i}\left(\omega_{t}^{* i}\right) u_{i}\left(c^{* i}\left(\omega_{t}^{* i}\right)\right)\right. \\
\text { s.t. } \sum_{t \geq t^{*}} \sum_{\omega_{t}^{* i} \in \Omega_{\sigma_{t^{*}}^{* i}}} \sum_{\sigma_{t}^{*} \in \omega_{t}^{* i}} p^{*}\left(\sigma_{t}\right) c^{* i}\left(\omega_{t}^{* i}\right) \\
\left.\leq \sum_{t \geq t^{*}} \sum_{\omega_{t}^{i} \in \Omega_{\sigma_{t^{*}}^{i}}^{i}} \sum_{\sigma_{t} \in \omega_{t}^{i}} p^{*}\left(\sigma_{t}\right) c^{i}\left(\omega_{t}^{i}\right)\right\}, \\
\sum_{i \in I} c^{* i}\left(\sigma_{t}\right)=\sum_{i \in I} c^{i}\left(\sigma_{t}\right) \quad \forall \sigma_{t} \in \Omega_{\sigma_{t^{*}}^{*}}^{*}
\end{gathered}
$$

PROPOSITION 9: Under Assumptions 1-4, an equilibrium with relaxed constraints exists. Furthermore, in such an equilibrium, $p\left(\sigma_{t}\right)=0$ for all $\sigma_{t} \notin \Omega_{\sigma_{t^{*}}^{*}}$. Hence, the equilibrium consumption can be characterized by the first order condition (f.o.c.)

$$
\frac{u_{i}^{\prime}\left(c^{i}\left(\omega_{t}^{* i}\right)\right)}{\beta_{i} \pi^{i}\left(\omega_{t+1}^{* i} \mid \omega_{t}^{* i}\right) u_{i}^{\prime}\left(c^{i}\left(\omega_{t+1}^{* i}\right)\right)}=\frac{p^{*}\left(\omega_{t}^{* i}\right)}{p^{*}\left(\omega_{t+1}^{* i}\right)}=\frac{\sum_{\sigma_{t}^{*} \in \omega_{t}^{* i} \cap \Omega_{\sigma_{t^{*}}^{*}}} p^{*}\left(\sigma_{t}^{*}\right)}{\sum_{\sigma_{t+1}^{*} \in \omega_{t+1}^{* i} \cap \Omega_{\sigma_{t^{*}}^{*}}} p^{*}\left(\sigma_{t+1}^{*}\right)} .
$$

The proposition shows that the f.o.c.s that characterize the equilibrium upon retrading coincide with the respective f.o.c.s in an economy with an initial node $\sigma_{0}$ replaced by $\sigma_{t^{*}}^{*}$. Hence, we can use the results from Sections 3 and 4 to characterize the equilibrium and study survival of an agent whose constraints have been relaxed. As we know from Section 5, given identical discount factors, the revised beliefs $\pi^{* i}$ will play a crucial role for $i$ 's survival. The next result follows directly from Proposition 5.

COROLlARY 1: Suppose that the economy satisfies Assumptions 1-3. Consider a population of agents with access to sets of assets ordered with respect to inclusion, $A^{1} \supset A^{2} \cdots \supset A^{n}$ 
and, thus, nested partitions $\Omega^{1}$ strictly finer than $\Omega^{2} \ldots$ strictly finer than $\Omega^{n}$. Suppose that at a node $\sigma_{t^{*}}^{*}$ that satisfies Assumption 4, agent $i>1$ 's set of assets is extended to $A^{* i}$ and, thus, his partition is refined to $\Omega^{* i}$, such that the new set of partitions satisfies the nested property.

Let all agents have identical (but not necessarily correct) beliefs $\tilde{\pi}$ and identical discount factors. Unless

$$
\begin{gathered}
\sum_{w^{1} \in W^{1}} \pi\left(w^{1}\right) \ln \frac{\pi^{* i}\left(w^{1}\right)}{\tilde{\pi}\left(w^{1}\right)} \geq 0 \quad \text { if } A^{* i} \supseteq A^{1} \quad \text { and } \\
\sum_{w^{* i} \in W^{* i}} \pi\left(w^{* i}\right) \ln \frac{\pi^{* i}\left(w^{* i}\right)}{\tilde{\pi}\left(w^{* i}\right)} \geq 0 \quad \text { if } A^{* i} \subset A^{1},
\end{gathered}
$$

i a.s. vanishes.

To understand the result, note that two cases are possible. First, $i$ 's new set of assets can be the largest (and, thus, his partition is the finest) in the population, $A^{* i} \supseteq A^{1}$. Since the constraints on agent 1 are not relevant in the economy, in the limit, agent $i$ 's consumption will be measurable w.r.t. agent 1's partition. Hence, $i$ cannot survive (relative to 1) unless he adopts beliefs at least as close to the truth as those of 1 on the finer partition. Second, $i$ 's new set $A^{* i}$ could be smaller than $A^{1}, A^{* i} \subset A^{1}$, implying that $\Omega^{* i}$ is coarser than $\Omega^{1}$. In this case, Proposition 5 implies that $i$ will a.s. vanish relative to agent 1 unless $i$ adopts beliefs at least as close to the truth as 1 .

The requirement that the agent adopt beliefs at least as close to the truth on the new partition as those of the agents with finer partitions might not be easy to satisfy. Unless the agent has a good understanding of the underlying uncertainty, the probability of guessing by chance a distribution that satisfies the condition is strictly less than 1 . Hence, even though an increase in an agent's opportunities for trade increases his welfare (see Proposition 4), this increase in utility comes at the cost of a positive probability of vanishing. If all agents have correct beliefs w.r.t. their initial partitions, $\tilde{\pi}=\pi$, "guessing the correct probability distribution"16 is a 0 -probability event. In contrast, an agent who can only trade in bonds, for whom $W^{i}=\{\{s \in S\}\}$, trivially has correct beliefs and survives.

We have shown that while markets with less constrained agents provide more opportunities for risk sharing ${ }^{17}$ they also pose greater risk for the survival of traders who might misjudge probabilities.

This issue has arisen in the literature on robust investment strategies for hedge funds. Eichhorn, Gupta, and Stubbs (1998) examine strategies that yield "satisfactory" returns for a range of beliefs, at the cost of being suboptimal for any precise belief. Eichhorn et al. conclude that "one plausible explanation for why investors constrain certain asset classes may arise because of differences in their relative confidence in the precision of the inputs."

\footnotetext{
${ }^{16}$ The issue of learning the true probabilities is beyond the scope of this paper. Nevertheless, we note that a straightforward corollary to Theorem 5 in Blume and Easley (2006) is that an agent who uses Bayesian updating to learn the correct probabilities will vanish in the presence of agents with finer partitions and correct beliefs. Another possibility suggested by an anonymous referee is for the investor to try to infer the other agents' distributions from prices. Sciubba (2005) studies survival in economies à la Grossman and Stiglitz (1980) and demonstrates that when information acquisition has small but strictly positive cost, uninformed traders can survive, implying that prices do not fully reveal all the available information even in the limit.

${ }^{17}$ Simsek (2013) points out that when traders have heterogeneous beliefs, the benefits of financial innovation in terms of risk sharing might be offset by the increased portfolio risk resulting from speculative trades.
} 
Interestingly, differential survival creates econometric difficulties for the ex post assessment of investment strategies for hedge funds. The most obvious such problem is that high-risk strategies are likely to yield higher than average returns for the subset of investors who follow such strategies and survive, even in the absence of any ex ante expectation of higher returns. Survival bias also poses a problem in assessing the persistence of hedge fund returns (Baqueroy, ter Horstz, and Verbeek (2005)). Persistence is relevant in the current context, since only persistent differences in performance can produce almost sure differential survival.

\section{CONCLUDING COMMENTS}

In the standard model of financial markets, all agents have access to the same set of financial assets. In this context, the analysis of Blume and Easley (2006) shows that if markets are complete, differences in prior beliefs are, ultimately, irrelevant since only agents with correct beliefs will survive. In contrast, in incomplete markets, agents with incorrect beliefs can survive, whereas agents with correct beliefs might vanish; see Coury and Sciubba (2012) and Beker and Chattopadhyay (2010).

In contrast, we examine markets in which agents face differential financial constraints and demonstrate the survival of agents with both differing beliefs and differing financial constraints. On the one hand, less constrained agents may survive, even when their beliefs are less accurate than those of others. Conversely, more constrained agents (those with a coarser partition of the state space) will survive if their beliefs regarding the coarser state space on which they can trade are accurate. Moreover, the cognitive and information requirements to form accurate beliefs about the payoffs of a constrained set of assets (on a coarse partition of the state space) are less demanding than the requirements for accurate probabilities regarding the full set of assets and, thus, the full set of economically relevant states. In particular, agents with nonstochastic endowments and minimal access to financial markets who invest only in bonds, will survive a.s., though they will forgo consumption opportunities available from insuring others.

In the main part of the paper, we concentrate on the case in which agents only trade at time 0 . This greatly simplifies the analysis and has the advantage of obtaining a clear-cut criterion for survival that relies on direct comparisons of the individual financial constraints, beliefs, and discount factors. This analysis mimics the approach of Sandroni (2005) by extending it to the case in which consumption, but not trade, is conditional on the sequential revelation of information. In Appendix A, we derive a criterion for survival with differential financial constraints for the case of sequential trade and show that it involves complex interactions between constraints, beliefs, and discount factors. Nevertheless, the two main insights gained from the case of period-0 trade remain true with sequential trade: (i) an agent who is more financially constrained relative to others in the economy need not vanish; in fact such constraints might allow an agent to survive even if he has wrong beliefs on the probabilities of states across which he is not allowed to trade; (ii) differential financial constraints allow for heterogeneity in beliefs, and discount factors to persist and affect prices in the long run, thus providing a more realistic scenario for the study of financial markets and, more generally, macroeconomic models with agent heterogeneity. 


\section{APPENDIX A: SEQUENTIAL TRADING}

In this appendix, we extend the analysis of the model to sequential trade. We use the model described in Section 3.1 to model the underlying uncertainty. The asset structure, however, has to be redefined as follows.

\section{A.1. Assets and Differential Financial Constraints}

Let $W^{0}$ be the partition of the state space $S$ as introduced in Section 3.2. For a given node $\sigma_{t} \in \Omega_{t}$, we consider a set of one-period-lived assets born at $\sigma_{t}$, available in 0 supply, which pay on the direct successors of $\sigma_{t}$ according to

$$
A_{\sigma_{t}}^{0}=\left\{a_{\sigma_{t} ; w}(s)=\left\{\begin{array}{ll}
1 & \text { if } s\left(\sigma_{t+1}\right) \in w, \\
0 & \text { else }
\end{array}\right\} .\right.
$$

That is, asset $a_{\sigma_{t} ; w}$ pays exactly 1 unit in those states $s$ following $\sigma_{t}$ that are in $w ; A_{\sigma_{t}}^{0}$ is the set of assets available to the economy as a whole. While it is convenient to keep the node $\sigma_{t}$ as the reference to the state at which an asset is traded, the partition $W^{0}$ does not depend on $\sigma_{t}$ and, hence, the asset structure remains constant over time. Markets generated by such a partition might be dynamically complete or incomplete.

Let $W^{i}$ be the partition of the state space corresponding to agent $i$. In our discussion of sequential trade, we will restrict attention to the case of nested partitions, that is, the case in which agents' partitions can be ordered from the finest to the coarsest. Assume, w.l.o.g. that for $i, j \in\{1 \ldots n\}, j>i$ implies that $j$ 's partition is coarser than that of $i$, and let agent 1's partition ${ }^{18} W^{1}=W^{0}$. Analogously to the case of period-0 trade, we wish to assume that at each node $\sigma_{t}$, agent $i$ has access to one-period-lived assets that span the partition $W^{i}$. However, certain complications arise.

In the sequel, we use the definition (and the proof of existence) of an equilibrium with implicit debt constraints in Magill and Quinzii (1994). Their proof of existence of an equilibrium does not require agents to have access to the same set of assets. However, it requires the assets in the economy to be linearly independent. This, in turn, means that we cannot simply endow each agent $i$ with the set of generalized unit securities, paying conditionally on the elements of $i$ 's own partition, as well as on the elements of the partitions of more constrained agents. Nevertheless, in Guerdjikova and Quiggin (2019), we show that we can construct the set of assets $\tilde{A}_{\sigma_{t}}^{0}$ for the economy and $\tilde{A}_{\sigma_{t}}^{i}$ available to each agent in such a way that (i) each agent $i$ has access to a linearly independent set of generalized unit securities that span the partition $W^{i}$, (ii) each agent $i$ has access to all assets available to agents with coarser partitions $j>i$ and each agent has access to the bond, and (iii) the set of all assets in the economy is linearly independent, contains the bond, and spans $W^{0}$.

For every $\sigma_{t}$, we set $\tilde{A}_{\sigma_{t}}^{0}=\bigcup_{i=1}^{n} \tilde{A}_{\sigma_{t}}^{i}$ to be the set of all assets in the economy at $\sigma_{t}$, $\tilde{A}^{i}=\bigcup_{\sigma_{t}} \tilde{A}_{\sigma_{t}}^{i}$ to be the set of all assets available to agent $i$, and $\tilde{A}^{0}=\bigcup_{i=1}^{n} \tilde{A}^{i}$ to be the set of all assets in the economy. An asset will be identified by the node at which it is born, $\sigma_{t}$, and the subset, $w$ of $S$, on which it pays, and we write $a_{\left(\sigma_{t} ; w\right)}$ for a representative element of $\tilde{A}_{\sigma_{t}}^{0}$.

\footnotetext{
${ }^{18}$ Since assets are available in 0 -supply, assets that are inaccessible to any of the agents are irrelevant for the economy.
} 


\section{A.2. Consumption and Endowments}

We maintain Assumption 1 in the main text and slightly strengthen Assumptions 2 and 3 to ensure that every agent's initial endowment is uniformly bounded away from 0 and agents' one-step-ahead probabilities are uniformly bounded away from 0 for all $s \in S$.

ASSUMPTION 2': There are $m^{\prime}$ and $\tilde{m}, m^{\prime}>\tilde{m}>0$, such that $e^{i}\left(\sigma_{t}\right)>\tilde{m}>0$ for all $i$ and $\sigma_{t} \in \Omega$, and $\sum_{i \in I} e^{i}\left(\sigma_{t}\right)<m^{\prime}$ for all $\sigma_{t} \in \Omega$.

Assumption 3': For all $s \in S, \pi(s)>0$. Agents' beliefs on $(\Omega ; \mathcal{F})$ are i.i.d. and for all $i \in I, \pi^{i}(s)>0$ for all $s \in S$.

With sequential trade, agents' beliefs on $S$ (as opposed to $W^{i}$ ) will matter for the analysis. Assumption $3^{\prime}$ implies the existence of $\underline{\pi}>0$ such that $\pi^{i}(s)>\underline{\pi}$ for all $i \in I$ and all $s \in S$.

Differently from the main part of the text, we do not require measurability of endowments and consumption streams w.r.t. the agent's partition. Indeed, a consequence of the equilibrium with sequential trading defined below is that even if initial endowments satisfy the measurability requirement, equilibrium consumption streams will, in general, not do so. We impose Assumptions 1, 2', and 3' for the remainder of Appendix A.

\section{A.3. Equilibrium}

We use the following definition of an equilibrium with implicit debt constraints as stated by Magill and Quinzii (1994).

DEFINITION An IDC (implicit debt constraint) equilibrium is defined by consumption streams $\left(c^{i}\left(\sigma_{t}\right): \Omega \rightarrow \mathbb{R}_{+}\right)_{i \in\{1 \ldots n\}}$, portfolio holdings $\left(\theta_{i}\left(\sigma_{t} ; a\right): \tilde{A}^{i} \rightarrow \mathbb{R}\right)_{i \in\{1 \ldots n\}}$, and asset prices $\left(q\left(\sigma_{t} ; \tilde{A}^{0}\right): \tilde{A}^{0} \rightarrow \mathbb{R}_{+}\right)$, such that the following statements hold:

(i) Consumers maximize utility $\sum_{t=0}^{\infty} \beta_{i}^{t} E_{\pi^{i}}\left[u_{i}\left(c^{i}\left(\sigma_{t}\right)\right)\right]$ subject to an implicit debt constraint:

$$
\begin{aligned}
B C= & \left\{c^{i}\left(\sigma_{t}\right) \in l_{\infty}^{+\Omega} \mid \text { there exists a } \theta_{i} \in l_{\infty}^{\tilde{A}^{i}} \text { s.t. for every } \sigma_{t} \in \Omega\right. \\
& c^{i}\left(\sigma_{t}\right)+\sum_{a \in \tilde{A}_{\sigma_{t}}^{i}} q\left(\sigma_{t} ; a\right) \theta_{i}\left(\sigma_{t} ; a\right) \leq e^{i}\left(\sigma_{t}\right)+\theta_{i}\left(\sigma_{t-1} ; a_{\left(\sigma_{t-1} ;\left(\sigma_{t}\right)\right)}\right) \\
& \text { and } \left.q \cdot \theta_{i} \in l_{\infty}^{\Omega}\right\} .
\end{aligned}
$$

(ii) Markets for the consumption good clear at each node: $\sum_{i \in I}\left[c^{i}\left(\sigma_{t}\right)-e^{i}\left(\sigma_{t}\right)\right]=0$ for all $\sigma_{t}$.

(iii) Asset markets clear at each node: $\sum_{i \in I} \theta_{i}\left(\sigma_{t} ; a\right)=0$ for all $\sigma_{t} \in \Omega$ and all $a \in \tilde{A}_{\sigma_{t}}^{0}$.

The linear independency of assets allows us to state that an equilibrium with implicit debt constraints of the economy exists; see Magill and Quinzii (1994). The equilibrium determines the price $q_{\left(\sigma_{t} ; w\right)}$ of any existing asset $a_{\left(\sigma_{t} ; w\right)} \in \tilde{A}_{\sigma_{t}}^{0}$ traded at node $\sigma_{t}$ and paying 1 unit on the event $\left(\sigma_{t} ; w\right)$. 
On the other hand, a simple arbitrage argument establishes that we can price any generalized Arrow security that pays on $\left(\sigma_{t} ; w^{i}\right)$ for some $w^{i} \in W^{i}, i \in\{0 ; 1 \ldots n\}$, even if it does not belong to $\tilde{A}^{0}$, using the prices of the existing assets.

This, in turn, allows us to state the equilibrium asset prices for those assets that pay on the market partition $W^{0}, q\left(\sigma_{t} ; w\right)$ and to restate the optimization problem of consumer $i$ in terms of the set of generalized unit securities (portfolios) that pay exactly on the partition $W^{i}$,

$$
\max _{c} \sum_{t=0}^{\infty} \beta_{i}^{t} E_{\pi^{i}}\left[u_{i}\left(c^{i}\left(\sigma_{t}\right)\right)\right],
$$

subject to the implicit debt constraint

$$
\begin{aligned}
B C= & \left\{c^{i}\left(\sigma_{t}\right) \in l_{\infty}^{+\Omega} \mid \text { there exists a } \theta_{i} \in l_{\infty}^{\Omega \times W^{i}} \text { s. t. for every } \sigma_{t} \in \Omega\right. \\
& c^{i}\left(\sigma_{t}\right)+\sum_{w \in W^{i}} q\left(\sigma_{t} ; w\right) \theta_{i}\left(\sigma_{t} ; w\right) \leq e^{i}\left(\sigma_{t}\right)+\theta_{i}\left(\sigma_{t-1} ; w\left(\sigma_{t}\right)\right) \\
& \text { and } \left.q \cdot \theta_{i} \in l_{\infty}^{\Omega}\right\} .
\end{aligned}
$$

The IDC equilibrium will satisfy the Euler equation for any agent $i$ and any asset paying on $\left(\sigma_{t} ; w^{i}\right), w^{i} \in W^{i}$ :

$$
q_{\left(\sigma_{t} ; w^{i}\right)}=\frac{\beta_{i} \sum_{s \in w^{i}} u_{i}^{\prime}\left(c^{i}\left(\sigma_{t} ; s\right)\right) \pi^{i}(s)}{u_{i}^{\prime}\left(\sigma_{t}\right)} .
$$

Furthermore, for any $j>i$ (that is, $W^{j}$ coarser than $W^{i}$ ) and $w^{j} \in W^{j}$, we have

$$
q_{\left(\sigma_{t} ; w^{j}\right)}=\frac{\beta_{i} \sum_{s \in w^{j}} u_{i}^{\prime}\left(c^{i}\left(\sigma_{t} ; s\right)\right) \pi^{i}(s)}{u_{i}^{\prime}\left(\sigma_{t}\right)} .
$$

We now collect some additional properties of the IDC equilibrium that will be useful for the analysis of survival in the next section.

We first show that equilibrium prices are uniformly bounded away from 0 and uniformly bounded from above.

LEMMA 1: In an IDC equilibrium, prices $q\left(\sigma_{t} ; w\right)$ are uniformly bounded away from 0 by $\underline{q}(w)>0$, and the price of the bond $\sum_{w \in W^{0}} q\left(\sigma_{t} ; w\right)$ is uniformly bounded above by $\bar{q}$ and below by $\underline{q}=\sum_{w \in W^{0}} \underline{q}(w)$.

Recall that an IDC equilibrium is characterized by a uniform bound on the value of debt $D$ (see Magill and Quinzii (1994)) such that

$$
\left|\sum_{w \in W^{i}} q\left(\sigma_{t} ; w\right) \theta_{i}\left(\sigma_{t} ; w\right)\right| \leq D \quad \text { for all } i \text { and } \pi \text {-a.s. all } \sigma_{t} \in \Omega \text {. }
$$


Combined with the uniform bounds on prices established in Lemma 1 and the fact that asset payoffs are linearly independent, this implies the following lemma.

LEMMA 2: If $D$ is the uniform bound on the value of debt in the IDC equilibrium, that is, $\left|\sum_{w \in W^{i}} q\left(\sigma_{t} ; w\right) \theta_{i}\left(\sigma_{t} ; w\right)\right| \leq D$ for all $i$ and $\pi$-a.s. all $\sigma_{t} \in \Omega$, then there is a uniform bound $N$ such that $\left|\theta_{i}\left(\sigma_{t} ; w\right)\right| \leq N$ for all $i$ and $\pi$-a.s. all $\sigma_{t} \in \Omega$.

The conditions listed so far are valid independently of the type of financial constraints. Consider two agents $i<j\left(W^{j}\right.$ is weakly coarser than $\left.W^{i}\right)$. For any given path $\sigma$,

$$
\frac{u_{i}^{\prime}\left(c^{i}\left(\sigma_{t}\right)\right)}{u_{j}^{\prime}\left(c^{j}\left(\sigma_{t}\right)\right)}=\frac{\beta_{i} \pi^{i}\left(w^{j}\left(\sigma_{t+1}\right)\right) \sum_{s \in w^{j}\left(\sigma_{t+1}\right)} u_{i}^{\prime}\left(c^{i}\left(\sigma_{t} ; s\right)\right) \pi^{i}\left(s \mid w^{j}\left(\sigma_{t+1}\right)\right)}{\beta_{j} \pi^{j}\left(w^{j}\left(\sigma_{t+1}\right)\right) \sum_{s \in w^{j}\left(\sigma_{t+1}\right)} u_{j}^{\prime}\left(c^{i}\left(\sigma_{t} ; s\right)\right) \pi^{j}\left(s \mid w^{j}\left(\sigma_{t+1}\right)\right)} .
$$

Define, analogously to Beker and Chattopadhyay (2010) (henceforth BC (2010)),

$$
\hat{r}_{i}^{j}\left(\sigma_{t}\right)=\frac{u_{i}^{\prime}\left(c^{i}\left(\sigma_{t}\right)\right)}{\sum_{s \in w^{j}\left(\sigma_{t}\right)} u_{i}^{\prime}\left(c^{i}\left(\sigma_{t-1} ; s\right)\right) \pi^{i}\left(s \mid w^{j}\left(\sigma_{t}\right)\right)} .
$$

Under the assumptions made on beliefs, utility functions, and endowments of the economy, the arguments in the proof of Proposition 1 in BC (2010) can be reproduced in our case, which implies the following analogue of their Proposition 1.

PROPOSITION 10: For any agent $i$ and agent $j>i$, that is, $W^{j}$ (weakly) coarser than $W^{i}$, $E_{\pi^{i}}\left[\hat{r}_{i}^{j}\left(\sigma_{t}\right) \mid \sigma_{t-1}\right]=1, \hat{r}_{i}^{j}\left(\sigma_{t}\right)>0$ and $\sup _{\sigma_{t} \in \Omega} \hat{r}_{i}^{j}\left(\sigma_{t}\right)<\infty$. Also, there is a random variable $R_{i}^{j}$, which is nonnegative and a.s. finite such that

$$
R_{i}^{j}(\sigma)=\lim _{T \rightarrow \infty} \prod_{t=1}^{T} \hat{r}_{i}^{j}\left(\sigma_{t}\right)
$$

In particular, note that the proposition applies equally when $W^{i}=W^{j}$ (as, for example, in the special case of identical financial constraints considered by BC (2010)).

Using the definition of $\hat{r}_{i}^{j}$, we obtain, analogously to Proposition 2 in BC (2010), the next proposition.

PROPOSITION 11: In equilibrium, the consumption of any two agents $i$ and $j$ such that $j$ is more constrained than $i$ satisfies

$$
\prod_{t=1}^{T+1} \hat{r}_{i}^{j}\left(\sigma_{t}\right)=\beta_{i}^{T+1} \frac{u_{i}^{\prime}\left(c^{i}\left(\sigma_{T+1}\right)\right)}{u_{i}^{\prime}\left(c^{i}\left(\sigma_{0}\right)\right)} \prod_{t=0}^{T} \frac{\pi^{i}\left(w^{j}\left(\sigma_{t+1}\right)\right)}{q\left(\sigma_{t} ; w^{j}\left(\sigma_{t+1}\right)\right)}
$$

and

$$
\frac{u_{i}^{\prime}\left(c^{i}\left(\sigma_{t+1}\right)\right)}{u_{j}^{\prime}\left(c^{j}\left(\sigma_{t+1}\right)\right)}=\frac{u_{i}^{\prime}\left(c^{i}\left(\sigma_{t}\right)\right)}{u_{j}^{\prime}\left(c^{j}\left(\sigma_{t}\right)\right)} \frac{\beta_{j}}{\beta_{i}} \frac{\pi^{j}\left(w^{j}\left(\sigma_{t+1}\right)\right)}{\pi^{i}\left(w^{j}\left(\sigma_{t+1}\right)\right)} \frac{\hat{r}_{i}^{j}\left(\sigma_{t}\right)}{\hat{r}_{j}^{j}\left(\sigma_{t}\right)} .
$$




\section{A.4. Survival in Economies With Sequential Trading}

Expression (5) is of particular interest, since it allows us to compare our framework to both the case of complete markets in Blume and Easley (2006) and the case of a single asset in BC (2010). With complete markets, as in Blume and Easley (2006), all agents have the finest partition and, hence, $\hat{r}_{j}^{j}\left(\sigma_{t}\right)=\hat{r}_{i}^{j}\left(\sigma_{t}\right)=1$ for all $\sigma_{t}$. It follows that

$$
\frac{u_{i}^{\prime}\left(c^{i}\left(\sigma_{t+1}\right)\right)}{u_{j}^{\prime}\left(c^{j}\left(\sigma_{t+1}\right)\right)}=\frac{u_{i}^{\prime}\left(c^{i}\left(\sigma_{t}\right)\right)}{u_{j}^{\prime}\left(c^{j}\left(\sigma_{t}\right)\right)} \frac{\beta_{j}}{\beta_{i}} \frac{\pi^{j}\left(w^{j}\left(\sigma_{t+1}\right)\right)}{\pi^{i}\left(w^{j}\left(\sigma_{t+1}\right)\right)},
$$

which implies that discount factors and beliefs only matter for survival.

In contrast, if all agents only have access to a single security as in BC (2010) (which in our case is restricted to be a bond), all agents have the trivial partition $\{S\}$, and since $\pi^{i}(S)=1$,

$$
\frac{u_{i}^{\prime}\left(c^{i}\left(\sigma_{t+1}\right)\right)}{u_{j}^{\prime}\left(c^{j}\left(\sigma_{t+1}\right)\right)}=\frac{u_{i}^{\prime}\left(c^{i}\left(\sigma_{t}\right)\right)}{u_{j}^{\prime}\left(c^{j}\left(\sigma_{t}\right)\right)} \frac{\beta_{j}}{\beta_{i}} \frac{\hat{r}_{i}^{j}\left(\sigma_{t}\right)}{\hat{r}_{j}^{j}\left(\sigma_{t}\right)} .
$$

Individual beliefs thus only enter this equation implicitly through $\hat{r}$.

When the partitions of the agents are nested, as in our case, beliefs on the coarser partition $W^{j}$ enter the equation explicitly and play a similar role as they do in the standard Blume and Easley (2006) complete market setting with correct beliefs on $W^{j}$ making the agent more likely to survive.

Consider the case in which aggregate uncertainty is measurable w.r.t. $W^{j}$ and all agents have identical beliefs. Then $\hat{r}_{i}^{j}\left(\sigma_{t}\right)=\hat{r}_{j}^{j}\left(\sigma_{t}\right)=1$ for all $\sigma_{t}$ and, hence, survival is determined only by the difference in discount factors. Clearly, in this case differential financial constraints are irrelevant for survival.

More generally, if

$$
\frac{\hat{r}_{i}^{j}\left(\sigma_{t+1}\right)}{\hat{r}_{j}^{j}\left(\sigma_{t+1}\right)}=\text { const }=1,
$$

we can think of markets as being "quasi-complete" w.r.t. $W^{j}$. Define $y^{j i}$ to be the ratio of marginal utilities of $j$ and $i$ :

$$
y^{j i}\left(\sigma_{t}\right)=\frac{u_{j}^{\prime}\left(\sigma_{t}\right)}{u_{i}^{\prime}\left(\sigma_{t}\right)} .
$$

An analogue of part (i) of Theorem 1 in BC (2010) shows that with equal discount factors, on those paths on which the variance of the ratio of agents' marginal utilities converges to 0 , the ratio $\frac{\hat{i}_{i}^{j}\left(\sigma_{t+1}\right)}{\hat{r}_{j}^{j}\left(\sigma_{t+1}\right)}$ indeed converges to 1 in the limit.

Proposition 12: Let $\beta_{i}=\beta_{j}$. Then

$$
\begin{aligned}
\lim _{t \rightarrow \infty} \frac{\pi^{j}\left(\sigma_{t} \mid \sigma_{t-1}\right)}{\pi^{i}\left(\sigma_{t} \mid \sigma_{t-1}\right)} \frac{y^{j i}\left(\sigma_{t}\right)}{y^{j i}\left(\sigma_{t-1}\right)} & =1 \quad \pi \text {-a.s. on } \sigma \text { satisfying } \\
\lim _{t \rightarrow \infty} \operatorname{Var}_{\pi}\left[\frac{\pi^{j}\left(\sigma_{t} \mid \sigma_{t-1}\right)}{\pi^{i}\left(\sigma_{t} \mid \sigma_{t-1}\right)} \frac{y^{j i}\left(\sigma_{t}\right)}{y^{j i}\left(\sigma_{t-1}\right)} \mid \sigma_{t-1}\right] & =0 .
\end{aligned}
$$


The results in the main part of the paper rely on agents' consumption streams being measurable w.r.t. their partitions. Such measurability in our case would imply $\hat{r}_{j}^{j}\left(\sigma_{t}\right)=1$ for all $\sigma_{t}$. We now show that with aggregate risk, if $j>i$, and if $i$ and $j$ have identical correct beliefs and discount factors, $j$ a.s. vanishes. In a second step, we provide conditions on beliefs and discount factors under which $j$ can survive.

To state the proposition, we first define an i.i.d. economy in which individual initial endowments depend only on the current state and are, thus, i.i.d.

DEFINITION 8: An i.i.d. economy has the property that $e^{i}\left(\sigma_{t} ; s\right)=e^{i}(s)$ for all $i \in I$, all $\sigma_{t} \in \Omega$, and all $s \in S$.

PROPOSITION 13Consider an i.i.d. economy with aggregate risk such that for some $\tilde{w}^{j} \in W^{j}$, there are $s, s^{\prime} \in \tilde{w}^{j}$ such that $e(s) \neq e\left(s^{\prime}\right)$. Suppose that $j$ 's equilibrium consumption ${ }^{19}$ satisfies

$$
\hat{r}_{j}^{j}\left(\sigma_{t}\right)=1 \text { for all } \sigma_{t} .
$$

Let i's partition $W^{i}$ be finer than that of $j, W^{j}$.

(a) If $\beta_{i}=\beta_{j}$ and $\pi^{i}=\pi^{j}=\pi$, then $j$ a.s. vanishes.

(b) If $I=\{i$; $j\}$ and if

$$
\ln \frac{\beta_{i}}{\beta_{j}}+\sum_{w^{j} \in W^{j}} \pi\left(w^{j}\right) \ln \frac{\pi^{i}\left(w^{j}\right)}{\pi^{j}\left(w^{j}\right)}<\sum_{s \in S} \pi(s) \ln \frac{\sum_{s \in w_{s}^{j}} u_{i}^{\prime}(e(s)) \pi^{i}\left(s \mid w_{s}^{j}\right)}{u_{i}^{\prime}(e(s))},
$$

then $\mathrm{j}$ a.s. survives.

Proposition 13 imposes measurability on j's equilibrium consumption. In general, this property will not hold in equilibrium. Our next result shows indeed that $j$ can vanish a.s. conditional on any given node only in a set of i.i.d. economies with measure 0 .

Proposition 14: Consider an i.i.d. economy with two agents. Assume that

$$
\beta_{i}<\frac{u_{i}^{\prime}\left(m^{\prime}\right)}{u_{i}^{\prime}(\tilde{m})}, \quad i \in\{1 ; 2\} .
$$

If the partition generated by agent $j$ 's financial constraints, $W^{j}$, has at least one element $\bar{w}^{j}$ that contains at least two distinct states $s_{\bar{w}^{j}} \neq s_{\bar{w}^{j}}^{\prime}$, then, except for a set of endowment processes with measure 0 , there is no node $\bar{\sigma}_{\bar{t}} \in \Omega$ such that an agent $i \in\{1 ; 2\}$ a.s. vanishes conditional on $\bar{\sigma}_{\bar{t}}$.

In economies with only two agents, there is no distinction between differential financial constraints and market incompleteness: the set of possible trades is restricted to those measurable w.r.t. to both agents' partitions. Hence, the condition imposed on the partition of agent $j$ implies that markets indeed are incomplete. The condition on the agents' discount factors further ensures that the equilibrium price of the bond in this economy is

\footnotetext{
${ }^{19}$ Note that we do not claim that such an equilibrium exists. For the case of two agents, and non-i.i.d. economies, BC (2010) provide a method of constructing economies in which equilibria satisfy this property.
} 
bounded strictly below $1, \bar{q}<1$. Under this condition, we show that none of the agents can vanish a.s. conditional on a node $\bar{\sigma}_{\bar{t}} \in \Omega$. Note that this result is independent of agents' beliefs and does not depend on the relative comparison of their discount factors or (trivially) on the type of financial constraints imposed as long as markets are incomplete.

To provide some intuition for the result, we discuss an example.

EXAMPLE 3: Consider an economy with $S=\left\{s_{1}, s_{2}, s_{3}\right\}, e\left(s_{1}\right)=4, e\left(s_{2}\right)=2$, and $e\left(s_{3}\right)=1$. The economy is i.i.d. with $\pi(s)>0$ for all $s$, and $W^{j}=\left\{\left\{s_{1} ; s_{2}\right\} ;\left\{s_{3}\right\}\right\}$ and $W^{i}=\left\{\left\{s_{1}\right\},\left\{s_{2}\right\},\left\{s_{3}\right\}\right\}$. Clearly, since there are only two agents in the economy, the constraint on $j$ 's trades together with market clearing implies that $i$ can also effectively only trade on $W^{j}$. Assume that ${ }^{20} u_{i}(\cdot)=\ln (\cdot)$ and $\pi^{i}(s)=\frac{1}{3}$ for all $s$, and let $e^{j}\left(s_{1}\right)=e^{j}\left(s_{2}\right)=1$ and $e^{j}\left(s_{3}\right)=\frac{1}{2}$.

We will argue that agent $j$ cannot vanish a.s. conditional on a node $\tilde{\sigma}_{\tilde{t}}$; the argument for $i$ is analogous. Suppose, thus, that conditional on some node $\tilde{\sigma}_{\tilde{t}}, j$ a.s. vanishes. In Lemma 18 we show that on paths emanating from $\tilde{\sigma}_{\tilde{t}}$, equilibrium asset prices converge to those in an economy with a representative agent $i: q^{i}\left(s ; w=\left\{s_{1} ; s_{2}\right\}\right)=\frac{1}{4} \beta_{i} e(s)$ and $q^{i}\left(s ; w=\left\{s_{3}\right\}\right)=\frac{1}{3} \beta_{i} e(s)$.

By assumption, $\beta_{i}<\frac{u_{i}^{\prime}\left(m^{\prime}\right)}{u_{i}^{\prime}(\tilde{m})}=\frac{1}{4}$, thus implying that the maximal limit price of the bond is $\frac{7}{3} \beta_{i}<1$.

Furthermore, since $j$ vanishes a.s. conditional on $\tilde{\sigma}_{\tilde{t}}, c^{j} \rightarrow 0$. So as to convey the intuition of the argument for this example, we will use directly the limit prices $q^{i}(s ; w)$ and the limit consumption of $j, c^{j}=0$. The precise argument, which takes into account the fact that conditional on $\tilde{\sigma}_{\tilde{t}}$, asset prices and $j$ 's consumption are eventually close, but do not coincide with their limit values, is spelled out in the proof of Proposition 14.

Since $j$ 's initial endowment is bounded below by $\frac{1}{2}$, to obtain 0 consumption, he has to either hold short positions in the relevant asset at the beginning of the period or, alternatively, use his entire initial endowment and dividends to buy assets instead. One possibility to accomplish this would be by holding a stationary portfolio $\theta^{*}(w)$ such that for all $s$ and the corresponding element of the partition $w_{s}$ such that $s \in w_{s}$,

$$
\theta^{*}\left(w_{s}\right)+e^{j}(s)=\sum_{\tilde{w} \in\left\{\left\{s_{1} ; s_{2}\right\} ;\left\{s_{3}\right\}\right\}} q^{i}(s ; \tilde{w}) \theta^{*}(\tilde{w})=\frac{1}{3} e(s) \beta_{i}\left[\frac{3}{4} \theta^{*}\left(\left\{s_{1} ; s_{2}\right\}\right)+\theta^{*}\left(\left\{s_{3}\right\}\right)\right]
$$

holds. Note, however, that this system is overdetermined whenever $j$ 's partition is not the finest one. Since $e\left(s_{1}\right) \neq e\left(s_{2}\right)$, it thus does not have a solution for $e^{j}\left(s_{1}\right)=e^{j}\left(s_{2}\right)=1$ (and, more generally, except for a measure 0-endowment processes).

Instead, we solve a subsystem taking one equation for each element of the partition (e.g., $s_{1}$ for $\left\{s_{1} ; s_{2}\right\}$ and, trivially, $s_{3}$ for $\left\{s_{3}\right\}$; such a subsystem has a unique solution except for a measure 0 -endowment processes):

$$
\begin{aligned}
\theta^{*}\left(\left\{s_{1} ; s_{2}\right\}\right)+1 & =\frac{4}{3} \beta_{i}\left[\frac{3}{4} \theta^{*}\left(\left\{s_{1} ; s_{2}\right\}\right)+\theta^{*}\left(\left\{s_{3}\right\}\right)\right], \\
\theta^{*}\left(\left\{s_{3}\right\}\right)+\frac{1}{2} & =\frac{1}{3} \beta_{i}\left[\frac{3}{4} \theta^{*}\left(\left\{s_{1} ; s_{2}\right\}\right)+\theta^{*}\left(\left\{s_{3}\right\}\right)\right] .
\end{aligned}
$$

\footnotetext{
${ }^{20}$ We provide only the specifications strictly necessary for the understanding of the argument. Agent $j$ 's beliefs and utility function do not impact the argument below and, hence, can be specified arbitrarily within the assumptions of the model.
} 
Intuitively, the solution of (6) is a "stationary state" in which $j$ has 0 consumption, while holding a constant portfolio along a path on which only states $s_{1}$ and $s_{3}$ occur. Clearly, such paths have a measure 0 . However, we will now show that if $j$ starts by holding a portfolio that deviates from $\theta^{*}$, his portfolio holdings will exceed any predefined boundary in finite time with strictly positive probability, thus violating the result of Lemma 2.

Indeed, consider the solution to $(6) \theta^{*}\left(\left\{s_{1} ; s_{2}\right\}\right)$ and $\theta^{*}\left(\left\{s_{3}\right\}\right)$. Suppose that at $\tilde{\sigma}_{\tilde{t}}$, $\theta_{j}\left(\tilde{\sigma}_{\tilde{t}} ;\left\{s_{1} ; s_{2}\right\}\right)=\theta^{*}\left(\left\{s_{1} ; s_{2}\right\}\right)+d$, where ${ }^{21} d>0$. Suppose, thus, that at $\tilde{t}+1, s_{1}$ is realized. Then, from $j$ 's budget constraint together with the condition that $c^{j}\left(\tilde{\sigma}_{\tilde{t}} ; s_{1}\right)=0, j$ 's portfolio $\theta_{j}$ has to satisfy

$$
\theta_{j}\left(\tilde{\sigma}_{\tilde{t}} ;\left\{s_{1} ; s_{2}\right\}\right)+1=\theta^{*}\left(\left\{s_{1} ; s_{2}\right\}\right)+d+1=\frac{4}{3} \beta_{i}\left[\frac{3}{4} \theta_{j}\left(\tilde{\sigma}_{\tilde{t}} ; s_{1} ;\left\{s_{1} ; s_{2}\right\}\right)+\theta_{j}\left(\tilde{\sigma}_{\tilde{t}} ; s_{1} ;\left\{s_{3}\right\}\right)\right] .
$$

Using the first equation in the system (6), we have

$$
\frac{3}{7} \theta_{j}\left(\tilde{\sigma}_{\tilde{t}} ; s_{1} ;\left\{s_{1} ; s_{2}\right\}\right)+\frac{4}{7} \theta_{j}\left(\tilde{\sigma}_{\tilde{t}} ; s_{1} ;\left\{s_{3}\right\}\right)=\frac{d}{\frac{7}{3} \beta}+\left[\frac{3}{7} \theta^{*}\left(\left\{s_{1} ; s_{2}\right\}\right)+\frac{4}{7} \theta^{*}\left(\left\{s_{3}\right\}\right)\right]
$$

and, hence, either

$$
\begin{aligned}
& \theta_{j}\left(\tilde{\sigma}_{\tilde{t}} ; s_{1} ;\left\{s_{1} ; s_{2}\right\}\right)>\frac{d}{\frac{7}{3} \beta_{i}}+\theta^{*}\left(\left\{s_{1} ; s_{2}\right\}\right)>d+\theta^{*}\left(\left\{s_{1} ; s_{2}\right\}\right) \quad \text { or } \\
& \theta_{j}\left(\tilde{\sigma}_{\tilde{t}} ; s_{1} ;\left\{s_{3}\right\}\right)>\frac{d}{\frac{7}{3} \beta_{i}}+\theta^{*}\left(\left\{s_{3}\right\}\right)>d+\theta^{*}\left(\left\{s_{3}\right\}\right),
\end{aligned}
$$

since (as shown above) $\frac{7}{3} \beta_{i}<1$.

Intuitively, since the assets bought by $j$ exceed the quantities in the "stationary state," since he receives both their dividends and his initial endowment, and since, by assumption, asset prices are lower than 1, the quantity he needs to buy in the subsequent period of at least one of the assets must also exceed the stationary quantity $\theta^{*}$, but this time by $\frac{d}{\frac{7}{3} \beta_{i}}>d$.

Proceeding inductively, one sees that one can choose a path of $\kappa$ subsequent realizations of the states $s_{1}$ and $s_{3}$, such that on this path, $j$ 's asset holdings in one of the two assets will exceed $\frac{d}{\left(\frac{7}{3} \beta_{i}\right)^{\kappa}}$. Thus, for sufficiently large $\kappa \mathrm{s}, j$ 's asset holdings will exceed any initially predetermined bound on assets. Since any sequence of states $s_{1}$ and $s_{3}$ of length $\kappa$ occurs with a strictly positive probability, this generates a contradiction to the hypothesis that $j$ a.s. vanishes in equilibrium. Hence, $\theta_{j}\left(\tilde{\sigma}_{\tilde{t}} ;\left\{s_{1} ; s_{2}\right\}\right)=\theta^{*}\left(\left\{s_{1} ; s_{2}\right\}\right)$ should hold.

However, by the same argument (exchanging $s_{1}$ with $s_{2}$ and considering the continuation $\left.\left(\tilde{\sigma}_{\tilde{t}} ; s_{2}\right)\right), \theta_{j}\left(\tilde{\sigma}_{\tilde{t}} ;\left\{s_{1} ; s_{2}\right\}\right)$ should be given by the solution $\theta^{* *}\left(\left\{s_{1} ; s_{2}\right\}\right)$ to

$$
\begin{aligned}
\theta^{* *}\left(\left\{s_{1} ; s_{2}\right\}\right)+1 & =\frac{2}{3} \beta_{i}\left[\frac{3}{4} \theta^{* *}\left(\left\{s_{1} ; s_{2}\right\}\right)+\theta^{* *}\left(\left\{s_{3}\right\}\right)\right], \\
\theta^{* *}\left(\left\{s_{3}\right\}\right)+\frac{1}{2} & =\frac{1}{3} \beta_{i}\left[\frac{3}{4} \theta^{* *}\left(\left\{s_{1} ; s_{2}\right\}\right)+\theta^{* *}\left(\left\{s_{3}\right\}\right)\right] .
\end{aligned}
$$

\footnotetext{
${ }^{21}$ The argument for $d<0$ is symmetric.
} 
But $\theta^{*}\left(\left\{s_{1} ; s_{2}\right\}\right) \neq \theta^{* *}\left(\left\{s_{1} ; s_{2}\right\}\right)$ (except for a set of endowments of measure 0$)$. Hence, starting with holdings $\theta^{*}\left(\left\{s_{1} ; s_{2}\right\}\right)$ implies a nonzero difference $d$ to $\theta^{* *}\left(\left\{s_{1} ; s_{2}\right\}\right)$, and one can find a finite sequence of states $s_{2}$ and $s_{3}$ generating the same contradiction as above.

As the example illustrates, the assumption of an i.i.d. economy plays two roles: first, it ensures that on paths on which $j$ vanishes, the limit asset price process depends only on the current state of the economy (this is also true in the Markov setting used by BC (2010)); second, it guarantees that as long as there is variation in the endowment process within one of the elements of the trading partition, this variation is persistent and a.s. occurs infinitely often on any given path, ${ }^{22}$ so that there is no unique stationary portfolio that makes $j$ 's consumption consistently converge to 0 .

While the result of Proposition 14 does not imply that the probability with which an agent vanishes is 0 , this set is (a) of measure less than 1 and (b) nowhere dense. ${ }^{23}$

The idea that a given type of agents (for example, those with wrong beliefs, more severe constraints, etc.) will survive or vanish except for a negligibly small set of histories can be formalized in two different ways: (a) in measure theoretic terms, these agents survive or vanish a.s.; (b) in topological terms, the set on which these agents vanish or survive is meager (a countable union of nowhere dense sets). That is, they are of Baire category 1.

In some relevant cases, these concepts coincide. Most obviously, if we represent the history as a binary expansion, those histories that eventually end in a repetitive pattern correspond to the rationals, which are both meager and of zero measure. These histories can be exploited by trading strategies, even for agents with wrong beliefs about the probability distribution of the generating process.

It turns out, however, that intuitions based on the rationals are misleading. In general, the concepts of measure and Baire category are orthogonal; see Marinacci (1994). We have therefore addressed both criteria.

Finally note that one can interpret the two agents in the proposition as types. In particular, we can replace each agent $i \in\{1 ; 2\}$ by a set of $n$ that is identical w.r.t. preferences, beliefs, discount factors, constraints, and initial endowments agents, and consider an equilibrium in which all agents of the same type behave identically. For such an equilibrium, no agent will vanish a.s. conditional on any node.

We conclude the analysis of sequential trading with the following result.

PROPOSITION 15: Consider an i.i.d. economy populated by a finite set of agents I and such that the uniform upper and lower bounds on equilibrium prices determined in Lemma 1 satisfy $\underline{q}<\bar{q}<1$. Suppose that for agent $i \in I$,

$$
\max _{w \in W^{i}} \min _{s \in w} e^{i}(s)<(1-\underline{q})(1-\bar{q}) \min _{w \in W^{i}} \max _{s \in w} e^{i}(s) .
$$

Furthermore, suppose that there is at least one agent in the economy with financial constraints leading to a partition at least as fine as $W^{i}$. Then there is no node $\bar{\sigma}_{\bar{t}}$ such that agent $i$ vanishes a.s. conditional on $\bar{\sigma}_{\bar{t}}$.

\footnotetext{
${ }^{22} \mathrm{An}$ examination of the proof suggests that the results extend to an economy in which endowments depend only on the current state, but beliefs are Markovian, provided that all one-step-ahead transitional probabilities are strictly positive. For general non-i.i.d. economies, the result need not, however, hold, as demonstrated by BC (2010).

${ }^{23}$ Rudin (1983) provides a method for the construction of such sets on the interval [0; 1], which is topologically equivalent to the set $\Omega$.
} 
Similarly to the previous proposition, the result uses a condition on the upper and lower bounds of prices in the economy as well as the initial endowment. As shown in Magill and Quinzii (1994) and Krebs (2004), these bounds depend on the parameters of the economy, such as the total initial endowment, the agent's preferences and discount factors, and the minimal probabilities $\underline{\pi}$. This suggests that for a given initial endowment for $i$, the rest of the parameters of the economy can be adjusted in such a way that the condition on $\bar{q}$ and $q$ is satisfied.

In particular, the condition relating the initial endowment of agent $i$ and the bounds on prices, $q$ and $\bar{q}$, precludes the case in which $i$ has access to a complete set of securities and, thus, his partition is $W^{i}=\left\{\{s\}_{s \in S}\right\}$. Indeed, (7) would then require

$$
\max _{s \in S} e^{i}(s)<(1-\underline{q})(1-\bar{q}) \min _{s \in S} e^{i}(s),
$$

which is excluded by $(1-q)(1-\bar{q})<1$. In contrast, if $i$ only has access to a bond, $W^{i}=$ $\{\{S\}\}$, the requirement in $(\overline{7})$ becomes

$$
\min _{s \in S} e^{i}(s)<(1-\underline{q})(1-\bar{q}) \max _{s \in S} e^{i}(s) .
$$

In particular, the proof of the proposition uses the fact that (7) implies that for $w^{1} \in W^{i}$ defined as

$$
w^{1} \in \underset{w \in W^{i}}{\arg \max _{s \in w}} \min ^{i}(s),
$$

$\max _{s \in w^{1}} e^{i}(s)$ strictly exceeds $\frac{\min _{s \in w^{1}} e^{i}(s)}{1-\bar{q}}$ and, hence, $w^{1}$ has at least two states leading to two distinct endowments for agent $i$.

Similarly to Theorem 2 in BC (2010), the two propositions that establish survival of agents with financial constraints do not use the fact that the (potentially vanishing) agent's consumption is optimal. Rather, they exploit the idea that financially constrained agents will be limited in their ability to sell their initial endowment. In particular, our results show that when constraints are nontrivial, an agent whose consumption goes to 0 a.s. conditional on a node $\bar{\sigma}_{\bar{t}}$ will have to hold arbitrarily large long or short positions in at least some of the assets in finite time. This, combined with the limits on equilibrium prices contradicts, as shown in Lemma 2, the uniform bounds on debt.

Differently from the results stated in the main part of the paper, in the case of sequential trade, we do not obtain clear-cut criteria for survival that can be reduced to direct comparisons of the individual financial constraints, beliefs, and discount factors. Instead, similarly to the results in BC (2010) for incomplete markets, we obtain a criterion for survival that combines in nontrivial fashion all of the above and suggests that the equilibrium dynamics in markets with differential financial constraints and sequential trade will be rather complex. Nevertheless, the two main insights gained from the case of period-0 trade remain true: (i) financial constraints per se do not harm the agent's chances to survive; (ii) differential financial constraints allow for heterogeneity in beliefs and discount factors in the long run.

\section{APPENDIX B: PROOFS}

\section{Proof of Proposition 1}

An equilibrium of the economy exists under the following conditions (Bewley (1972)): 
(i) The consumption sets are convex, Mackey-closed, and contained in the set of essentially bounded measurable functions.

(ii) The preferences of the agents are complete and transitive.

(iii) The better sets are convex and Mackey-closed.

(iv) The worse sets are closed in the norm topology.

(v) There exists a set of paths with strictly positive measure such that the preferences of all agents satisfy strict monotonicity on this set. That is, adding a constant to the payoff in each state and each period makes the agent strictly better off.

(vi) For all agents, the initial endowments are in the interior of the consumptions sets.

We can assume that the consumption set of an agent $i \in\{1 \ldots n\}$ is given by the sets of all essentially bounded measurable functions on $\Omega^{i}$ and, hence, satisfies condition (i). We can then define the function $V_{0}^{i}\left(c^{i}\right)$ on the set of all essentially bounded measurable functions on $\Omega$, while the measurability restriction with respect to $\Omega^{i}$ is imposed by the consumption set of $i$. Condition (ii) is then trivially satisfied. The convexity requirement in condition (iii) follows from the concavity of the utility function $u^{i}$. Further, $V_{0}^{i}$ is uniformly continuous and, hence, continuous with respect to the Mackey topology. This means that both the better and the worse sets are closed with respect to the Mackey topology and, hence, also to the norm topology. The second requirement in conditions (iii) and (iv) are therefore satisfied.

For condition (v) and an agent $i$, take the set of paths to be $\Omega$. Note that $V_{0}^{i}$ is monotonic. Take any consumption stream $c$. Adding a positive amount to $c$ strictly improves the act. Hence, the preferences of all agents are strictly monotonic on $\Omega$.

As for condition (vi), a careful examination of Bewley's (1972) proof shows that in the absence of production, this condition can be relaxed ${ }^{24}$ as in Assumption 2. We conclude that an equilibrium of the economy exists.

Note that the measurability condition on $i$ 's consumption ensures that $u_{i}^{\prime}\left(c^{i}\left(\sigma_{t}\right)\right)=$ $u_{i}^{\prime}\left(c^{i}\left(\sigma_{t}^{\prime}\right)\right)$ for all $\sigma_{t}, \sigma_{t}^{\prime} \in \Omega_{t}^{i}$. If $p(\cdot)$ is an equilibrium price system, then condition (2),

$$
\frac{u_{i}^{\prime}\left(c^{i}\left(\omega_{t}^{i}\right)\right)}{\beta_{i} \pi^{i}\left(\omega_{t+1}^{i} \mid \omega_{t}^{i}\right) u_{i}^{\prime}\left(c^{i}\left(\omega_{t+1}^{i}\right)\right)}=\frac{p\left(\omega_{t}^{i}\right)}{p\left(\omega_{t+1}^{i}\right)}=\frac{\sum_{\sigma_{t} \in \omega_{t}^{i}} p\left(\sigma_{t}\right)}{\sum_{\sigma_{t+1} \in \omega_{t+1}^{i}} p\left(\sigma_{t+1}\right)},
$$

is the first-order condition of agent $i$ 's maximization problem at state $\sigma_{t}$. Hence, it will be satisfied in any equilibrium in which agent $i$ chooses an interior allocation on all finite paths with positive probabilities. We now show that Assumptions 1-3 imply that the optimal consumption streams of all agents will be strictly positive on all finite paths that have positive probability. To show this, we demonstrate that the marginal rate of substitution between consumption at $\sigma_{0}$ and $\omega_{t}$ will always be strictly positive and finite, provided that the true probability of $\omega_{t}$ is positive.

Since the initial endowment is uniformly bounded above, so are all the consumption streams in equilibrium. Hence, by Assumption $1, u_{i}^{\prime}$ is always strictly positive. Furthermore, setting $c\left(\sigma_{0}\right)=0$ is not optimal, since, by Assumption 2, endowment is uniformly

\footnotetext{
${ }^{24}$ Indeed, Assumption 2 is sufficient for the existence of an equilibrium in a finite economy with no production as in Debreu (1962). The extension of the price functional of each such finite economy to the whole state space as in Bewley (1972, p. 521) only requires Assumption 2. With no production, the rest of the Bewley (1972) proof can be replicated under Assumption 2. In particular, the last argument on page 523 follows directly from equation (1) on page 522 and the strict positivity of individual endowments.
} 
bounded away from 0 , and by Assumption $1, u^{\prime}(0)=\infty$. Take an arbitrary $\omega_{t}^{i}$ such that $\pi\left(\omega_{t}^{i}\right)>0$ and, hence, by Assumption $3, \pi^{i}\left(\omega_{t}^{i}\right)>0$. If $c\left(\omega_{t}\right)=0$ and if $p\left(\sigma_{0}\right), p\left(\omega_{t}\right)>0$, an iteration on (2) gives

$$
\operatorname{MRS}^{i}\left(c^{i}\left(\sigma_{0}\right) ; c^{i}\left(\omega_{t}\right)\right)=\frac{u_{i}^{\prime}\left(c^{i}\left(\sigma_{0}\right)\right)}{\beta_{i}^{t} \pi^{i}\left(\omega_{t}^{i}\right) u_{i}^{\prime}\left(c^{i}\left(\omega_{t}^{i}\right)\right)}=0<\frac{p\left(\sigma_{0}\right)}{p\left(\omega_{t}\right)},
$$

which cannot hold in the optimum. Hence, $c^{i}\left(\omega_{t}\right)=0$ can only obtain if $\pi^{i}\left(\omega_{t}\right)=0$ or, by Assumption 2, if $\pi\left(\omega_{t}\right)=0$. We thus obtain that $i$ will have strictly positive consumption on all finite paths that have positive probability with respect to the truth. This, in turn, implies that the first-order condition will hold on all such paths.

\section{Derivations for Example 1}

\section{CLAIM 1: Neither of the two agents is insured against idiosyncratic risk in equilibrium.}

Proof: Let $u_{A}(\cdot)$ and $u_{B}(\cdot)$ be $A$ 's and $B$ 's concave von Neumann-Morgenstern utility. Standard expected utility maximization then gives the f.o.c.s for Ann,

$$
\frac{u_{A}^{\prime}\left(c^{A}(s)\right) \pi(s)}{u_{A}^{\prime}\left(c^{A}\left(s^{\prime}\right)\right) \pi\left(s^{\prime}\right)}=\frac{p_{s}}{p_{s^{\prime}}} \quad \text { for } s, s^{\prime} \in\{1 \ldots 4\},
$$

and for Bob $\left(\right.$ since $\left.c^{B}\left(s_{1}\right)=c^{B}\left(s_{3}\right), c^{B}\left(s_{2}\right)=c^{B}\left(s_{4}\right)\right)$,

$$
\frac{u_{B}^{\prime}\left(c^{B}\left(s_{1}\right)\right)\left(\pi\left(s_{1}\right)+\pi\left(s_{3}\right)\right)}{u_{B}^{\prime}\left(c^{B}\left(s_{2}\right)\right)\left(\pi\left(s_{2}\right)+\pi\left(s_{4}\right)\right)}=\frac{p_{1}+p_{3}}{p_{2}+p_{4}} .
$$

Combining these, we obtain

$$
\begin{aligned}
& \frac{u_{B}^{\prime}\left(c^{B}\left(s_{1}\right)\right)\left(\pi\left(s_{1}\right)+\pi\left(s_{3}\right)\right)}{u_{B}^{\prime}\left(c^{B}\left(s_{2}\right)\right)\left(\pi\left(s_{2}\right)+\pi\left(s_{4}\right)\right)}=\frac{p_{1}}{p_{2}} \frac{1+\frac{p_{3}}{p_{1}}}{1+\frac{p_{4}}{p_{2}}}=\frac{u_{A}^{\prime}\left(c^{A}\left(s_{1}\right)\right) \pi\left(s_{1}\right)}{u_{A}^{\prime}\left(c^{A}\left(s_{2}\right)\right) \pi\left(s_{2}\right)} \frac{1+\frac{u_{A}^{\prime}\left(c^{A}\left(s_{3}\right)\right) \pi\left(s_{3}\right)}{u_{A}^{\prime}\left(c^{A}\left(s_{1}\right)\right) \pi\left(s_{1}\right)}}{1+\frac{u_{A}^{\prime}\left(c^{A}\left(s_{4}\right)\right) \pi\left(s_{4}\right)}{u_{A}^{\prime}\left(c^{A}\left(s_{2}\right)\right) \pi\left(s_{2}\right)}}, \\
& \frac{u_{B}^{\prime}\left(c^{B}\left(s_{1}\right)\right)\left(\pi\left(s_{1}\right)+\pi\left(s_{3}\right)\right)}{u_{B}^{\prime}\left(c^{B}\left(s_{2}\right)\right)\left(\pi\left(s_{2}\right)+\pi\left(s_{4}\right)\right)}=\frac{u_{A}^{\prime}\left(c^{A}\left(s_{1}\right)\right) \pi\left(s_{1}\right)+u_{A}^{\prime}\left(c^{A}\left(s_{3}\right)\right) \pi\left(s_{3}\right)}{u_{A}^{\prime}\left(c^{A}\left(s_{2}\right)\right) \pi\left(s_{2}\right)+u_{A}^{\prime}\left(c^{A}\left(s_{4}\right)\right) \pi\left(s_{4}\right)} .
\end{aligned}
$$

Indeed, in a manner of contradiction, assume that $c^{B}\left(s_{1}\right)=c^{B}\left(s_{2}\right)$ and note that this implies

$$
\begin{aligned}
\frac{u_{B}^{\prime}\left(c^{B}\left(s_{1}\right)\right)\left(\pi\left(s_{1}\right)+\pi\left(s_{3}\right)\right)}{u_{B}^{\prime}\left(c^{B}\left(s_{2}\right)\right)\left(\pi\left(s_{2}\right)+\pi\left(s_{4}\right)\right)} & =\frac{\left(\pi\left(s_{1}\right)+\pi\left(s_{3}\right)\right)}{\left(\pi\left(s_{2}\right)+\pi\left(s_{4}\right)\right)}=\frac{u_{A}^{\prime}\left(c^{A}\left(s_{1}\right)\right) \pi\left(s_{1}\right)+u_{A}^{\prime}\left(c^{A}\left(s_{3}\right)\right) \pi\left(s_{3}\right)}{u_{A}^{\prime}\left(c^{A}\left(s_{2}\right)\right) \pi\left(s_{2}\right)+u_{A}^{\prime}\left(c^{A}\left(s_{4}\right)\right) \pi\left(s_{4}\right)} \\
& =\frac{u_{A}^{\prime}\left(3-c^{B}\left(s_{1}\right)\right) \pi\left(s_{1}\right)+u_{A}^{\prime}\left(2-c^{B}\left(s_{1}\right)\right) \pi\left(s_{3}\right)}{u_{A}^{\prime}\left(3-c^{B}\left(s_{1}\right)\right) \pi\left(s_{2}\right)+u_{A}^{\prime}\left(4-c^{B}\left(s_{1}\right)\right) \pi\left(s_{4}\right)} \\
& >1,
\end{aligned}
$$


since

$$
\frac{u_{A}^{\prime}\left(3-c^{B}\left(s_{1}\right)\right) \pi\left(s_{1}\right)+u_{A}^{\prime}\left(2-c^{B}\left(s_{1}\right)\right) \pi\left(s_{3}\right)}{u_{A}^{\prime}\left(3-c^{B}\left(s_{1}\right)\right) \pi\left(s_{2}\right)+u_{A}^{\prime}\left(4-c^{B}\left(s_{1}\right)\right) \pi\left(s_{4}\right)}>\frac{\left(\pi\left(s_{1}\right)+\pi\left(s_{3}\right)\right)}{\left(\pi\left(s_{2}\right)+\pi\left(s_{4}\right)\right)}
$$

is equivalent to

$$
\begin{aligned}
& u_{A}^{\prime}\left(3-c^{B}\left(s_{1}\right)\right) \pi\left(s_{1}\right)\left(\pi\left(s_{2}\right)+\pi\left(s_{4}\right)\right)+u_{A}^{\prime}\left(2-c^{B}\left(s_{1}\right)\right) \pi\left(s_{3}\right)\left(\pi\left(s_{2}\right)+\pi\left(s_{4}\right)\right) \\
& \quad>u_{A}^{\prime}\left(3-c^{B}\left(s_{1}\right)\right) \pi\left(s_{2}\right)\left(\pi\left(s_{1}\right)+\pi\left(s_{3}\right)\right)+u_{A}^{\prime}\left(4-c^{B}\left(s_{1}\right)\right) \pi\left(s_{4}\right)\left(\pi\left(s_{1}\right)+\pi\left(s_{3}\right)\right), \\
& \quad\left[u_{A}^{\prime}\left(3-c^{B}\left(s_{1}\right)\right)-u_{A}^{\prime}\left(4-c^{B}\left(s_{1}\right)\right)\right] \pi\left(s_{1}\right) \pi\left(s_{4}\right) \\
& \quad+\left[u_{A}^{\prime}\left(2-c^{B}\left(s_{1}\right)\right)-u_{A}^{\prime}\left(4-c^{B}\left(s_{1}\right)\right)\right] \pi\left(s_{3}\right) \pi\left(s_{4}\right) \\
& \quad+\left[u_{A}^{\prime}\left(2-c^{B}\left(s_{1}\right)\right)-u_{A}^{\prime}\left(3-c^{B}\left(s_{1}\right)\right)\right] \pi\left(s_{2}\right) \pi\left(s_{3}\right)>0,
\end{aligned}
$$

which is always satisfied, since $u_{A}^{\prime}$ is a decreasing function. We thus obtain a contradiction to the assumption that $B$ is fully insured against idiosyncratic risk in equilibrium. Q.E.D.

Claim 2: . If condition (3) holds, A's expected consumption is higher than her expected initial endowment.

PROOF: From the fact that $B$ 's utility function is concave and, thus, $B$ partially insures against risk, it follows that the equilibrium consumption of $A$ satisfies $c^{A}\left(s_{1}\right)<2, c^{A}\left(s_{2}\right)>$ $1, c^{A}\left(s_{3}\right)<1$, and $c^{A}\left(s_{4}\right)>2$ with

$$
\begin{aligned}
c^{A}\left(s_{4}\right) & =4-c^{B}\left(s_{2}\right)>c^{A}\left(s_{1}\right)=3-c^{B}\left(s_{1}\right) \\
& >c^{A}\left(s_{2}\right)=3-c^{B}\left(s_{2}\right)>c^{A}\left(s_{3}\right)=2-c^{B}\left(s_{1}\right) .
\end{aligned}
$$

From $A$ 's f.o.c. we then conclude that the equilibrium prices satisfy

$$
\frac{p_{4}^{*}}{\pi\left(s_{4}\right)}<\frac{p_{1}^{*}}{\pi\left(s_{1}\right)}<\frac{p_{2}^{*}}{\pi\left(s_{2}\right)}<\frac{p_{3}^{*}}{\pi\left(s_{3}\right)} .
$$

Suppose to the contrary of Claim 2 that

$$
E_{\pi}\left[c^{A}(s)\right]<E_{\pi}\left(e^{A}(s)\right)=2 \pi\left(s_{1}\right)+\pi\left(s_{2}\right)+\pi\left(s_{3}\right)+2 \pi\left(s_{4}\right)
$$

and, hence,

$$
\begin{aligned}
& {\left[3-c^{B}\left(s_{1}\right)\right] \pi\left(s_{1}\right)+\left[2-c^{B}\left(s_{1}\right)\right] \pi\left(s_{3}\right)+\left[3-c^{B}\left(s_{2}\right)\right] \pi\left(s_{2}\right)} \\
& \quad+\left[4-c^{B}\left(s_{2}\right)\right] \pi\left(s_{4}\right) \leq 2 \pi\left(s_{1}\right)+\pi\left(s_{2}\right)+\pi\left(s_{3}\right)+2 \pi\left(s_{4}\right)
\end{aligned}
$$

or

$$
c^{B}\left(s_{2}\right) \geq 2+\frac{\pi\left(s_{1}\right)+\pi\left(s_{3}\right)}{\pi\left(s_{2}\right)+\pi\left(s_{4}\right)}\left[1-c^{B}\left(s_{1}\right)\right] .
$$

It follows that

$$
\begin{aligned}
& E_{\pi} u_{A}\left(c^{A}\right) \\
& \quad=\pi\left(s_{1}\right) u_{A}\left(3-c^{B}\left(s_{1}\right)\right)+\pi\left(s_{3}\right) u_{A}\left(2-c^{B}\left(s_{1}\right)\right)+\pi\left(s_{2}\right) u_{A}\left(3-c^{B}\left(s_{2}\right)\right)
\end{aligned}
$$




$$
\begin{aligned}
& +\pi\left(s_{4}\right) u_{A}\left(4-c^{B}\left(s_{2}\right)\right) \leq \pi\left(s_{1}\right) u_{A}\left(2+\left(1-c^{B}\left(s_{1}\right)\right)\right)+\pi\left(s_{3}\right) u_{A}\left(1+\left(1-c^{B}\left(s_{1}\right)\right)\right) \\
& +\pi\left(s_{2}\right) u_{A}\left(1-\frac{\pi\left(s_{1}\right)+\pi\left(s_{3}\right)}{\pi\left(s_{2}\right)+\pi\left(s_{4}\right)}\left(1-c^{B}\left(s_{1}\right)\right)\right) \\
& +\pi\left(s_{4}\right) u_{A}\left(2-\frac{\pi\left(s_{1}\right)+\pi\left(s_{3}\right)}{\pi\left(s_{2}\right)+\pi\left(s_{4}\right)}\left(1-c^{B}\left(s_{1}\right)\right)\right) \\
& <\left(\pi\left(s_{1}\right)+\pi\left(s_{4}\right)\right) u_{A}\left(2+\left(1-c^{B}\left(s_{1}\right)\right) \frac{\pi\left(s_{1}\right) \pi\left(s_{2}\right)-\pi\left(s_{3}\right) \pi\left(s_{4}\right)}{\left(\pi\left(s_{2}\right)+\pi\left(s_{4}\right)\right)\left(\pi\left(s_{1}\right)+\pi\left(s_{4}\right)\right)}\right) \\
& +\left(\pi\left(s_{2}\right)+\pi\left(s_{3}\right)\right) u_{A}\left(1+\frac{\pi\left(s_{3}\right) \pi\left(s_{4}\right)-\pi\left(s_{1}\right) \pi\left(s_{2}\right)}{\left(\pi\left(s_{2}\right)+\pi\left(s_{4}\right)\right)\left(\pi\left(s_{2}\right)+\pi\left(s_{3}\right)\right)}\left(1-c^{B}\left(s_{1}\right)\right)\right) .
\end{aligned}
$$

Since $c^{B}\left(s_{1}\right)>1$ for $\pi\left(s_{1}\right) \pi\left(s_{2}\right)-\pi\left(s_{3}\right) \pi\left(s_{4}\right) \leq 0$, this is a mean-preserving spread of the initial endowment and we have $E_{\pi} u_{A}\left(c^{A}\right)<E_{\pi} u_{A}\left(e^{A}\right)$, in contradiction to utility maximization.

\section{Derivations for Example 2}

ClAIM 3: It is impossible to ensure all agents in the economy against idiosyncratic risk.

Proof: Suppose to the contrary that $c^{i}\left(s_{1}\right)=c^{i}\left(s_{2}\right)$ for all agents $i \in\{A ; B ; C ; D\}$. For $A$ to be fully insured across $s_{1}$ and $s_{2}$, we need

$$
\frac{u_{A}^{\prime}\left(c^{A}\left(s_{1}\right)\right)}{u_{A}^{\prime}\left(c^{A}\left(s_{2}\right)\right)}=1=\frac{p_{1}}{p_{2}} .
$$

Furthermore, since $C$ and $D$ are fully insured across $s_{1}$ and $s_{2}$, the measurability requirement on their consumption implies that they are fully insured across all states,

$$
c^{i}\left(s_{1}\right)=c^{i}\left(s_{2}\right)=c^{i}\left(s_{3}\right)=c^{i}\left(s_{4}\right), \quad i \in\{C ; B\},
$$

and, hence, $\frac{u_{B}^{\prime}\left(c^{B}\left(s_{3}\right)\right)}{u_{B}^{\prime}\left(c^{B}\left(s_{4}\right)\right)}=\frac{p_{1}+p_{3}}{p_{2}+p_{4}}$ or $p_{3}=p_{4}$. But this would imply that $\frac{u_{i}^{\prime}\left(c^{i}\left(s_{3}\right)\right)}{u_{i}^{\prime}\left(c^{i}\left(s_{4}\right)\right)}=\frac{p_{3}}{p_{4}}=1$ or that both $A$ and $D$ have to be fully insured across states $s_{3}$ and $s_{4}$, which is impossible. Q.E.D.

Claim 4: In general, $A$ and $D$ will not be fully insured against idiosyncratic risk in equilibrium.

Proof: To give an example, suppose that $u_{i}(c)=\ln c$ for $i \in\{A ; B ; D\}$, whereas $u_{C}(c)=c^{\frac{1}{2}}$. Suppose that $A$ and $D$ were fully insured against idiosyncratic risk. Then by the same argument as above, $p_{1}=p_{2}=p$. Since $B$ and $C$ are risk averse, they will try to smooth consumption across the states they perceive. Hence, in equilibrium, their consumption will satisfy

$$
\begin{aligned}
& 2>c^{B}\left(s_{2}\right)=c^{B}\left(s_{4}\right)>c^{B}\left(s_{1}\right)=c^{B}\left(s_{3}\right)>1, \\
& 2>c^{C}\left(s_{1}\right)=c^{C}\left(s_{4}\right)>c^{C}\left(s_{2}\right)=c^{C}\left(s_{3}\right)>1 .
\end{aligned}
$$

But then

$$
c^{A}\left(s_{1}\right)=\frac{6-c^{B}\left(s_{1}\right)-c^{C}\left(s_{1}\right)-c^{D}\left(s_{1}\right)}{2}=\frac{6-c^{B}\left(s_{2}\right)-c^{C}\left(s_{2}\right)-c^{D}\left(s_{1}\right)}{2}=c^{A}\left(s_{2}\right)
$$


and we obtain

$$
c^{B}\left(s_{1}\right)+c^{C}\left(s_{1}\right)=c^{B}\left(s_{2}\right)+c^{C}\left(s_{2}\right) .
$$

The demand functions of $B$ and $C$ satisfy

$$
\begin{aligned}
& c^{B}\left(s_{1}\right)=c^{B}\left(s_{3}\right)=\frac{3 p+p_{3}+2 p_{4}}{2\left(p_{3}+p\right)}, \\
& c^{B}\left(s_{2}\right)=c^{B}\left(s_{4}\right)=\frac{3 p+p_{3}+2 p_{4}}{2\left(p_{4}+p\right)}, \\
& c^{C}\left(s_{2}\right)=c^{C}\left(s_{3}\right)=\frac{3 p+p_{3}+2 p_{4}}{2 p+p_{3}+p_{4}} \frac{\left(p+p_{4}\right)}{\left(p+p_{3}\right)}, \\
& c^{C}\left(s_{1}\right)=c^{C}\left(s_{4}\right)=\frac{3 p+p_{3}+2 p_{4}}{2 p+p_{3}+p_{4}} \frac{\left(p+p_{3}\right)}{\left(p+p_{4}\right)},
\end{aligned}
$$

and substituting into (8), we obtain

$$
\frac{3 p+p_{3}+2 p_{4}}{2\left(p_{3}+p\right)}+\frac{3 p+p_{3}+2 p_{4}}{2 p+p_{3}+p_{4}} \frac{\left(p+p_{3}\right)}{\left(p+p_{4}\right)}=\frac{3 p+p_{3}+2 p_{4}}{2 p+p_{3}+p_{4}} \frac{\left(p+p_{4}\right)}{\left(p+p_{3}\right)}+\frac{3 p+p_{3}+2 p_{4}}{2\left(p_{4}+p\right)} .
$$

Without loss of generality, we can normalize $3 p+p_{3}+2 p_{4}=1$ and simplify to

$$
2 p\left(p_{3}-p_{4}\right)=\left(p_{4}-p_{3}\right)\left(p_{4}+p_{3}\right) .
$$

Since the only solution of this equation is $p_{3}=p_{4}$, it follows that

$$
\begin{aligned}
& c^{A}\left(s_{3}\right)=c^{A}\left(s^{4}\right), \\
& c^{D}\left(s_{3}\right)=c^{D}\left(s^{4}\right), \\
& c^{B}\left(s_{3}\right)=c^{B}\left(s_{4}\right) \\
& c^{C}\left(s_{3}\right)=c^{C}\left(s_{4}\right),
\end{aligned}
$$

in contradiction to the existence of aggregate risk.

Q.E.D.

To simplify the proofs of the following results, we state and prove the following lemma.

LEMMA 3: Consider two agents $i$ and $j$ such that $j$ is weakly less constrained than $i$, that is, $A^{i} \subseteq A^{j}$ and $\Omega^{i}$ is weakly coarser than $\Omega^{j}$. In equilibrium, $\pi$-a.s. for any path $\omega^{i} \in \Omega^{i}$,

$$
\begin{aligned}
& \lim _{T \rightarrow \infty} \frac{1}{T+1} \ln \frac{u_{i}^{\prime}\left(c^{i}\left(\omega_{T+1}^{i}\right)\right)}{\sum_{\tilde{\omega}_{T+1}^{j} \subseteq \omega_{T+1}^{i}} u_{j}^{\prime}\left(c^{j}\left(\tilde{\omega}_{T+1}^{j}\right)\right) \pi^{j}\left(\tilde{\omega}_{T+1}^{j} \mid \omega_{T+1}^{i}\right)} \\
& \quad=\ln \frac{\beta_{j}}{\beta_{i}}+\sum_{w^{i} \in W^{i}} \pi\left(w^{i}\right) \ln \frac{\pi\left(w^{i}\right)}{\pi^{i}\left(w^{i}\right)}-\sum_{w^{i} \in W^{i}} \pi\left(w^{i}\right) \ln \frac{\pi\left(w^{i}\right)}{\pi^{j}\left(w^{i}\right)}
\end{aligned}
$$


and

$$
\begin{gathered}
\lim _{T \rightarrow \infty} \frac{1}{T+1} \ln \frac{\sum_{\tilde{\omega}_{T+1}^{j} \subseteq \omega_{T+1}^{i}} u_{j}^{\prime}\left(c^{j}\left(\tilde{\omega}_{T+1}^{j}\right)\right) \pi^{j}\left(\tilde{\omega}_{T+1}^{j} \mid \omega_{T+1}^{i}\right)}{u_{i}^{\prime}\left(c^{i}\left(\omega_{T+1}^{i}\right)\right)} \\
=\ln \frac{\beta_{i}}{\beta_{j}}+\sum_{w^{i} \in W^{i}} \pi\left(w^{i}\right) \ln \frac{\pi\left(w^{i}\right)}{\pi^{j}\left(w^{i}\right)}-\sum_{w^{i} \in W^{i}} \pi\left(w^{i}\right) \ln \frac{\pi\left(w^{i}\right)}{\pi^{i}\left(w^{i}\right)} .
\end{gathered}
$$

If, furthermore, $\pi^{i}\left(w^{i}\right)=\pi^{j}\left(w^{i}\right)$ for all $w^{i} \in W^{i}$ and $\beta^{i}=\beta^{j}$, then for every $\omega_{T+1}^{i} \in \Omega^{i}$,

$$
\frac{u_{i}^{\prime}\left(c^{i}\left(\omega_{T+1}^{i}\right)\right)}{\sum_{\tilde{\omega}_{T+1}^{j} \subseteq \omega_{T+1}^{i}} u_{j}^{\prime}\left(c^{j}\left(\tilde{\omega}_{T+1}^{j}\right)\right) \pi^{j}\left(\tilde{\omega}_{T+1}^{j} \mid \omega_{T+1}^{i}\right)}=\frac{u_{i}^{\prime}\left(c^{i}\left(\sigma_{0}\right)\right)}{u_{j}^{\prime}\left(c^{j}\left(\sigma_{0}\right)\right)} .
$$

Proof: We will use the analogue of the Blume and Easley (2006) decomposition. Applying condition (2) to $i$ and $j$,

$$
\frac{u_{i}^{\prime}\left(c^{i}\left(\sigma_{0}\right)\right)}{\beta_{i} u_{i}^{\prime}\left(c^{i}\left(\omega_{T+1}^{i}\right)\right) \pi^{i}\left(\omega_{T+1}^{i}\right)}=\frac{u_{j}^{\prime}\left(c^{j}\left(\sigma_{0}\right)\right)}{\beta_{j} \sum_{\tilde{\omega}_{T+1}^{j} \subseteq \omega_{T+1}^{i}} u_{j}^{\prime}\left(c^{j}\left(\tilde{\omega}_{T+1}^{j}\right)\right) \pi^{j}\left(\tilde{\omega}_{T+1}^{j}\right)} .
$$

Hence,

$$
\frac{u_{i}^{\prime}\left(c^{i}\left(\sigma_{0}\right)\right)}{\beta_{i} u_{i}^{\prime}\left(c^{i}\left(\omega_{T+1}^{i}\right)\right) \pi^{i}\left(\omega_{T+1}^{i}\right)}=\frac{u_{j}^{\prime}\left(c^{j}\left(\sigma_{0}\right)\right)}{\beta_{j} \pi^{j}\left(\omega_{T+1}^{i}\right) \sum_{\tilde{\omega}_{T+1}^{j} \subseteq \omega_{T+1}^{i}} u_{j}^{\prime}\left(c^{j}\left(\tilde{\omega}_{T+1}^{j}\right)\right) \pi^{j}\left(\tilde{\omega}_{T+1}^{j} \mid \omega_{T+1}^{i}\right)},
$$

which reduces to

$$
\begin{aligned}
\frac{u_{i}^{\prime}\left(c^{i}\left(\omega_{T+1}^{i}\right)\right)}{\sum_{\tilde{\omega}_{T+1}^{j} \subseteq \omega_{T+1}^{i}} u_{j}^{\prime}\left(c^{j}\left(\tilde{\omega}_{T+1}^{j}\right)\right) \pi^{j}\left(\tilde{\omega}_{T+1}^{j} \mid \omega_{T+1}^{i}\right)} & =\frac{\beta_{j}}{\beta_{i}} \frac{\pi^{j}\left(\omega_{T+1}^{i}\right)}{\pi^{i}\left(\omega_{T+1}^{i}\right)} \frac{u_{i}^{\prime}\left(c^{i}\left(\sigma_{0}\right)\right)}{u_{j}^{\prime}\left(c^{j}\left(\sigma_{0}\right)\right)} \\
& =\frac{u_{i}^{\prime}\left(c^{i}\left(\sigma_{0}\right)\right)}{u_{j}^{\prime}\left(c^{j}\left(\sigma_{0}\right)\right)} \prod_{t=1}^{T+1} \frac{\beta_{j}}{\beta_{i}} \frac{\pi^{j}\left(w_{t}^{i}\right)}{\pi^{i}\left(w_{t}^{i}\right)} .
\end{aligned}
$$

If $\pi^{i}\left(w^{i}\right)=\pi^{j}\left(w^{i}\right)$ for all $w^{i} \in W^{i}$ and $\beta^{i}=\beta^{j},(11)$ immediately obtains. Otherwise,

$$
\begin{aligned}
& \lim _{T \rightarrow \infty} \frac{1}{T+1} \ln \frac{u_{i}^{\prime}\left(c^{i}\left(\omega_{T+1}^{i}\right)\right)}{\sum_{\tilde{\omega}_{T+1}^{j} \subseteq \omega_{T+1}^{i}} u_{j}^{\prime}\left(c^{j}\left(\tilde{\omega}_{T+1}^{j}\right)\right) \pi^{j}\left(\tilde{\omega}_{T+1}^{j} \mid \omega_{T+1}^{i}\right)} \\
& \quad=\lim _{T \rightarrow \infty} \ln \frac{\beta_{j}}{\beta_{i}}+\lim _{T \rightarrow \infty} \frac{1}{T+1} \sum_{t=1}^{T+1} \ln \frac{\pi^{j}\left(w_{t}^{i}\right)}{\pi^{i}\left(w_{t}^{i}\right)}+\lim _{T \rightarrow \infty} \frac{1}{T+1} \ln \frac{u_{i}^{\prime}\left(c^{i}\left(\sigma_{0}\right)\right)}{u_{j}^{\prime}\left(c^{j}\left(\sigma_{0}\right)\right)} .
\end{aligned}
$$


Since $u_{i}^{\prime}\left(c^{i}\left(\sigma_{0}\right)\right)$ and $u_{j}^{\prime}\left(c^{j}\left(\sigma_{0}\right)\right)$ are finite, the third term on the right-hand side (r.h.s.) converges to 0 . Furthermore,

$$
\begin{aligned}
& \lim _{T \rightarrow \infty} \frac{1}{T+1} \ln \frac{u_{i}^{\prime}\left(c^{i}\left(\omega_{T+1}^{i}\right)\right)}{\sum_{\tilde{\omega}_{T+1}^{j} \subseteq \omega_{T+1}^{i}} u_{j}^{\prime}\left(c^{j}\left(\tilde{\omega}_{T+1}^{j}\right)\right) \pi^{j}\left(\tilde{\omega}_{T+1}^{j} \mid \omega_{T+1}^{i}\right)} \\
& =\lim _{T \rightarrow \infty} \ln \frac{\beta_{j}}{\beta_{i}}+\lim _{T \rightarrow \infty} \frac{1}{T+1} \sum_{t=1}^{T+1}\left(\ln \pi^{j}\left(w_{t}^{i}\right)-\ln \pi\left(w_{t}^{i}\right)\right) \\
& \quad+\lim _{T \rightarrow \infty} \frac{1}{T+1} \sum_{t=1}^{T+1}\left(\ln \pi\left(w_{t}^{i}\right)-\ln \pi^{i}\left(w_{t}^{i}\right)\right) .
\end{aligned}
$$

Since $\ln \frac{\pi\left(w_{t}^{i}\right)}{\pi^{i}\left(w_{t}^{i}\right)}$ and $\ln \frac{\pi\left(w_{t}^{i}\right)}{\pi^{j}\left(w_{t}^{i}\right)}$ are i.i.d and are equal in expectations to the relative entropy of $i$ 's and $j$ 's beliefs with respect to the truth $\pi$, we obtain that (9) $\pi$-a.s. holds. The derivations for (10) are analogous and, thus, are omitted.

Q.E.D.

\section{Proof of Proposition 2}

Using Lemma 3 for the case $\Omega^{i}=\Omega^{j}$, we obtain that for two such agents, $i$ and $j$, with $\ln \frac{\beta_{i}}{\beta_{j}}+\left(\sum_{w^{i} \in W^{i}} \pi\left(w^{i}\right) \ln \frac{\pi\left(w^{i}\right)}{\pi^{j}\left(w^{i}\right)}-\sum_{w^{i} \in W^{i}} \pi\left(w^{i}\right) \ln \frac{\pi\left(w^{i}\right)}{\pi^{i}\left(w^{i}\right)}\right)>0, \pi$-a.s. on every $\omega^{i} \in \Omega^{i}$, $\lim _{T \rightarrow \infty} \frac{1}{T+1} \ln \frac{u_{j}^{\prime}\left(c^{j}\left(\omega_{T+1}^{i}\right)\right)}{u_{i}^{\prime}\left(c^{i}\left(\omega_{T+1}^{i}\right)\right)}=\ln \frac{\beta_{i}}{\beta_{j}}+\left(\sum_{w^{i} \in W^{i}} \pi\left(w^{i}\right) \ln \frac{\pi\left(w^{i}\right)}{\pi^{j}\left(w^{i}\right)}-\sum_{w^{i} \in W^{i}} \pi\left(w^{i}\right) \ln \frac{\pi\left(w^{i}\right)}{\pi^{i}\left(w^{i}\right)}\right)>0$.

Since $u_{i}^{\prime}\left(c^{i}\left(\omega_{T+1}\right)\right)>u_{i}^{\prime}\left(m^{\prime}\right)$ for all $\omega_{T+1}^{i}$, the denominator in the $\ln$ on the left-hand side (l.h.s.) is bounded. If the numerator $u_{j}^{\prime}\left(c^{j}\left(\omega_{T+1}\right)\right)$ were also bounded, the 1.h.s. would converge to 0 in contradiction to the equality above. Hence, $\pi$-a.s., $u_{j}^{\prime}\left(c^{j}\left(\omega_{T+1}\right)\right) \rightarrow \infty$ and, therefore, $c^{j}\left(\omega_{T+1}\right) \rightarrow 0$ or $j$ vanishes.

\section{Proof of Proposition 3}

Order the agents from 1 to $n$ such that $A^{1} \supseteq A^{2} \supseteq \ldots A^{n}$ and, thus, $\Omega^{1}$ finer than $\Omega^{2}$ finer than... $\Omega^{n}$. By measurability of initial endowment, w.l.o.g., set $\Omega^{1}=\Omega$.

LemMa 4: Agent 1, cannot be the only one to survive on a path $\sigma \in \Omega$.

PROOF: Take a path $\sigma$ and assume that $\lim _{t \rightarrow \infty} c^{i}\left(\sigma_{t}\right)=0$ for all $i>1$. Measurability of consumption implies for all $i>1$ and $\omega^{i} \in \Omega^{i}$ such that $\sigma \in \omega^{i}, c^{i}\left(\sigma_{t}\right)=c^{i}\left(\sigma_{t}^{\prime}\right)$ for all $\sigma_{t}^{\prime} \in \omega_{t}^{i}$ and, thus, $\lim _{t \rightarrow \infty} c^{i}\left(\sigma_{t}^{\prime}\right)=0$. Since $\Omega^{2}$ is the finest partition among those of agents $i>1$, there is a $t\left(\omega^{2}\right)$ such that $\sigma \in \omega^{2}$ with $c^{i}\left(\sigma_{t}\right)<\varepsilon<\frac{m}{n-1}$ for all $i>1$ and all $t \geq t\left(\omega^{2}\right)$, implying $c^{1}\left(\tilde{\sigma}_{t}\right) \geq m-(n-1) \varepsilon>0$ for all $\tilde{\sigma} \in \omega^{2}$ and $t \geq t\left(\omega^{2}\right)$. By (12), and since discount factors and beliefs are identical, we can use (11), replacing $i$ by 2 and $j$ by 1 , where $\frac{u_{2}^{\prime}\left(c^{2}\left(\sigma_{0}\right)\right)}{u_{1}^{\prime}\left(c^{1}\left(\sigma_{0}\right)\right)}<\infty$. We have $\sum_{\tilde{\sigma}_{T+1} \in \omega_{T+1}^{2}} u_{1}^{\prime}\left(c^{1}\left(\tilde{\sigma}_{T+1}\right)\right) \pi^{1}\left(\tilde{\sigma}_{T+1} \mid \omega_{T+1}^{2}\right) \leq u_{1}^{\prime}(m-(n-$ 1) $\varepsilon)<\infty$ for all $T+1>t\left(\omega^{2}\right)$. However, $\lim _{t \rightarrow \infty} c^{2}\left(\sigma_{t}\right)=0$ and, thus, by Assumption 1 , $\lim _{T+1 \rightarrow \infty} u_{2}^{\prime}\left(c^{2}\left(\omega_{T+1}^{2}\right)\right)=\infty$, in contradiction to (11). 
It follows that at least one agent, $i>1$, has to survive on $\sigma$ and, thus, on $\omega^{i}$ such that $\sigma \in \omega^{i}$.

LEMMA 5: For any $\omega_{t}^{n} \in \Omega^{n}$,

$$
\lim _{t \rightarrow \infty} \sup \sum_{\tilde{\sigma}_{t} \in \omega_{t}^{n}} u_{1}^{\prime}\left(c^{1}\left(\tilde{\sigma}_{t}\right)\right) \pi^{1}\left(\tilde{\sigma}_{t} \mid \omega_{t}^{n}\right) \leq \max _{i \in\{1 \ldots n\}} u_{i}^{\prime}\left(\frac{m}{n}\right) \frac{u_{1}^{\prime}\left(c^{1}\left(\sigma_{0}\right)\right)}{u_{i}^{\prime}\left(c^{i}\left(\sigma_{0}\right)\right)} .
$$

Proof: Take a path $\sigma \in \Omega$ and note that for every $\sigma_{t}$, there is an $i\left(\sigma_{t}\right) \in I$ such that $c_{i}\left(\sigma_{t}\right) \geq \frac{m}{n}$. Since discount factors and beliefs are identical, it follows that for any $\sigma_{t} \in$ $\Omega$ and the corresponding $i\left(\sigma_{t}\right)$ and $\omega^{i\left(\sigma_{t}\right)} \in \Omega^{i\left(\sigma_{t}\right)}$ such that $\sigma \in \omega^{i\left(\sigma_{t}\right)}$, we can use (11), replacing $i$ by $i\left(\sigma_{t}\right)$ and $j$ by 1 to obtain

$$
\sum_{\tilde{\sigma}_{t} \in \omega_{t}^{i\left(\sigma_{t}\right)}} u_{1}^{\prime}\left(c^{1}\left(\tilde{\sigma}_{t}\right)\right) \pi^{1}\left(\tilde{\sigma}_{t} \mid \omega_{t}^{i\left(\sigma_{t}\right)}\right) \leq u_{i\left(\sigma_{t}\right)}^{\prime}\left(\frac{m}{n}\right) \frac{u_{1}^{\prime}\left(c^{1}\left(\sigma_{0}\right)\right)}{u_{i\left(\sigma_{t}\right)}^{\prime}\left(c^{i\left(\sigma_{t}\right)}\left(\sigma_{0}\right)\right)} .
$$

It follows that for any $\sigma_{t}$ and $\omega_{t}^{i\left(\sigma_{t}\right)} \in \Omega^{i\left(\sigma_{t}\right)}$,

$$
\sum_{\tilde{\sigma}_{t} \in \omega_{t}^{i\left(\sigma_{t}\right)}} u_{1}^{\prime}\left(c^{1}\left(\tilde{\sigma}_{t}\right)\right) \pi^{1}\left(\tilde{\sigma}_{t} \mid \omega_{t}^{i\left(\sigma_{t}\right)}\right) \leq \max _{i \in\{1 \ldots n\}} u_{i}^{\prime}\left(\frac{m}{n}\right) \frac{u_{1}^{\prime}\left(c^{1}\left(\sigma_{0}\right)\right)}{u_{i}^{\prime}\left(c^{i}\left(\sigma_{0}\right)\right)}=P .
$$

Consider $\omega_{t}^{n} \in \Omega^{n}$ and note that we can partition $\omega_{t}^{n}$ into at most $n-1$ subsets $x_{k}\left(\omega_{t}^{n}\right) \subseteq \Omega_{t}$ such that on $\sigma_{t} \in x_{k}\left(\omega_{t}^{n}\right)$, agent $k$ is the agent with the coarsest partition and consumption $c^{k}\left(\sigma_{t}\right) \geq \frac{m}{n}$. It follows that for every $\omega_{t}^{k} \subseteq x_{k}\left(\omega_{t}^{n}\right)$,

$$
\begin{array}{r}
\sum_{\tilde{\sigma}_{t} \in \omega_{t}^{k}} u_{1}^{\prime}\left(c^{1}\left(\tilde{\sigma}_{t}\right)\right) \pi^{1}\left(\tilde{\sigma}_{t} \mid \omega_{t}^{k}\right) \leq P \quad \text { and thus, } \\
\sum_{\omega_{t}^{k} \subseteq x_{k}\left(\omega_{t}^{n}\right)} \pi^{1}\left(\omega_{t}^{k} \mid x_{k}\left(\omega_{t}^{n}\right)\right) \sum_{\tilde{\sigma}_{t} \in \omega_{t}^{k}} u_{1}^{\prime}\left(c^{1}\left(\tilde{\sigma}_{t}\right)\right) \pi^{1}\left(\tilde{\sigma}_{t} \mid \omega_{t}^{k}\right) \leq P .
\end{array}
$$

Finally, since the number of the elements of the partition $\left(x_{k}\left(\omega_{t}^{n}\right)\right)_{k \in\{1 \ldots n\}}$ is finite, we have

$$
\sum_{k=1}^{n} \pi^{1}\left(x_{k}\left(\omega_{t}^{n}\right) \mid \omega_{t}^{n}\right) \sum_{\tilde{\sigma}_{t} \in x_{k}\left(\omega_{t}^{n}\right)} u_{1}^{\prime}\left(c^{1}\left(\tilde{\sigma}_{t}\right)\right) \pi^{1}\left(\tilde{\sigma}_{t} \mid x_{k}\left(\omega_{t}^{n}\right)\right)=\sum_{\tilde{\sigma}_{t} \in \omega_{t}^{n}} u_{1}^{\prime}\left(c^{1}\left(\tilde{\sigma}_{t}\right)\right) \pi^{1}\left(\tilde{\sigma}_{t} \mid \omega_{t}^{n}\right) \leq P
$$

for any $\omega_{t}^{n} \in \Omega^{n}$ and, thus,

$$
\lim _{t \rightarrow \infty} \sup \sum_{\tilde{\sigma}_{t} \in \omega_{t}^{n}} u_{1}^{\prime}\left(c^{1}\left(\tilde{\sigma}_{t}\right)\right) \pi^{1}\left(\tilde{\sigma}_{t} \mid \omega_{t}^{n}\right) \leq P,
$$

proving the statement of the lemma.

Q.E.D.

LEMMA 6: On any $\omega^{n} \in \Omega^{n}$, agent $n$ survives. Furthermore, the consumption of agent $n$ on each path is uniformly bounded away from 0 . 


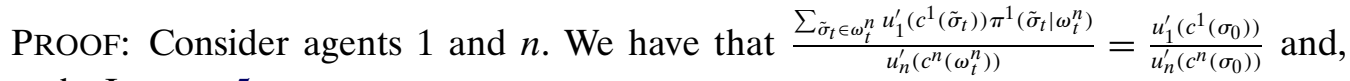
thus, by Lemma 5 ,

$$
\lim _{t \rightarrow \infty} \sup u_{n}^{\prime}\left(c^{n}\left(\omega_{t}^{n}\right)\right) \leq P \frac{u_{n}^{\prime}\left(c^{n}\left(\sigma_{0}\right)\right)}{u_{1}^{\prime}\left(c^{1}\left(\sigma_{0}\right)\right)} .
$$

Hence, $n$ survives on any $\omega_{t}^{n}$. Furthermore, (13) implies

$$
\lim _{t \rightarrow \infty} \inf c^{n}\left(\omega_{t}^{n}\right) \geq u_{n}^{\prime-1}\left(P \frac{u_{n}^{\prime}\left(c^{n}\left(\sigma_{0}\right)\right)}{u_{1}^{\prime}\left(c^{1}\left(\sigma_{0}\right)\right)}\right)>0 .
$$

LEMMA 7: For any $j \in\{2 \ldots n-1\}$ and any $\omega_{t}^{n} \in \Omega^{n}$

$$
\lim _{t \rightarrow \infty} \sup \sum_{\tilde{\omega}_{t}^{j} \leq \omega_{t}^{n}} u_{j}^{\prime}\left(c^{j}\left(\tilde{\omega}_{t}^{j}\right)\right) \pi^{j}\left(\tilde{\omega}_{t}^{j} \mid \omega_{t}^{n}\right) \leq P \frac{u_{j}^{\prime}\left(c^{j}\left(\sigma_{0}\right)\right)}{u_{1}^{\prime}\left(c^{1}\left(\sigma_{0}\right)\right)} .
$$

PROOF: The result follows from (11) in which $i$ is replaced by $n$ and $j$ remains $j$, and the fact that $u_{n}^{\prime}$ satisfies (13).

Q.E.D.

Lemma 8: Agent $j \in\{1 \ldots n-1\}$ survives $\pi$-a.s. on $\Omega$.

Proof: Suppose in a manner of contradiction that there exists a set $\tilde{\Omega}^{j} \subseteq \Omega^{j}, \pi\left(\tilde{\Omega}^{j}\right)>$ 0 and $\lim _{t \rightarrow \infty} c^{j}\left(\omega_{t}^{j}\right)=0 \pi$-a.s. $\omega^{j} \in \tilde{\Omega}^{j}$. Then $\lim _{t \rightarrow \infty} u_{j}^{\prime}\left(c^{j}\left(\tilde{\omega}_{t}^{j}\right)\right)=\infty \pi$-a.s. $\tilde{\Omega}^{j}$ and, hence, $\lim _{t \rightarrow \infty} \sum_{\tilde{\omega}_{t}^{j} \in \tilde{\Omega}^{j}} \pi\left(\tilde{\omega}_{t}^{j}\right) u_{j}^{\prime}\left(c^{j}\left(\tilde{\omega}_{t}^{j}\right)\right)=\infty$. Now let $\tilde{\Omega}^{n}$ denote the smallest measurable event on $\Omega^{n}$ such that for every $\omega^{j} \in \tilde{\Omega}^{j}$, there is an $\omega^{n} \in \tilde{\Omega}^{n}$ with $\omega^{j} \subseteq \omega^{n}$. Hence,

$$
\begin{aligned}
& \infty=\lim _{t \rightarrow \infty} \sum_{\tilde{\omega}_{t}^{j} \in \tilde{\Omega}^{j}} \pi\left(\tilde{\omega}_{t}^{j}\right) u_{j}^{\prime}\left(c^{j}\left(\tilde{\omega}_{t}^{j}\right)\right) \leq \lim _{t \rightarrow \infty} \sup \sum_{\substack{\omega_{t}^{n} \in \tilde{\Omega}^{n} \\
\tilde{n}_{t}}} \pi\left(\omega_{t}^{n}\right) \sum_{\substack{\tilde{\omega}_{t}^{j} \subseteq \omega_{t}^{n} \\
\tilde{\omega}_{t}^{j} \in \tilde{\Omega}^{j}}} \pi\left(\tilde{\omega}_{t}^{j} \mid \omega_{t}^{n}\right) u_{j}^{\prime}\left(c^{j}\left(\tilde{\omega}_{t}^{j}\right)\right) \\
& =\lim _{T \rightarrow \infty} \sup \sum_{\omega_{t}^{n} \in \tilde{\Omega}^{n}} \pi\left(\omega_{t}^{n}\right) \sum_{\substack{\tilde{\omega}_{t}^{j} \in \omega_{t}^{n} \\
\tilde{\omega}_{t} \in \tilde{\Omega}^{j}}} \pi^{j}\left(\tilde{\omega}_{t}^{j} \mid \omega_{t}^{n}\right) u_{j}^{\prime}\left(c^{j}\left(\tilde{\omega}_{t}^{j}\right)\right) \leq P \frac{u_{j}^{\prime}\left(c^{j}\left(\sigma_{0}\right)\right)}{u_{1}^{\prime}\left(c^{1}\left(\sigma_{0}\right)\right)}<\infty,
\end{aligned}
$$

where the first inequality follows from the definitions of $\tilde{\Omega}^{j}$ and $\tilde{\Omega}^{n}$, the second equality follows from the fact that $j$ 's beliefs are correct, and the two last inequalities from (14). We, thus, obtain a contradiction and conclude that $j \in\{1 \ldots n-1\} \pi$-a.s. survives on $\Omega^{j}$ and, thus, by measurability on $\Omega$.

Q.E.D.

\section{Proof of Proposition 4}

At the equilibrium prices, $i$ 's and $j$ 's optimization problems are given by (1). Endowments, discount factors, and utility functions coincide. Further, beliefs coincide on the common partition representing contingencies of which both can trade. Hence, the only difference between the two problems concerns the measurability requirements: $c^{j}$ has to be measurable relative to $\left(\Omega^{j} ; \mathcal{F}^{j}\right)$, whereas $c^{i}$ has to be measurable relative to the finer 
$\left(\Omega^{i} ; \mathcal{F}^{i}\right)$. Since $\Omega^{i}$ is coarser than $\Omega^{j}, i$ and $j$ are maximizing the same utility function at the same equilibrium prices and at the same initial endowment, but with $j$ having a strictly larger choice set w.r.t. inclusion than $i$. Hence, $V_{0}^{j}\left(c^{j}\right) \geq V_{0}^{i}\left(c^{i}\right)$ obtains in equilibrium.

\section{Proof of Proposition 5}

By Lemma 3, (9) obtains $\pi$-a.s. By assumption, the 1.h.s. of (9) is strictly positive. Since $u_{j}^{\prime}\left(c^{j}\left(\tilde{\omega}_{T+1}^{j}\right)\right) \geq u_{j}^{\prime}\left(m^{\prime}\right)$ for all $\tilde{\omega}_{T+1}^{j} \in \Omega^{j}$ and, thus, $\sum_{\tilde{\omega}_{T+1}^{j} \subseteq \omega_{T+1}^{i}} u_{j}^{\prime}\left(c^{j}\left(\tilde{\omega}_{T+1}^{j}\right)\right) \pi^{j}\left(\tilde{\omega}_{T+1}^{j} \mid\right.$ $\left.\omega_{T+1}^{i}\right) \geq u_{j}^{\prime}\left(m^{\prime}\right)$ for all $\omega_{T+1}^{i} \in \Omega^{i}$, this implies $u_{i}^{\prime}\left(c^{i}\left(\omega_{T+1}^{i}\right)\right) \rightarrow \infty$ or $c^{i}\left(\omega_{T+1}^{i}\right) \rightarrow 0, \pi$-a.s.

To prove Proposition 6, we first prove two special cases of it: Proposition 16, in which all agents have identical discount factors and identical, but potentially wrong beliefs, and Proposition 17, in which all agents have distinct beliefs and discount factors. Lemma 16 provides the bridge between these two cases, essentially establishing that when a set of agents has equal discount factors and beliefs, their total consumption in the limit coincides with the limit consumption of a single agent with those same discount factors and beliefs, and, furthermore, all of these agents a.s. survive.

PROPOSITION 16: Consider a population of agents with available sets of assets ordered with respect to inclusion $A^{1} \supset A^{2} \cdots \supset A^{n}$ and, thus, nested partitions, $\Omega^{1}$ strictly finer than $\Omega^{2} \ldots$ strictly finer than $\Omega^{n}$. If the constraints of any agent $i \geq 2$ are both relevant in the limit and relevant w.r.t. those of $i-1$, and if all agents have identical discount factors and identical (but not necessarily correct) beliefs, all agents a.s. survive.

PROOF: The proof of this proposition uses some of the lemmata derived in the proof of Proposition 3. In particular, note that the proofs of Lemmata 4, 5, and 6 only depend on the assumption that all agents have identical discount factors and identical, but not necessarily correct, beliefs. Hence, the results of these lemmata apply here.

Q.E.D.

LEMMA 9: Agent 1 survives a.s.

Proof: Since partitions are nested, for every $i, e^{i}$ is measurable w.r.t. $\Omega^{1}$, and so is the equilibrium consumption, $c^{i}$. Thus, w.l.o.g., we can set $\Omega^{1}=\Omega$ and conclude that agent 1 's constraints are irrelevant in the limit. Since those of agent 2 are both relevant and relevant w.r.t. those of 1, there are distinct $s$ and $s^{\prime} \in w^{2}$ for some $w^{2} \in W^{2}$ and $\epsilon>0$ such that for any $\sigma, \sigma^{\prime} \in \omega^{2}, \lim _{t \rightarrow \infty} \sup \left[e\left(\sigma_{t} ; s\right)-e\left(\sigma_{t}^{\prime} ; s^{\prime}\right)\right]>\epsilon$.

Since for every $j \geq 2, j$ 's consumption is measurable with respect to $\Omega^{2}$, for every $t$, every $\sigma_{t} \in \omega_{t}^{2}$, and $s, s^{\prime} \in w^{2}, \sum_{j \geq 2} c^{j}\left(\omega_{t}^{2} ; w^{2}\right) \leq e\left(\sigma_{t} ; s^{\prime}\right)$ and, hence, for every $\sigma \in \omega^{2}$ on which state $s$ occurs i.o. (which in turn implies that $w^{2}$ occurs i.o. on $\omega^{2}$ ),

$$
\begin{aligned}
& \lim _{T \rightarrow \infty} \sup c^{1}\left(\sigma_{T}\right) \\
& \quad=\lim _{T \rightarrow \infty} \sup \left[e\left(\sigma_{T}\right)-\sum_{j \geq 2} c^{j}\left(\sigma_{T}\right)\right] \\
& \quad \geq \lim _{T \rightarrow \infty} \sup \left[e\left(\sigma_{T}\right)-\min _{\tilde{s} \in w^{2}\left(\sigma_{T}\right)} e\left(\sigma_{T-1} ; \tilde{s}\right)\right] \geq \lim _{T \rightarrow \infty} \sup \left[e\left(\sigma_{T-1} ; s\right)-e\left(\sigma_{T-1} ; s^{\prime}\right)\right]>\epsilon .
\end{aligned}
$$

Since $s$ occurs i.o. a.s. w.r.t. $\pi^{i}$ for any $i$, it follows that $\pi$-a.s. and for any $i, \pi^{i}$-a.s., agent 1 survives on $\sigma$. Hence, if $n=2$, by Lemma 6 , agent 2 survives on all paths in $\Omega^{n}=\Omega^{2}$. 
Assume, thus, that $n>2$. The next lemma considers a given path $\sigma$ and distinguishes two cases: on $\sigma$, either agent 1's consumption exactly coincides with the part of the initial endowment that is nonmeasurable w.r.t. $\Omega^{2}$ or it exceeds it. We show that in both cases, agent $2 \pi$-a.s. survives on $\omega^{2}$ such that $\sigma \in \omega^{2}$.

Q.E.D.

LEMMA 10:onsider a path $\sigma \in \Omega$ and let $\sigma \in \omega^{2}$.

(i) If $\lim _{t \rightarrow \infty} c^{1}\left(\sigma_{t}\right)-e\left(\sigma_{t}\right)+\min _{\tilde{\sigma}_{t} \in \omega_{t}^{2}} e\left(\tilde{\sigma}_{t}\right)=0$, then $\pi$-a.s. 2 survives on $\omega^{2}$.

(ii) If $\lim _{t \rightarrow \infty} \sup c^{1}\left(\sigma_{t}\right)-e\left(\sigma_{t}\right)+\min _{\tilde{\sigma}_{t} \in \omega_{t}^{2}} e\left(\tilde{\sigma}_{t}\right)>0$, then 2 survives on $\omega^{2}$.

PROOF: Part (i). Under condition (i) of the lemma, since $\sum_{j \geq 3} c^{j}\left(\sigma_{t}\right)$ is measurable w.r.t. $\Omega^{3}$, for every $t, c^{2}\left(\sigma_{t}\right)+c^{1}\left(\sigma_{t}\right)-e\left(\sigma_{t}\right)+\min _{\tilde{\sigma}_{t} \in \omega_{t}^{3}} e\left(\tilde{\sigma}_{t}\right) \geq 0$. Hence, $\pi$-a.s.,

$$
\begin{aligned}
\liminf _{t \rightarrow \infty} c^{2}\left(\sigma_{t}\right) & \geq \lim _{t \rightarrow \infty} \inf -c^{1}\left(\sigma_{t}\right)+e\left(\sigma_{t}\right)-\min _{\tilde{\sigma}_{t} \in \omega_{t}^{3}} e\left(\tilde{\sigma}_{t}\right) \\
& =\lim _{t \rightarrow \infty} \sup \left[\min _{\tilde{\sigma}_{t} \in \omega_{t}^{2}} e\left(\tilde{\sigma}_{t}\right)-\min _{\tilde{\sigma}_{t} \in \omega_{t}^{3}} e\left(\tilde{\sigma}_{t}\right)\right]>\epsilon,
\end{aligned}
$$

where the last inequality follows from agent 3's financial constraints being relevant in the limit w.r.t. those of 2 and the fact that each $w^{2}\left(w^{3}\right)$ occurs $\pi$-a.s. i.o. on any $\omega^{2}\left(\omega^{3}\right)$. Hence, agent 2 survives on $\omega^{2} \pi$-a.s.

Part (ii). Since $\sum_{j \geq 2} c^{j}\left(\sigma_{t}\right)$ is measurable w.r.t. $\Omega^{2}, c^{1}\left(\sigma_{t}^{\prime}\right)-e\left(\sigma_{t}^{\prime}\right)+\min _{\tilde{\sigma}_{t} \in \omega_{t}^{2}} e\left(\tilde{\sigma}_{t}\right) \geq$ 0 is also measurable w.r.t. $\Omega^{2}$. Thus, under condition (ii) of the lemma, there is an $\hat{\boldsymbol{\epsilon}}^{1}\left(\omega^{2}\right)$ with $\lim _{t \rightarrow \infty} \sup c^{1}\left(\sigma_{t}^{\prime}\right)-e\left(\sigma_{t}^{\prime}\right)+\min _{\tilde{\sigma}_{t} \in \omega_{t}^{2}} e\left(\tilde{\sigma}_{t}\right)=2 \hat{\boldsymbol{\epsilon}}^{1}\left(\omega^{2}\right)>0$. By measurability, i.o. on $\omega^{2}, \min _{\sigma_{t}^{\prime} \in \omega^{2}} c^{1}\left(\sigma_{t}^{\prime}\right)-e\left(\sigma_{t}^{\prime}\right)+\min _{\tilde{\sigma}_{t} \in \omega_{t}^{2}} e\left(\tilde{\sigma}_{t}\right) \geq \hat{\epsilon}^{1}\left(\omega^{2}\right)$ and, hence, i.o. on $\omega^{2}$, $\sum_{\sigma_{t} \in \omega_{t}^{2}} u_{1}^{\prime}\left(c_{1}\left(\sigma_{t}\right)\right) \pi^{1}\left(\sigma_{t} \mid \omega_{t}^{2}\right) \leq u_{1}^{\prime}\left(\hat{\epsilon}^{1}\left(\omega^{2}\right)\right)<\infty$. Using (11) with $i=2$ and $j=1$, we conclude that agent 2 survives on $\omega^{2}$.

The two parts of Lemma 10 taken together imply that agent $2 \pi$-a.s. survives.

To complete the proof of the proposition we proceed by induction. Take any $h \in$ $\{1 \ldots n-1\}$ such that all agents $1 \ldots h$ a.s. survive. Note that by measurability of agent $h+1$ 's consumption, the total consumption of agents $1 \ldots h$ has to satisfy, for any $\omega^{h+1} \in \Omega^{h+1}$ and any $\sigma \in \omega^{h+1}, \lim _{t \rightarrow \infty} \sum_{i \leq h} c^{i}\left(\sigma_{t}\right)-e\left(\sigma_{t}\right)+\min _{\tilde{\sigma}_{t} \in \omega_{t}^{h+1}} e\left(\tilde{\sigma}_{t}\right) \geq 0$. Q.E.D.

LEMMA 1C:onsider a path $\sigma \in \Omega$ and let $\sigma \in \omega^{h+1}$.

(i) If $\lim _{t \rightarrow \infty} \sum_{i \leq h} c^{i}\left(\sigma_{t}\right)-e\left(\sigma_{t}\right)+\min _{\tilde{\sigma}_{t} \in \omega_{t}^{h+1}} e\left(\tilde{\sigma}_{t}\right)=0$, then $\pi$-a.s. $h+1$ survives on $\omega^{h+1}$.

(ii) If $\lim _{t \rightarrow \infty} \sup \sum_{i \leq h} c^{i}\left(\sigma_{t}\right)-e\left(\sigma_{t}\right)+\min _{\tilde{\sigma}_{t} \in \omega_{t}^{h+1}} e\left(\tilde{\sigma}_{t}\right)>0$, then $h+1$ survives on $\omega^{h+1}$.

PROOF: The proof of part (i) is identical to that of Lemma 10 up to a change in indices and, therefore, is omitted. To prove part (ii), note that by an argument using measurability of consumption and condition (ii) of the lemma analogous to that in the proof of part (ii) of Lemma 10, it can be shown that there is an $\hat{\epsilon}^{h}\left(\omega^{h+1}\right)>0$ such that $\min _{\sigma_{t}^{\prime} \in \omega^{2}} \sum_{i=1}^{h} c^{i}\left(\sigma_{t}^{\prime}\right)-e\left(\sigma_{t}^{\prime}\right)+\min _{\tilde{\sigma}_{t} \in \omega_{t}^{h+1}} e\left(\tilde{\sigma}_{t}\right) \geq \hat{\epsilon}^{h}\left(\omega^{h+1}\right)$ i.o. on $\omega^{h+1}$. Hence, i.o. on $\omega^{h+1}$, at every $\sigma_{t}^{\prime} \in \omega_{t}^{h+1}$, at least one agent $i \in\{1 \ldots h\}$ has to consume at least $\frac{\hat{\epsilon}^{h}\left(\omega^{h+1}\right)}{h}$.

Clearly, if $i$ consumes at least $\hat{\epsilon}^{h}\left(\omega^{h+1}\right)$ on $\sigma_{t}^{\prime}$, measurability implies $c^{i}\left(\sigma_{t}^{\prime}\right)>\frac{\epsilon^{\prime}}{h}$, where $\sigma_{t}^{\prime} \in \omega_{t}^{i} \subseteq \omega_{t}^{h+1}$. Using (11) with $\tilde{j}$ taking the place of $j$ for any $\tilde{j} \in\{1 \ldots i\}$ and, in particu- 
lar, for $j=\tilde{j}=1, \pi^{1}$-a.s., there is a uniform infimum of $u_{1}^{\prime}\left(c^{1}\left(\sigma_{t}^{\prime}\right)\right)$ on $\sigma_{t}^{\prime} \in \omega_{t}^{\prime i}$,

$$
\lim _{t \rightarrow \infty} \inf u_{1}^{\prime}\left(c^{1}\left(\sigma_{t}^{\prime}\right)\right)<\max _{i \in\{1 \ldots h\}} \frac{u_{1}^{\prime}\left(c^{1}\left(\sigma_{0}\right)\right) u_{i}^{\prime}\left(\frac{\hat{\epsilon}^{h}\left(\omega^{h+1}\right)}{h}\right)}{u_{i}^{\prime}\left(c^{i}\left(\sigma_{0}\right)\right)},
$$

and, moreover,

$$
\lim _{t \rightarrow \infty} \inf \max _{\sigma_{t}^{\prime} \in \omega_{t}^{h+1}} u_{1}^{\prime}\left(c^{1}\left(\sigma_{t}^{\prime}\right)\right)<\max _{i \in\{1 \ldots h\}} \frac{u_{1}^{\prime}\left(c^{1}\left(\sigma_{0}\right)\right) u_{i}^{\prime}\left(\frac{\hat{\epsilon}^{h}\left(\omega^{h+1}\right)}{h}\right)}{u_{i}^{\prime}\left(c^{i}\left(\sigma_{0}\right)\right)} .
$$

It follows that

$$
\lim _{t \rightarrow \infty} \inf \sum_{\sigma_{t}^{\prime} \in \omega_{t}^{h+1}} u_{1}^{\prime}\left(c^{1}\left(\sigma_{t}^{\prime}\right)\right) \pi^{1}\left(\sigma_{t}^{\prime} \mid \omega_{t}^{h+1}\right)<\max _{i \in\{1 \ldots h\}} \frac{u_{1}^{\prime}\left(c^{1}\left(\sigma_{0}\right)\right) u_{i}^{\prime}\left(\frac{\hat{\epsilon}^{h}\left(\omega^{h+1}\right)}{h}\right)}{u_{i}^{\prime}\left(c^{i}\left(\sigma_{0}\right)\right)}<\infty .
$$

Using (11) with $i=h+1$ and $j=1$, we have $\lim _{t \rightarrow \infty} \inf u_{h+1}^{\prime}\left(c^{t+1}\left(\omega_{t+1}^{h+1}\right)\right)<\infty$ or $\lim _{t \rightarrow \infty} \sup c^{t+1}\left(\omega_{t+1}^{h+1}\right)>0$, which implies that $h+1$ survives on $\omega^{h+1}$.

We thus conclude that when beliefs and discount factors are identical, all agents a.s. survive.

Q.E.D.

PROPOSITION 17: Consider a population of agents with available sets of assets ordered with respect to inclusion $A^{1} \supset A^{2} \cdots \supset A^{n}$, and, thus, nested partitions, $\Omega^{1}$ strictly finer than $\Omega^{2} \ldots$ strictly finer than $\Omega^{n}$ and ordered survival indices such that

$$
\ln \beta_{i}-\sum_{w^{j}} \pi\left(w^{j}\right) \ln \frac{\pi\left(w^{j}\right)}{\pi^{i}\left(w^{j}\right)}<\ln \beta_{j}-\sum_{w^{j}} \pi\left(w^{j}\right) \ln \frac{\pi\left(w^{j}\right)}{\pi^{j}\left(w^{j}\right)}
$$

holds for all $i<j$. If the constraints of any agent $i \geq 2$ are both relevant in the limit and relevant w.r.t. those of $i-1$, agents 1 and 2 a.s. survive. Furthermore, if for every $j \in\{2 \ldots n-$ $1\}$, such that the survival indices of $j$ and $j+1$ are distinct, all $w^{j+1} \in W^{j+1}$, and all $w^{j} \subseteq w^{j+1}$, $\pi^{j}\left(w^{j} \mid w^{j+1}\right)=\pi\left(w^{j} \mid w^{j+1}\right)$, all agents a.s. survive.

We prove the proposition in a sequence of Lemmata. As in the proof of Proposition 16, w.l.o.g., set $\Omega=\Omega^{1}$.

LemMa 12: Agent 1 survives $\pi$-a.s. and $\pi^{1}$-a.s.

PROOF: The proof that agent 1 survives $\pi$-a.s. is identical to that of Lemma 9. Note that the proof requires that the state $s$ occurs i.o. on almost every path, which is also true for $\pi^{i}$. Hence, agent 1 survives $\pi^{1}$-a.s.

Q.E.D.

LEMMA 13: Agent 2 survives $\pi$-a.s. 
PROOF: Suppose that $\lim _{t \rightarrow \infty} c^{2}\left(\omega_{t}^{2}\right)=0$. Since agent 3's constraints are relevant in the limit w.r.t. those of $2, \pi$-a.s., for $\omega^{3} \supseteq \omega^{2}$, i.o., $\min _{\sigma_{t} \in \omega_{t}^{2}} e\left(\sigma_{t}\right)-\min _{\sigma_{t} \in \omega_{t}^{3}} e\left(\sigma_{t}\right)>\epsilon$. By measurability, $c^{j}\left(\sigma_{t}\right) \leq \min _{\sigma_{t} \in \omega_{t}^{j}} e\left(\sigma_{t}\right)$ for all $j \geq 2, \sigma_{t} \in \omega_{t}^{j}, \omega_{t}^{j} \supseteq \omega^{2}$. Hence, i.o. on $\omega^{2}$, $c^{1}\left(\sigma_{t}\right) \geq \epsilon$ for all $\sigma_{t} \in \omega_{t}^{2}$. However, since agent 1's survival index is strictly lower than that of $2, \pi$-a.s., this contradicts (10) (with $i=2, j=1$ ), since the infimum of the 1.h.s. would be nonpositive, whereas the r.h.s. is strictly positive. Hence, agent $2 \pi$-a.s. survives. Q.E.D.

The following lemma uses the condition that for agents $2 \ldots n-1$, conditional beliefs on the next coarser partition are correct.

LEMMA 14: Suppose that for every $j \in\{2 \ldots n-1\}$, all $w^{j+1} \in W^{j+1}$, and all $w^{j} \subseteq w^{j+1}$, $\pi^{j}\left(w^{j} \mid w^{j+1}\right)=\pi\left(w^{j} \mid w^{j+1}\right)$. For any $j \in\{1 \ldots n-1\}, \lim _{t \rightarrow \infty}\left|c^{j}\left(\sigma_{t}\right)-\bar{c}^{j}\left(\sigma_{t}\right)\right|=0 \pi$-a.s. on $\Omega$, where for $\sigma_{t} \in \omega_{t}^{j} \subseteq \omega_{t}^{j+1}, \bar{c}^{j}\left(\sigma_{t}\right)=\min _{\tilde{\sigma}_{t} \in \omega_{t}^{j}} e\left(\tilde{\sigma}_{t}\right)-\min _{\tilde{\sigma}_{t} \in \omega_{t}^{j+1}} e\left(\tilde{\sigma}_{t}\right)$. Hence, j survives a.s. if $j+1$ 's financial constraints are relevant in the limit w.r.t. those of $j$ and vanishes a.s. if they are irrelevant in the limit w.r.t. those of $j$.

Proof: Consider first agent 1. Using condition (10) with $i=2$ and $j=1$, and noting that the r.h.s. is strictly positive, we obtain that $\pi$-a.s.,

$$
\lim _{T \rightarrow \infty} \sum_{\tilde{\sigma}_{T+1} \in \omega_{T+1}^{2}} u_{1}^{\prime}\left(c^{1}\left(\tilde{\sigma}_{T+1}\right)\right) \pi^{1}\left(\tilde{\sigma}_{T+1} \mid \omega_{T+1}^{2}\right)=\infty
$$

By measurability, for every $\omega_{t}^{2} \in \Omega^{2}, c_{t}^{1}\left(\sigma_{t}\right)=c^{1}\left(\sigma_{t}^{\prime}\right)$ for all $\sigma_{t}, \sigma_{t}^{\prime} \in \hat{\Omega}_{t}^{1}\left(\omega_{t}^{2}\right)$. As shown in Lemma $12, \pi$-a.s. and $\pi^{1}$-a.s., on every $\sigma$, denoting by $\bar{c}_{t}^{1}\left(\sigma_{t}\right)=e\left(\sigma_{t}\right)-\min _{\sigma_{t} \in \omega_{t}^{2}} e\left(\sigma_{t}\right)$,

$$
\lim _{t \rightarrow \infty} \inf c_{t}^{1}\left(\sigma_{t}\right)-\bar{c}_{t}^{1}\left(\sigma_{t}\right) \geq 0 .
$$

Hence, $\pi$-a.s. for every $\omega^{2}$,

$$
\begin{aligned}
\lim _{T \rightarrow \infty} & \sum_{\tilde{\sigma}_{T+1} \in \omega_{T+1}^{2}} u_{1}^{\prime}\left(c^{1}\left(\tilde{\sigma}_{T+1}\right)\right) \pi^{1}\left(\tilde{\sigma}_{T+1} \mid \omega_{T+1}^{2}\right) \\
= & \lim _{T \rightarrow \infty}\left[\sum_{\tilde{\sigma}_{T+1} \in \hat{\Omega}_{T+1}^{1}\left(\omega_{T+1}^{2}\right)} u_{1}^{\prime}\left(c^{1}\left(\tilde{\sigma}_{T+1}\right)\right) \pi^{1}\left(\tilde{\sigma}_{T+1} \mid \omega_{T+1}^{2}\right)\right. \\
& \left.+\sum_{\tilde{\sigma}_{T+1} \in \tilde{\Omega}_{T+1}^{1}\left(\omega_{T+1}^{2}\right)} u_{1}^{\prime}\left(c^{1}\left(\tilde{\sigma}_{T+1}\right)\right) \pi^{1}\left(\tilde{\sigma}_{T+1} \mid \omega_{T+1}^{2}\right)\right] \\
= & \lim _{T \rightarrow \infty}\left[u_{1}^{\prime}\left(c^{1}\left(\omega_{T+1}^{2}\right)\right) \pi^{1}\left(\hat{\Omega}_{T+1}^{1}\left(\omega_{T+1}^{2}\right)\right)+\sum_{\tilde{\sigma}_{T+1} \in \tilde{\Omega}_{T+1}^{1}\left(\omega_{T+1}^{2}\right)} u_{1}^{\prime}\left(c^{1}\left(\tilde{\sigma}_{T+1}\right)\right) \pi^{1}\left(\tilde{\sigma}_{T+1} \mid \omega_{T+1}^{2}\right)\right],
\end{aligned}
$$

where the first term uses $c^{1}\left(\tilde{\sigma}_{T+1}\right)=c^{1}\left(\tilde{\sigma}_{T+1}^{\prime}\right)$ for all $\tilde{\sigma}_{T+1}, \tilde{\sigma}_{T+1}^{\prime} \in \hat{\Omega}_{T+1}^{1}\left(\omega_{T+1}^{2}\right)$. Note further that since agent 2's financial constraints are relevant w.r.t. those of 1 in the limit, and 
since every $w^{2}$ occurs i.o. $\pi$-a.s. on every $\omega^{2}$,

$$
\begin{aligned}
& \lim _{T \rightarrow \infty} \inf \sum_{\tilde{\sigma}_{T+1} \in \tilde{\Omega}_{T+1}^{1}\left(\omega_{T+1}^{2}\right)} u_{1}^{\prime}\left(c^{1}\left(\tilde{\sigma}_{T+1}\right)\right) \pi^{1}\left(\tilde{\sigma}_{T+1} \mid \omega_{T+1}^{2}\right) \\
& \quad \leq \lim _{T \rightarrow \infty} \inf \sum_{\tilde{\sigma}_{T+1} \in \tilde{\Omega}_{T+1}^{1}\left(\omega_{T+1}^{2}\right)} u_{1}^{\prime}\left(\bar{c}^{1}\left(\tilde{\boldsymbol{\sigma}}_{T+1}\right)\right) \pi^{1}\left(\tilde{\boldsymbol{\sigma}}_{T+1} \mid \omega_{T+1}^{2}\right) \leq u_{1}^{\prime}(\boldsymbol{\epsilon}) .
\end{aligned}
$$

Since $\pi$-a.s., $\lim _{T \rightarrow \infty} \sum_{\tilde{\boldsymbol{\sigma}}_{T+1} \in \omega_{T+1}^{2}} u_{1}^{\prime}\left(c^{1}\left(\tilde{\boldsymbol{\sigma}}_{T+1}\right)\right) \pi^{1}\left(\tilde{\boldsymbol{\sigma}}_{T+1} \mid \omega_{T+1}^{2}\right)=\infty$, we thus have that $\lim _{T \rightarrow \infty} u_{1}^{\prime}\left(c^{1}\left(\tilde{\omega}_{T+1}^{2}\right)\right) \pi^{1}\left(\hat{\Omega}_{T+1}^{1}\left(\omega_{T+1}^{2}\right)\right)=\infty$ and, hence, $c^{1}\left(\left(\tilde{\omega}_{T+1}^{2}\right)\right) \rightarrow 0 \pi$-a.s. on $\hat{\Omega}_{T+1}^{1}\left(\omega_{T+1}^{2}\right)$.

By (15) and since $e\left(\sigma_{t}\right)-c_{1}\left(\sigma_{t}\right)$ is $\Omega^{2}$-measurable, $\lim _{T \rightarrow \infty}\left|c^{1}\left(\sigma_{T+1}\right)-\bar{c}^{1}\left(\sigma_{T+1}\right)\right|=0$, $\pi$-a.s.

We can now repeat the argument by induction for any agent with an index greater than 1. For agent $j>1$, define $\bar{c}_{t}^{j}\left(\omega_{t}^{j}\right)=\min _{\sigma_{t} \in \omega_{t}^{j}} e\left(\sigma_{t}\right)-\min _{\sigma_{t} \in \omega_{t}^{j+1}} e\left(\sigma_{t}\right)$. Suppose that $\pi$-a.s., $\lim _{t \rightarrow \infty} c^{j}\left(\sigma_{t}\right)=\lim _{t \rightarrow \infty}^{j} \bar{c}^{i}\left(\sigma_{t}\right)$ for all $j \leq k$. Then, $\pi$-a.s.,

$$
\lim _{t \rightarrow \infty} \inf c_{t}^{k+1}\left(\omega_{t}^{k+1}\right)-\bar{c}_{t}^{k+1}\left(\omega_{t}^{k+1}\right) \geq 0,
$$

where for any $\bar{\sigma}_{t} \in \omega_{t}^{k+1} \subseteq \omega_{t}^{k+2}$,

$$
\bar{c}_{t}^{k+1}\left(\omega_{t}^{k+1}\right)=\min _{\sigma_{t} \in \omega_{t}^{k+1}} e\left(\sigma_{t}\right)-\min _{\sigma_{t} \in \omega_{t}^{k+2}} e\left(\sigma_{t}\right)=e\left(\bar{\sigma}_{t}\right)-\sum_{j=1}^{k} \bar{c}_{j}\left(\bar{\sigma}_{t}\right)-\min _{\sigma_{t} \in \omega_{t}^{k+2}} e\left(\sigma_{t}\right)
$$

and, furthermore, since $k+2$ 's financial constraint is relevant in the limit w.r.t. that of $k+1$, on almost all $\omega_{T+1}^{k+2}$,

$$
\lim _{t \rightarrow \infty} \sup \bar{c}_{k+1}>\epsilon .
$$

Furthermore, for any $\omega_{t}^{k+1} \in \hat{\Omega}_{t}^{k+1}\left(\omega_{t}^{k+2}\right), e\left(\sigma_{t}\right)-\sum_{j \leq k} \bar{c}_{t}^{j}\left(\sigma_{t}\right)=e\left(\sigma_{t}^{\prime}\right)-\sum_{j \leq k} \bar{c}_{t}^{j}\left(\sigma_{t}^{\prime}\right)$ for all $\sigma_{t}, \sigma_{t}^{\prime} \in \omega_{t}^{k+1}$. Finally, for any $\omega_{t}^{k+2}$, any $j>k+1$, any $\omega_{t}^{k+1}, \omega_{t}^{\prime k+1} \in \hat{\Omega}_{t}^{k+1}\left(\omega_{t}^{k+2}\right)$, and for any $\sigma_{t} \in \omega_{t}^{k+1}, \sigma_{t}^{\prime} \in \omega_{t}^{\prime k+1}, c^{j}\left(\sigma_{t}\right)=c^{j}\left(\sigma_{t}^{\prime}\right)$. It thus follows that for every $\epsilon>0$,

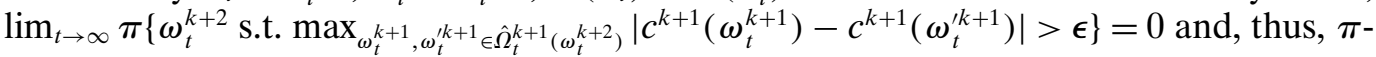
a.s. for every $\omega^{k+2}$, in the limit, $k+1$ 's consumption is constant on $\hat{\Omega}_{t}^{k+1}\left(\omega_{t}^{k+2}\right)$ :

$$
\lim _{t \rightarrow \infty} \pi\left(\max _{\omega_{t}^{k+1}, \omega_{t}^{\prime k+1} \in \hat{\Omega}_{t}^{k+1}\left(\omega_{t}^{k+2}\right)}\left|c^{k+1}\left(\omega_{t}^{k+1}\right)-c^{k+1}\left(\omega_{t}^{\prime k+1}\right)\right|=0 \mid \omega_{t}^{k+2}\right)=1 .
$$

Since $k+1$ 's survival index is strictly smaller than that of $k+2$, using (10) with $i=k+2$ and $j=k+1$, and noting that the r.h.s. is strictly positive, we obtain $\pi$-a.s., $\lim _{T \rightarrow \infty} \sum_{\tilde{\omega}_{T+1}^{k+1} \subseteq \omega_{T+1}^{k+2}} u_{k+1}^{\prime}\left(c^{k+1}\left(\tilde{\omega}_{T+1}^{k+1}\right)\right) \pi^{k+1}\left(\tilde{\omega}_{T+1}^{k+1} \mid \omega_{T+1}^{k+2}\right)=\infty$.

Then, since $\pi^{k+1}\left(\tilde{\omega}_{T+1}^{k+1} \mid \omega_{T+1}^{k+2}\right)=\pi\left(\tilde{\omega}_{T+1}^{k+1} \mid \omega_{T+1}^{k+2}\right)$, and using (18),

$$
\lim _{T \rightarrow \infty} \sum_{\tilde{\omega}_{T+1}^{k+1} \subseteq \omega_{T+1}^{k+2}} u_{k+1}^{\prime}\left(c^{k+1}\left(\tilde{\omega}_{T+1}^{k+1}\right)\right) \pi^{k+1}\left(\tilde{\omega}_{T+1}^{k+1} \mid \omega_{T+1}^{k+2}\right)
$$




$$
\begin{aligned}
= & \lim _{T \rightarrow \infty}\left[\sum_{\tilde{\omega}_{T+1}^{k+1} \in \hat{\Omega}_{T+1}^{k+1}\left(\omega_{T+1}^{k+2}\right)} u_{k+1}^{\prime}\left(c^{k+1}\left(\tilde{\omega}_{T+1}^{k+1}\right)\right) \pi\left(\tilde{\omega}_{T+1}^{k+1} \mid \omega_{T+1}^{k+2}\right)\right. \\
& \left.+\sum_{\tilde{\omega}_{T+1}^{k+1} \tilde{\Omega}_{T+1}^{k+1}\left(\omega_{T+1}^{k+2}\right)} u_{k+1}^{\prime}\left(c^{k+1}\left(\tilde{\omega}_{T+1}^{k+1}\right)\right) \pi\left(\tilde{\omega}_{T+1}^{k+1} \mid \omega_{T+1}^{k+2}\right)\right] \\
= & \lim _{T \rightarrow \infty}\left[u_{k+1}^{\prime}\left(c^{k+1}\left(\tilde{\omega}_{T+1}^{k+2}\right)\right) \pi\left(\hat{\Omega}_{T+1}^{k+1}\left(\omega_{T+1}^{k+2}\right)\right)\right. \\
& \left.+\sum_{\tilde{\omega}_{T+1}^{k+1} \tilde{\Omega}_{T+1}^{k+1}\left(\omega_{T+1}^{k+2}\right)} u_{k+1}^{\prime}\left(c^{k+1}\left(\tilde{\omega}_{T+1}^{k+1}\right)\right) \pi\left(\tilde{\omega}_{T+1}^{k+1} \mid \omega_{T+1}^{k+2}\right)\right] .
\end{aligned}
$$

Furthermore, (16) together with (17), the fact that $k+2$ 's constraints are relevant in the limit w.r.t. $k+1$ 's, and the fact that each $w^{k+2}$ occurs i.o. $\pi$-a.s., implies that $\pi$-a.s.,

$$
\begin{aligned}
& \lim _{T \rightarrow \infty} \inf \sum_{\tilde{\omega}_{T+1}^{k+1} \in \tilde{\Omega}_{T+1}^{k+1}\left(\omega_{T+1}^{k+2}\right)} u_{k+1}^{\prime}\left(c^{k+1}\left(\tilde{\omega}_{T+1}^{k+1}\right)\right) \pi\left(\tilde{\omega}_{T+1}^{k+1} \mid \omega_{T+1}^{k+2}\right) \\
& \quad \leq \lim _{T \rightarrow \infty} \inf \sum_{\tilde{\omega}_{T+1}^{k+1} \in \tilde{\Omega}_{T+1}^{k+1}\left(\omega_{T+1}^{k+2}\right)} u_{k+1}^{\prime}\left(\bar{c}^{k+1}\left(\tilde{\omega}_{T+1}^{k+1}\right)\right) \pi\left(\tilde{\omega}_{T+1}^{k+1} \mid \omega_{T+1}^{k+2}\right) \leq u_{\tilde{k}-1}^{\prime}(\varepsilon) .
\end{aligned}
$$

Since $\pi$-a.s., $\lim _{T \rightarrow \infty} \sum_{\tilde{\omega}_{T+1}^{k+1} \subseteq \omega_{T+1}^{k+2}} u_{k+1}^{\prime}\left(\bar{c}^{k+1}\left(\tilde{\omega}_{T+1}^{k+1}\right)\right) \pi\left(\tilde{\omega}_{T+1}^{k+1} \mid \omega_{T+1}^{k+2}\right)=\infty$, we, thus, have that $\pi$-a.s., $\lim _{T \rightarrow \infty} u_{k+1}^{\prime}\left(c^{k+1}\left(\tilde{\omega}_{T+1}^{k+2}\right)\right) \pi\left(\hat{\Omega}_{T+1}^{k+1}\left(\omega_{T+1}^{k+2}\right)\right)=\infty$ and, hence, $\pi$-a.s. on $\hat{\Omega}_{T+1}^{k+1}\left(\omega_{T+1}^{k+2}\right), c^{k+1}\left(\tilde{\omega}_{T+1}^{k+2}\right) \rightarrow 0$.

Since $e\left(\sigma_{t}\right)-\sum_{j \leq k+1} \bar{c}^{j}\left(\sigma_{t}\right)$ is measurable w.r.t. $\Omega^{k+2}, \sum_{j \geq k+2} c^{j}\left(\sigma_{t}\right)$ is measurable w.r.t. $\Omega^{k+2}, c^{k+1}\left(\tilde{\omega}_{T+1}^{k+2}\right) \rightarrow 0, \pi$-a.s., and $\lim _{T \rightarrow \infty}\left|c^{j}\left(\sigma_{T}\right)-\bar{c}^{j}\left(\sigma_{T}\right)\right|=0, \pi$-a.s., $j \leq k$, we have $\pi$ a.s., $\lim _{T \rightarrow \infty}\left|c^{k+1}\left(\sigma_{T}\right)-\bar{c}^{k+1}\left(\sigma_{T}\right)\right|=0$. It follows that $k+1$ a.s. survives if his constraints are relevant in the limit w.r.t. those of $k+2$. In contrast, if $k+1$ 's constraints are irrelevant in the limit w.r.t. those of $k+2$, we have that a.s., $\lim _{T \rightarrow \infty} \check{\Omega}_{T+1}^{k+1}\left(\omega_{T+1}^{k+2}\right)=\emptyset$ and, hence, $\pi$ a.s., $c^{k+1}\left(\omega_{T+1}^{k+1}\right) \rightarrow 0$ so that agent $k+1$ a.s. vanishes.

Q.E.D.

LEMMA 15: Agent $n$ a.s. survives.

PROOF: We have shown above that for agents $j \in\{1 \ldots n-1\}$, consumption converges a.s. to $\bar{c}^{j}$. It follows that for $\sigma_{t} \in \omega_{t}^{n}, \pi$-a.s.,

$$
\lim _{t \rightarrow \infty} c^{n}\left(\sigma_{t}\right)=e\left(\sigma_{t}\right)-\sum_{j=1}^{n-1} \bar{c}^{j}\left(\sigma_{t}\right)=\min _{\tilde{t}_{t} \in \omega_{t}^{n}} e\left(\tilde{\sigma}_{t}\right) \geq m
$$

and, hence, $n$ a.s. survives.

Q.E.D.

\section{Proof of Proposition 6}

LEMMA 16: Suppose that agents $j \ldots j+k$ have equal discount factors and identical beliefs, whereas $j+k+1$ has a strictly higher survival index. Suppose that either $j=1$ or $j>1$, $j-1$ has a strictly lower survival index than $j$, and a.s., $\lim _{t \rightarrow \infty} \sum_{i<j}\left[c^{i}\left(\sigma_{t}\right)-\bar{c}^{i}\left(\sigma_{t}\right)\right]=0$. Then $j \ldots j+k$ a.s. survive and a.s. $\lim _{t \rightarrow \infty} \sum_{i=j}^{j+k}\left[c^{i}\left(\sigma_{t}\right)-\bar{c}^{i}\left(\sigma_{t}\right)\right]=0$. 
PROOF: Suppose in a manner of contradiction that for some $\sigma$,

$$
\lim _{t \rightarrow \infty} \sup \sum_{i=j}^{j+k}\left[c^{i}\left(\sigma_{t}\right)-\bar{c}^{i}\left(\sigma_{t}\right)\right]>0
$$

The same arguments as those used in the proof of part (ii) of Lemma 11 can be used to establish that for $\omega^{j+k+1} \in \Omega^{j+k+1}$ such that $\sigma \in \omega^{j+k+1}$, there is a $\hat{\epsilon}\left(\omega^{j+k+1}\right)>0$ such that

$$
\lim _{t \rightarrow \infty} \inf \sum_{\omega_{t}^{j} \subseteq \omega_{t}^{j+k+1}} u_{j}^{\prime}\left(c^{j}\left(\omega_{t}^{j}\right)\right) \pi^{j}\left(\omega_{t}^{j} \mid \omega_{t}^{j+k+1}\right)<\max _{i \in\{j \ldots j+k\}} \frac{u_{j}^{\prime}\left(c^{j}\left(\sigma_{0}\right)\right) u_{i}^{\prime}\left(\frac{\hat{\boldsymbol{\epsilon}}\left(\omega^{j+k+1}\right)}{h}\right)}{u_{i}^{\prime}\left(c^{i}\left(\sigma_{0}\right)\right)} .
$$

However, using condition (10) with $j=j$ and $i=j+k+1$, and noting that the r.h.s. is strictly positive, implies $\pi$-a.s., $\lim _{t \rightarrow \infty} \sum_{\tilde{\omega}_{t}^{j} \subseteq \omega_{t}^{j+k+1}} u_{j}^{\prime}\left(c^{j}\left(\tilde{\omega}_{t}^{j}\right)\right) \pi^{j}\left(\tilde{\omega}_{t}^{j} \mid \omega_{t}^{j+k+1}\right)=\infty$, thus establishing a contradiction to $\lim _{t \rightarrow \infty} \sup \sum_{i=j}^{j+k}\left[c^{i}\left(\sigma_{t}\right)-\bar{c}^{i}\left(\sigma_{t}\right)\right]>0$.

It follows that $\lim _{t \rightarrow \infty} \sum_{i=j}^{j+k}\left[c^{i}\left(\sigma_{t}\right)-\bar{c}^{i}\left(\sigma_{t}\right)\right]=0$. Since $j+1$ 's constraints are relevant in the limit w.r.t. those of $j$, agent $j$ a.s. survives. We can then apply Lemmata 10 and 11 to show that all agents $j+1 \ldots j+k$ survive a.s. as well.

The proof of Proposition 6 now follows by combining Propositions 16 and 17 with the result of Lemma 16. Without loss of generality, let agents $1 \ldots h_{1}$ for $h_{1} \in\{1 \ldots n\}$ have distinct survival indices. In particular, agent 1 a.s. survives. By Lemma 14, we know that if agents $1 \ldots h_{1}, h_{1} \in\{1 \ldots n\}$ have distinct ordered survival indices, they all survive a.s. and, furthermore, $\lim _{t \rightarrow \infty}\left[c^{i}\left(\sigma_{t}\right)-\bar{c}^{i}\left(\sigma_{t}\right)\right]=0$ a.s. obtains for all $i \in\left\{1 \ldots h_{1}\right\}$. If $h_{1}=n$, then Proposition 17 completes the proof. If $h_{1}<n$, let agent $h_{2}-1$ be the agent with the largest index who has a discount factor and beliefs identical to those of agent $h_{1}$. Then by Proposition 16 and Lemma 16, all agents $h_{1} \ldots h_{2}-1$ a.s. survive and, furthermore, the condition $\lim _{t \rightarrow \infty} \sum_{i=1}^{h_{2}-1}\left[c^{i}\left(\sigma_{t}\right)-\bar{c}^{i}\left(\sigma_{t}\right)\right]=0$ a.s. obtains. If $h_{2}-1=n$, this completes the proof.

If $h_{2}-1<n$, proceed by induction. Suppose that agents $1 \ldots h_{k}-1$ a.s. survive and a.s. $\sum_{i=1}^{h_{k}-1}\left[c^{i}\left(\sigma_{t}\right)-\bar{c}^{i}\left(\sigma_{t}\right)\right]=0$. If $h_{k}=n, h_{k}$ a.s. survives by Lemma 15. If $h_{k}<n$ and $h_{k}$ has a survival index distinct from $h_{k}+1$, then the proof of Lemma 14 (applied to agents $h_{k}$ and $\left.h_{k+1}\right)$ shows that agent $h_{k}$ a.s. survives and a.s. $\lim _{t \rightarrow \infty}\left[c^{h_{k}}\left(\sigma_{t}\right)-\bar{c}^{h_{k}}\left(\sigma_{t}\right)\right]=$ 0 . If, in contrast, agents $h_{k} \ldots h_{k+1}-1$ have identical discount factors and beliefs, then by Lemma 16 and Proposition 16 and, all agents $h_{k} \ldots h_{k+1}-1$ a.s. survive and, furthermore, the condition $\lim _{t \rightarrow \infty} \sum_{i=1}^{h_{k+1}-1}\left[c^{i}\left(\sigma_{t}\right)-\bar{c}^{i}\left(\sigma_{t}\right)\right]=0$ a.s. obtains. This proves Proposition 6 . Q.E.D.

\section{Proof of Proposition 7}

Note that for all agents other than $j, c^{i}\left(\sigma_{t} ; s\right)=c^{i}\left(\sigma_{t} ; s^{\prime}\right)$ has to hold in equilibrium. Since condition (4) is satisfied, agent $j$ 's consumption on state $s$ is bounded below by $\epsilon$ in the limit. Since state $s$ occurs i.o. $\pi$-.a.s., we conclude that $j$ survives a.s.

Whenever the economy has nonnested partitions as in Definition 5, the same argument applies to every agent $j \in I$ and the statement of the proposition obtains. 


\section{Proof of Proposition 8}

Consider a set of paths $\bar{\Omega}$ that has a strictly positive probability and on which the unconstrained agent $j$ vanishes a.s. We know from the previous result that, on this set of paths, all constrained agents survive a.s. Hence, take the minimal set $\bar{\Omega}^{\prime}$ such that $\bar{\Omega}^{\prime} \supseteq \bar{\Omega}$ and $\bar{\Omega}^{\prime}$ is measurable w.r.t. $\Omega^{i}$ for some constrained agent $i$. The set $\bar{\Omega}^{\prime}$ also has a strictly positive probability and since $i$ survives a.s. on $\bar{\Omega}$, he also survives a.s. on $\bar{\Omega}^{\prime}$. It follows that for any path $\omega^{i} \subseteq \bar{\Omega}^{\prime}$, the numerator of (11) has a finite infimum and, hence, the denominator has to have a finite infimum. Hence, as shown in the proof of Proposition 3, the unconstrained agent $j$ survives a.s. on every such $\omega^{i} \subseteq \bar{\Omega}^{\prime}$, a contradiction. We conclude, thus, that agent $j$ survives a.s.

Since $j$ survives a.s., we know from the argument used in the proof of Proposition 3 that any agent who is more constrained than $j$, has correct beliefs, and has an identical discount factor also survives a.s.

\section{Proof of Proposition 9}

The existence of such an equilibrium follows easily from Bewley's (1972) theorem. If all agents can trade on $\sigma_{t^{*}}^{*}$, then all agents assign 0 probability to all $\sigma_{t} \notin \Omega_{\sigma_{t^{*}}^{*}}$ and we can directly apply the result of Proposition 1 . If not all agents can trade on $\sigma_{t^{*}}^{*}$, then the set of contingencies in this economy includes all $\sigma_{t} \in \omega_{t}^{* i}$ for all $\omega_{t}^{* i} \in \Omega_{\sigma_{t^{*}}^{* i}}^{* i}$ for some $i \in I$. Obviously, given $\sigma_{t^{*}}^{*}$, many of the paths have an objective probability of 0 and are assigned 0 probability by agents who can trade on $\sigma_{t^{*}}^{*}$. In contrast, agents who cannot trade on $\sigma_{t^{*}}^{*}$ assign (mistakenly) strictly positive probability to impossible events. Hence, in equilibrium, there will potentially be trade over 0 probability contingencies: agents who can trade on $\sigma_{t^{*}}^{*}$ will want to sell consumption contingent on $\sigma_{t} \notin \Omega_{\sigma_{t^{*}}^{*}}$, whereas agents who cannot trade on $\sigma_{t^{*}}^{*}$ would like to buy it. Nonnegativity constraints on consumption ensure that such trades remain finite. Given Assumption 3, all agents will wish to assign strictly positive consumption to nodes $\sigma_{t}^{*} \in \Omega_{\sigma_{t^{*}}^{*}}$ as long as $p\left(\sigma_{t}^{*}\right) \in(0 ; \infty)$. However, if $p\left(\sigma_{t}\right) \in(0 ; \infty)$, only an agent $j$ for whom $\sigma_{t} \in \omega_{t}^{* j} \backslash \Omega_{\sigma_{t^{*}}^{*}}$ will wish to assign strictly positive consumption to such a node, whereas all agents who can trade on $\sigma_{t^{*}}^{*}$ will want to consume 0 at $\sigma_{t}$. We will now show that this cannot constitute an equilibrium allocation and, hence, $p\left(\sigma_{t}\right)=0$ has to hold, whenever $\sigma_{t}^{*} \notin \Omega_{\sigma_{t^{*}}^{*}}$.

Take $\sigma_{t} \notin \Omega_{\sigma_{t^{*}}^{*}}$ such that there is an $l \in I$ and an $\omega_{t}^{* l} \in \Omega_{\sigma_{t^{*}}^{*}}^{* l}$ such that $\sigma_{t} \in \omega_{t}^{* l}$. Let $L$ denote the set of all such $l$. Suppose that $p\left(\sigma_{t}\right)>0$ and, thus, $c^{* k}\left(\sigma_{t}\right)=0$ for all $k \notin L$. Then $\sum_{l \in L} c^{* l}\left(\sigma_{t}\right)=e\left(\sigma_{t}\right)$. Let $l^{\prime}$ be the agent with the finest partition in $L$. Then there is also a node $\sigma_{t}^{*} \in \Omega_{\sigma_{t^{*}}^{*}}$ such that $\sigma_{t}$ and $\sigma_{t}^{*} \in \omega_{t}^{* l^{\prime}}$, and, hence, $\sigma_{t}^{*} \in \omega_{t}^{* l}$ for all $l \in L$. By Assumption 4, we can choose $\sigma_{t}^{*}$ so that $e\left(\sigma_{t}^{*}\right) \leq e\left(\sigma_{t}\right)$. Measurability of consumption implies that $\sum_{l \in L} c^{* l}\left(\sigma_{t}\right)=\sum_{l \in L} c^{* l}\left(\sigma_{t}^{*}\right)$. However, $c^{* k}\left(\sigma_{t}\right)=0<c^{* k}\left(\sigma_{t}^{*}\right)$ for all $k$ who can trade on $\sigma_{t^{*}}^{*}$; hence, this cannot be an equilibrium allocation. We conclude that $p\left(\sigma_{t}\right)=0$ for all $\sigma_{t} \notin \Omega_{\sigma_{t^{*}}^{*}}$.

\section{Proof of Lemma 1}

To show that prices are uniformly bounded away from 0 , we use an argument similar to Krebs (2004). On an optimal consumption path for any consumer $i$, we have to have

$$
q_{\min }\left(\sigma_{t} ; w\right) u_{i}^{\prime}\left(c^{i}\left(\sigma_{t}\right)\right) \geq \beta_{i} \sum_{s \in w} u_{i}^{\prime}\left(c^{i}\left(\sigma_{t} ; s\right)\right) \pi^{i}(s) .
$$


Note that (i) in an economy with $n$ agents, there is at least one agent $i$ who consumes at least $\frac{1}{n}$ of the minimal initial endowment of the economy, $c^{i}\left(\sigma_{t}\right) \geq \tilde{m}$; (ii) for any agent and at any node, $c^{i}\left(\sigma_{t} ; s\right) \leq m^{\prime}$, which is the maximal total endowment of the economy; (iii) $\pi^{i}(s) \geq \underline{\pi}>0$ for all $i$ and all $s \in S$. It thus follows that the uniform lower bound on equilibrium asset prices is given by

$$
q_{\min }\left(\sigma_{t} ; w\right) \geq \min _{w \in W^{0}} \min _{i \in I} \beta_{i} \sum_{s \in w} \frac{u_{i}^{\prime}\left(m^{\prime}\right)}{u_{i}^{\prime}(\tilde{m})} \underline{\pi}>0 .
$$

It follows that the price of asset $w$ is uniformly bounded below by

$$
\underline{q}(w)=\min _{i \in I} \beta_{i} \sum_{s \in w} \frac{u_{i}^{\prime}\left(m^{\prime}\right)}{u_{i}^{\prime}(\tilde{m})} \underline{\pi}
$$

and the price of the bond is uniformly bounded below by

$$
\underline{q}=\min _{i \in I} \beta_{i} \sum_{s \in S} \frac{u_{i}^{\prime}\left(m^{\prime}\right)}{u_{i}^{\prime}(\tilde{m})} \underline{\pi} .
$$

The uniform upper bound can be determined by using the Magill and Quinzii (1994, pp. 859-860) condition A4, which is always satisfied for agents with expected utility preferences. Let $\tilde{\beta}$ denote the uniform coefficient of patience introduced in A4 of Magill and Quinzii (1994). They show that $\sum_{w \in W^{0}} q\left(\sigma_{t} ; w\right) \leq \bar{q}=\frac{1}{(1-\tilde{\beta}) \tilde{m}}$ is a uniform upper bound on the equilibrium price of the bond in the economy. It then follows that $q\left(\sigma_{t} ; w\right) \leq \frac{1}{(1-\tilde{\beta}) \tilde{m}}$ for any $\sigma_{t}$ and any $w$.

\section{Proof of Lemma 2}

Suppose that for every $N>0$, there is an $i,\left(\sigma_{t} ; \tilde{w}\right)$ such that $\left|\theta_{i}\left(\sigma_{t} ; \tilde{w}\right)\right|>N$. Suppose, for example, $\theta_{i}\left(\sigma_{t} ; \tilde{w}\right)>N$. Since $\sum_{w \in W^{i}} q\left(\sigma_{t} ; w\right) \theta_{i}\left(\sigma_{t} ; w\right) \leq D$ and since, by the proof of Lemma $1, q\left(\sigma_{t} ; w\right) \in[\underline{q} ; \bar{q}]$ with $\underline{q}>0$, it follows that

$$
\begin{aligned}
\underline{q} N+\sum_{w \in W^{i} \backslash \tilde{w}} q\left(\sigma_{t} ; w\right) \theta_{i}\left(\sigma_{t} ; w\right) & \leq D, \\
\sum_{w \in W^{i} \backslash \tilde{w}} q\left(\sigma_{t} ; w\right) \theta_{i}\left(\sigma_{t} ; w\right) & \leq D-\underline{q} N,
\end{aligned}
$$

and since $N$ can be chosen large enough so that $D-q N<0$, there must be at least one $\bar{w}$ such that $\theta_{i}\left(\sigma_{t} ; \bar{w}\right) \leq \frac{D-q N}{\bar{q}}$. It then follows that in state $\left(\sigma_{t} ; s\right)$ with $s \in \bar{w}$, the value of debt of the agent has to be at least

$$
\theta_{i}\left(\left(\sigma_{t} ; s\right) ; w\right) q\left(\left(\sigma_{t} ; s\right) ; w\right) \geq \frac{\underline{q} N-D}{\bar{q}}-e^{i}\left(\sigma_{t} ; s\right) .
$$

Hence, for $N>\frac{m^{\prime} \bar{q}+D(\bar{q}+1)}{q}$, we obtain a contradiction to the uniform bound on the value of debt.

The argument for $\theta_{i}\left(\sigma_{t} ; \tilde{w}\right)<-N$ is symmetric. 


\section{Proof of Proposition 11}

From the definition of $\hat{r}_{i}^{j}$, we have

$$
\begin{aligned}
\prod_{t=1}^{T+1} \hat{r}_{i}^{j}\left(\sigma_{t}\right) & =\prod_{t=1}^{T+1} \frac{u_{i}^{\prime}\left(c^{i}\left(\sigma_{t}\right)\right)}{\sum_{s \in w_{t}^{j}} u_{i}^{\prime}\left(c^{i}\left(\sigma_{t-1} ; s\right)\right) \pi^{i}\left(s \mid w_{t}^{j}\right)} \\
& =\beta_{i}^{T+1} \frac{u_{i}^{\prime}\left(c^{i}\left(\sigma_{T+1}\right)\right)}{u_{i}^{\prime}\left(c^{i}\left(\sigma_{0}\right)\right)} \prod_{t=0}^{T} \pi^{i}\left(w^{j}\left(\sigma_{t+1}\right)\right) \frac{u_{i}^{\prime}\left(c^{i}\left(\sigma_{t}\right)\right)}{\beta_{i} \sum_{s \in w^{j}\left(\sigma_{t+1}\right)} u_{i}^{\prime}\left(c^{i}\left(\sigma_{t} ; s\right)\right) \pi^{i}(s)} \\
& =\beta_{i}^{T+1} \frac{u_{i}^{\prime}\left(c^{i}\left(\sigma_{T+1}\right)\right)}{u_{i}^{\prime}\left(c^{i}\left(\sigma_{0}\right)\right)} \prod_{t=0}^{T} \frac{\pi^{i}\left(w^{j}\left(\sigma_{t+1}\right)\right)}{q\left(\sigma_{t} ; w^{j}\left(\sigma_{t+1}\right)\right)}
\end{aligned}
$$

Furthermore,

$$
\begin{aligned}
\frac{u_{i}^{\prime}\left(c^{i}\left(\sigma_{t}\right)\right)}{u_{j}^{\prime}\left(c^{j}\left(\sigma_{t}\right)\right)}= & \frac{\beta_{i} \pi^{i}\left(w^{j}\left(\sigma_{t+1}\right)\right) \sum_{s \in w^{j}\left(\sigma_{t+1}\right)} u_{i}^{\prime}\left(c^{i}\left(\sigma_{t} ; s\right)\right) \pi^{i}\left(s \mid w^{j}\left(\sigma_{t+1}\right)\right)}{\beta_{j} \pi^{j}\left(w^{j}\left(\sigma_{t+1}\right)\right) \sum_{s \in w^{j}\left(\sigma_{t+1}\right)} u_{j}^{\prime}\left(c^{j}\left(\sigma_{t} ; s\right)\right) \pi^{j}\left(s \mid w^{j}\left(\sigma_{t+1}\right)\right)} \\
= & \frac{\beta_{i} \pi^{i}\left(w^{j}\left(\sigma_{t+1}\right)\right) \sum_{s \in w^{j}\left(\sigma_{t+1}\right)} \frac{u_{i}^{\prime}\left(c^{i}\left(\sigma_{t} ; s\right)\right)}{u_{i}^{\prime}\left(c^{i}\left(\sigma_{t+1}\right)\right)} \pi^{i}\left(s \mid w^{j}\left(\sigma_{t+1}\right)\right)}{\beta_{j} \pi^{j}\left(w^{j}\left(\sigma_{t+1}\right)\right) \sum_{s \in w^{j}\left(\sigma_{t+1}\right)} \frac{u_{j}^{\prime}\left(c^{j}\left(\sigma_{t} ; s\right)\right)}{u_{j}^{\prime}\left(c^{j}\left(\sigma_{t+1}\right)\right)} \pi^{j}\left(s \mid w^{j}\left(\sigma_{t+1}\right)\right)} \frac{u_{j}^{\prime}\left(c^{j}\left(\sigma_{t+1}\right)\right)}{\left.\left.u_{t+1}\right)\right)} \\
= & \frac{u_{i}^{\prime}\left(c^{i}\left(\sigma_{t+1}\right)\right)}{u_{j}^{\prime}\left(c^{j}\left(\sigma_{t+1}\right)\right)} \frac{\beta_{i}}{\beta_{j}} \frac{\pi^{i}\left(w^{j}\left(\sigma_{t+1}\right)\right)}{\pi^{j}\left(w^{j}\left(\sigma_{t+1}\right)\right)} \frac{\hat{r}_{j}^{j}\left(\sigma_{t}\right)}{\hat{r}_{i}^{j}\left(\sigma_{t}\right)} .
\end{aligned}
$$

It follows that

$$
\frac{u_{i}^{\prime}\left(c^{i}\left(\sigma_{t+1}\right)\right)}{u_{j}^{\prime}\left(c^{j}\left(\sigma_{t+1}\right)\right)}=\frac{u_{i}^{\prime}\left(c^{i}\left(\sigma_{t}\right)\right)}{u_{j}^{\prime}\left(c^{j}\left(\sigma_{t}\right)\right)} \frac{\beta_{j}}{\beta_{i}} \frac{\pi^{j}\left(w^{j}\left(\sigma_{t+1}\right)\right)}{\pi^{i}\left(w^{j}\left(\sigma_{t+1}\right)\right)} \frac{\hat{r}_{i}^{j}\left(\sigma_{t}\right)}{\hat{r}_{j}^{j}\left(\sigma_{t}\right)} .
$$

\section{Proof of Proposition 12}

Using the fact that $\pi$ is i.i.d.,

$$
\lim _{t \rightarrow \infty} \operatorname{Var}_{\pi}\left[\frac{\pi^{j}\left(\sigma_{t} \mid \sigma_{t-1}\right)}{\pi^{i}\left(\sigma_{t} \mid \sigma_{t-1}\right)} \frac{y^{j i}\left(\sigma_{t}\right)}{y^{j i}\left(\sigma_{t-1}\right)} \mid \sigma_{t-1}\right]=0
$$

is equivalent to

$$
\lim _{t \rightarrow \infty}\left[\frac{\pi^{j}(s)}{\pi^{i}(s)} \frac{y^{j i}\left(\sigma_{t-1} ; s\right)}{y^{j i}\left(\sigma_{t-1}\right)}-E_{\pi}\left[\frac{\pi^{j}(\tilde{s})}{\pi^{i}(\tilde{s})} \frac{y^{j i}\left(\sigma_{t-1} ; \tilde{s}\right)}{y^{j i}\left(\sigma_{t-1}\right)} \mid \sigma_{t-1}\right]\right]=0
$$


for all $s \in S$ or, since $\beta_{i}=\beta_{j}$ and by (5),

$$
\begin{gathered}
\lim _{t \rightarrow \infty}\left[\frac{\pi^{j}(s)}{\pi^{i}(s)} \frac{\pi^{i}\left(w_{s}^{j}\right)}{\pi^{j}\left(w_{s}^{j}\right)} \frac{\hat{r}_{j}^{j}\left(\sigma_{t-1} ; s\right)}{\hat{r}_{i}^{j}\left(\sigma_{t-1} ; s\right)}-E_{\pi}\left[\frac{\pi^{j}(\tilde{s})}{\pi^{i}(\tilde{s})} \frac{\pi^{i}\left(w_{\tilde{s}}^{j}\right)}{\pi^{j}\left(w_{\tilde{s}}^{j}\right)} \frac{\hat{r}_{j}^{j}\left(\sigma_{t-1} ; \tilde{s}\right)}{\hat{r}_{i}^{j}\left(\sigma_{t-1} ; \tilde{s}\right)} \mid \sigma_{t-1}\right]\right]=0, \\
\lim _{t \rightarrow \infty}\left[\frac{\pi^{j}\left(s \mid w_{s}^{j}\right)}{\pi^{i}\left(s \mid w_{s}^{j}\right)} \frac{\hat{r}_{j}^{j}\left(\sigma_{t-1} ; s\right)}{\hat{r}_{i}^{j}\left(\sigma_{t-1} ; s\right)}-E_{\pi}\left[\frac{\pi^{j}(\tilde{s})}{\pi^{i}(\tilde{s})} \frac{\pi^{i}\left(w_{\tilde{s}}^{j}\right)}{\pi^{j}\left(w_{\tilde{s}}^{j}\right)} \frac{\hat{r}_{j}^{j}\left(\sigma_{t-1} ; \tilde{s}\right)}{\hat{r}_{i}^{j}\left(\sigma_{t-1} ; \tilde{s}\right)} \mid \sigma_{t-1}\right]\right]=0,
\end{gathered}
$$

where $w_{s}^{j}$ is used to denote the element of $j$ 's partitions containing state $s$.

Denote

$$
\lambda\left(\sigma_{t-1}\right)=E_{\pi}\left[\frac{\pi^{j}(\tilde{s})}{\pi^{i}(\tilde{s})} \frac{\pi^{i}\left(w_{\tilde{s}}^{j}\right)}{\pi^{j}\left(w_{\tilde{s}}^{j}\right)} \frac{\hat{r}_{j}^{j}\left(\sigma_{t-1} ; \tilde{s}\right)}{\hat{r}_{i}^{j}\left(\sigma_{t-1} ; \tilde{s}\right)} \mid \sigma_{t-1}\right] .
$$

For every $\sigma$ and every $\epsilon>0$, there is a $t(\epsilon ; \sigma)$ such that for all $t>t(\epsilon ; \sigma)$ and all $s \in S$,

$$
\begin{aligned}
\left|\frac{\pi^{j}\left(s \mid w_{s}^{j}\right)}{\pi^{i}\left(s \mid w_{s}^{j}\right)} \frac{\hat{r}_{j}^{j}\left(\sigma_{t-1} ; s\right)}{\hat{r}_{i}^{j}\left(\sigma_{t-1} ; s\right)}-\lambda\left(\sigma_{t-1}\right)\right| & <\epsilon \quad \text { or } \\
\left(\lambda\left(\sigma_{t-1}\right)-\epsilon\right) \hat{r}_{i}^{j}\left(\sigma_{t-1} ; s\right) \pi^{i}\left(s \mid w_{s}^{j}\right) & <\pi^{j}\left(s \mid w_{s}^{j}\right) \hat{r}_{j}^{j}\left(\sigma_{t-1} ; s\right) \\
& <\left(\lambda\left(\sigma_{t-1}\right)+\epsilon\right) \hat{r}_{i}^{j}\left(\sigma_{t-1} ; s\right) \pi^{i}\left(s \mid w_{s}^{j}\right) .
\end{aligned}
$$

Thus, for any $w^{j}$, summing over $s \in w^{j}$, we obtain

$$
\begin{aligned}
\left(\lambda\left(\sigma_{t-1}\right)-\epsilon\right) \sum_{s \in w^{j}} \hat{r}_{i}^{j}\left(\sigma_{t-1} ; s\right) \pi^{i}\left(s \mid w^{j}\right) & <\sum_{s \in w^{j}} \pi^{j}\left(s \mid w^{j}\right) \hat{r}_{j}^{j}\left(\sigma_{t-1} ; s\right) \\
& <\left(\lambda\left(\sigma_{t-1}\right)+\epsilon\right) \sum_{s \in w^{j}} \hat{r}_{i}^{j}\left(\sigma_{t-1} ; s\right) \pi^{i}\left(s \mid w^{j}\right)
\end{aligned}
$$

and since by the definition of $\hat{r}_{i}^{j}, \sum_{s \in w^{j}} \hat{r}_{i}^{j}\left(\sigma_{t-1} ; s\right) \pi^{i}\left(s \mid w^{j}\right)=1$ and $\sum_{s \in w^{j}} \pi^{j}\left(s \mid w^{j}\right) \hat{r}_{j}^{j}\left(\sigma_{t-1}\right.$; $s)=1$,

$$
\left(\lambda\left(\sigma_{t-1}\right)-\epsilon\right) \pi^{i}\left(w^{j}\right)<\pi^{j}\left(w^{j}\right)<\left(\lambda\left(\sigma_{t-1}\right)+\epsilon\right) \pi^{i}\left(w^{j}\right) .
$$

Finally, summing once again over all $w^{j}, \lambda\left(\sigma_{t-1}\right)-\epsilon<1<\lambda\left(\sigma_{t-1}\right)+\epsilon$ or $\lim _{t \rightarrow \infty} \lambda\left(\sigma_{t}\right)=1$. It follows by equations (19), (20), and (21) that

$$
\begin{aligned}
\lim _{t \rightarrow \infty} \frac{\pi^{j}\left(\sigma_{t} \mid \sigma_{t-1}\right)}{\pi^{i}\left(\sigma_{t} \mid \sigma_{t-1}\right)} \frac{y^{j i}\left(\sigma_{t}\right)}{y^{j i}\left(\sigma_{t-1}\right)} & =1 \quad \pi \text {-a.s. on } \sigma \text { satisfying } \\
\lim _{t \rightarrow \infty} \operatorname{Var}_{\pi}\left[\frac{\pi^{j}\left(\sigma_{t} \mid \sigma_{t-1}\right)}{\pi^{i}\left(\sigma_{t} \mid \sigma_{t-1}\right)} \frac{y^{j i}\left(\sigma_{t}\right)}{y^{j i}\left(\sigma_{t-1}\right)} \mid \sigma_{t-1}\right] & =0 .
\end{aligned}
$$

We next prove a lemma that is analogous to Proposition 3 in BC (2010) and that characterizes equilibrium prices in an i.i.d. economy with two agents on a path on which one of the agents disappears. This lemma will be subsequently used in the proofs of Propositions 13 and 14.

In the case of two agents, even when financial constraints are different, both agents can effectively trade on those events that are measurable w.r.t. both partitions. We can thus 
denote the finest common coarsening of $W^{i}$ and $W^{j}$ by $W$ and consider an incomplete market with generalized unit securities paying on $w \in W$.

LEMmA 17: Consider an IDC equilibrium. Let $I=\{i ; j\}$. Assume that the initial endowment of the economy is i.i.d. Let $J=\left\{\sigma \in \Omega \mid \lim _{t \rightarrow \infty} c^{j}\left(\sigma_{t}\right)=0\right\}$ be the set of paths on which $j$ vanishes. Let $W$ be the finest common coarsening of $W^{i}$ and $W^{j}$. Then, $\pi$-a.s. on J, for $w \in W$,

$$
\lim _{t \rightarrow \infty} q\left(\sigma_{t} ; w\right)=q^{i}\left(s\left(\sigma_{t}\right) ; w\right)=: \frac{\beta^{i} \pi^{i}(w) \sum_{\tilde{s} \in w^{j}} u_{i}^{\prime}(e(\tilde{s})) \pi^{i}(\tilde{s} \mid w)}{u_{i}^{\prime}\left(e\left(s\left(\sigma_{t}\right)\right)\right)} .
$$

PROOF: For $\epsilon>0$, define the set

$$
J_{\epsilon}=:\left\{\left\{\sigma|| q\left(\sigma_{t} ; w\right)-q^{i}\left(s\left(\sigma_{t}\right) ; w\right) \mid>\epsilon\right\} \text { i.o. }\right\} \cap J .
$$

On $\sigma \in J, c^{i}\left(\sigma_{t}\right) \rightarrow e\left(s\left(\sigma_{t}\right)\right)$ and so there exists a $T(\sigma)$ such that if $t \geq T(\sigma)$, then

$$
\frac{1-\frac{1}{2} \frac{\epsilon}{\beta_{i}} \frac{u_{i}^{\prime}\left(m^{\prime}\right)}{u_{i}^{\prime}(\tilde{m})}}{1-\frac{\epsilon}{\beta_{i}} \frac{u_{i}^{\prime}\left(m^{\prime}\right)}{u_{i}^{\prime}(\tilde{m})}} \geq \frac{u_{i}^{\prime}\left(c^{i}\left(\sigma_{t}\right)\right)}{u_{i}^{\prime}\left(e\left(s\left(\sigma_{t}\right)\right)\right)} \geq 1 .
$$

Note that

$$
q\left(\sigma_{t} ; w\right)-q^{i}\left(s\left(\sigma_{t}\right) ; w\right)=\beta_{i} \sum_{s \in w^{j}} \pi^{i}(s)\left[\frac{u_{i}^{\prime}\left(c^{i}\left(\sigma_{t} ; s\right)\right)}{u_{i}^{\prime}\left(c^{i}\left(\sigma_{t}\right)\right)}-\frac{u_{i}^{\prime}(e(s))}{u_{i}^{\prime}\left(e\left(s\left(\sigma_{t}\right)\right)\right)}\right]
$$

and, hence, (22) combined with the fact that $\pi^{i}(s)>\underline{\pi}>0$ for all $s \in S$ implies that there is a subsequence $\left\{t_{k}\right\}_{k=1}^{\infty}$ with $t_{1} \geq T(\sigma)$ such that either

$$
\begin{aligned}
& \pi^{i}\left(s \mid \frac{u_{i}^{\prime}\left(c^{i}\left(\sigma_{t_{k}} ; s\right)\right)}{u_{i}^{\prime}\left(c^{i}\left(\sigma_{t_{k}}\right)\right)}-\frac{u_{i}^{\prime}(e(s))}{u_{i}^{\prime}\left(e\left(s\left(\sigma_{t_{k}}\right)\right)\right)}>\frac{\epsilon}{\beta_{i}}\right)>\underline{\pi} \quad \text { and, hence, } \\
& \pi\left(s \mid \frac{u_{i}^{\prime}\left(c^{i}\left(\sigma_{t_{k}} ; s\right)\right)}{u_{i}^{\prime}\left(c^{i}\left(\sigma_{t_{k}}\right)\right)}-\frac{u_{i}^{\prime}(e(s))}{u_{i}^{\prime}\left(e\left(s\left(\sigma_{t_{k}}\right)\right)\right)}>\frac{\epsilon}{\beta_{i}}\right)>\underline{\pi}
\end{aligned}
$$

for all $k \geq 1$ or

$$
\begin{aligned}
& \pi^{i}\left(s \mid \frac{u_{i}^{\prime}\left(c^{i}\left(\sigma_{t_{k}} ; s\right)\right)}{u_{i}^{\prime}\left(c^{i}\left(\sigma_{t_{k}}\right)\right)}-\frac{u_{i}^{\prime}(e(s))}{u_{i}^{\prime}\left(e\left(s\left(\sigma_{t_{k}}\right)\right)\right)}<-\frac{\epsilon}{\beta_{i}}\right)>\underline{\pi} \quad \text { and, hence, } \\
& \pi\left(s \mid \frac{u_{i}^{\prime}\left(c^{i}\left(\sigma_{t_{k}} ; s\right)\right)}{u_{i}^{\prime}\left(c^{i}\left(\sigma_{t_{k}}\right)\right)}-\frac{u_{i}^{\prime}(e(s))}{u_{i}^{\prime}\left(e\left(s\left(\sigma_{t_{k}}\right)\right)\right)}<-\frac{\epsilon}{\beta_{i}}\right)>\underline{\pi}
\end{aligned}
$$

for all $k \geq 1$. 
Suppose (23) holds for all $k \geq 1$. Then there is a $\sigma^{\prime}$ such that $\sigma_{t}^{\prime}=\sigma_{t}$ for all $t \leq t_{k}$ such that

$$
\begin{aligned}
\frac{u_{i}^{\prime}\left(c^{i}\left(\sigma_{t_{k}+1}^{\prime}\right)\right)}{u_{i}^{\prime}\left(c^{i}\left(\sigma_{t}^{\prime}\right)\right)}-\frac{u_{i}^{\prime}\left(e\left(s\left(\sigma_{t_{k}+1}^{\prime}\right)\right)\right)}{u_{i}^{\prime}\left(e\left(s\left(\sigma_{t}^{\prime}\right)\right)\right)} & >\frac{\epsilon}{\beta_{i}}, \\
\frac{u_{i}^{\prime}\left(c^{i}\left(\sigma_{t_{k}+1}^{\prime}\right)\right)}{u_{i}^{\prime}\left(c^{i}\left(\sigma_{t}^{\prime}\right)\right)} & >\frac{u_{i}^{\prime}\left(e\left(s\left(\sigma_{t_{k}+1}^{\prime}\right)\right)\right)}{u_{i}^{\prime}\left(e\left(s\left(\sigma_{t}^{\prime}\right)\right)\right)}\left(\frac{u_{i}^{\prime}\left(e\left(s\left(\sigma_{t}^{\prime}\right)\right)\right)}{u_{i}^{\prime}\left(e\left(s\left(\sigma_{t_{k}+1}^{\prime}\right)\right)\right)} \frac{\epsilon}{\beta_{i}}+1\right), \\
\frac{u_{i}^{\prime}\left(c^{i}\left(\sigma_{t_{k}+1}^{\prime}\right)\right)}{u_{i}^{\prime}\left(e\left(s\left(\sigma_{t_{k}+1}^{\prime}\right)\right)\right)} & >\underbrace{\frac{u_{i}^{\prime}\left(c^{i}\left(\sigma_{t}^{\prime}\right)\right)}{u_{i}^{\prime}\left(e\left(s\left(\sigma_{t}^{\prime}\right)\right)\right)}}_{\geq 1} \underbrace{\frac{u_{i}^{\prime}\left(e\left(s\left(\sigma_{t}^{\prime}\right)\right)\right)}{u_{i}^{\prime}\left(e\left(s\left(\sigma_{t^{\prime}+1}^{\prime}\right)\right)\right.}}_{\geq \frac{u_{i}^{\prime}\left(m^{\prime}\right)}{u_{i}^{\prime}(\tilde{m})}} \frac{\epsilon}{\beta_{i}}+1) \\
& \geq \frac{u_{i}^{\prime}\left(m^{\prime}\right)}{u_{i}^{\prime}(\tilde{m})} \frac{\epsilon}{\beta_{i}}+1 .
\end{aligned}
$$

Letting $\epsilon^{\prime}=\frac{1}{2} \frac{u_{i}^{\prime}\left(m^{\prime}\right)}{u_{i}^{\prime}(\tilde{m})} \frac{\epsilon}{\beta_{i}}>0$, we obtain

$$
\pi\left(s \mid \frac{u_{i}^{\prime}\left(c^{i}\left(\sigma_{t_{k}} ; s\right)\right)}{u_{i}^{\prime}(e(s))} \geq 1+\epsilon^{\prime}\right)>\underline{\pi} \quad \text { for all } k \geq 1 \text { on } \sigma \in J_{\epsilon} .
$$

Symmetrically, if (24) holds, then one obtains

$$
\pi\left(s \mid \frac{u_{i}^{\prime}\left(c^{i}\left(\sigma_{t_{k}} ; s\right)\right)}{u_{i}^{\prime}(e(s))} \leq 1-\epsilon^{\prime}\right)>\underline{\pi} \quad \text { for all } k \geq 1 \text { on } \sigma \in J_{\epsilon} .
$$

Hence, for each $\sigma \in J_{\epsilon}$, either

$$
\sum_{t=0}^{\infty} \pi\left(s \mid \frac{u_{i}^{\prime}\left(c^{i}\left(\sigma_{t} ; s\right)\right)}{u_{i}^{\prime}(e(s))} \geq 1+\epsilon^{\prime}\right) \geq \sum_{k=1}^{\infty} \pi\left(s \mid \frac{u_{i}^{\prime}\left(c^{i}\left(\sigma_{t_{k}} ; s\right)\right)}{u_{i}^{\prime}(e(s))} \geq 1+\epsilon^{\prime}\right)=\infty
$$

or

$$
\sum_{t=0}^{\infty} \pi\left(s \mid \frac{u_{i}^{\prime}\left(c^{i}\left(\sigma_{t} ; s\right)\right)}{u_{i}^{\prime}(e(s))} \leq 1-\epsilon^{\prime}\right) \geq \sum_{k=1}^{\infty} \pi\left(s \mid \frac{u_{i}^{\prime}\left(c^{i}\left(\sigma_{t_{k}} ; s\right)\right)}{u_{i}^{\prime}(e(s))} \leq 1-\epsilon^{\prime}\right)=\infty,
$$

which, by the EBC lemma in BC (2010) implies

$$
\sigma \in\left\{\left\{\tilde{\sigma} \mid \frac{u_{i}^{\prime}\left(c^{i}\left(\sigma_{t}\right)\right)}{u_{i}^{\prime}\left(e\left(s\left(\sigma_{t}\right)\right)\right)} \leq 1-\epsilon^{\prime}\right\} \text { i.o. }\right\} \cup\left\{\left\{\tilde{\sigma} \mid \frac{u_{i}^{\prime}\left(c^{i}\left(\sigma_{t}\right)\right)}{u_{i}^{\prime}\left(e\left(s\left(\sigma_{t}\right)\right)\right)} \geq 1+\epsilon^{\prime}\right\} \text { i.o. }\right\},
$$

and since $u_{i}^{\prime}$ is continuous, $c^{i}\left(\sigma_{t}\right) \nrightarrow e\left(\sigma_{t}\right), \pi$-a.s. Since $J_{\epsilon} \subseteq J$, it follows that $\pi\left(J_{\epsilon}\right)=0$. 


\section{Proof of Proposition 13}

Extrapolating (5) over $t$, we obtain

$$
\lim _{T \rightarrow \infty} \frac{1}{T} \ln \frac{u_{j}^{\prime}\left(\sigma_{T}\right)}{u_{i}^{\prime}\left(\sigma_{T}\right)}=\ln \frac{\beta_{i}}{\beta_{j}}+\sum_{w^{j} \in W^{j}} \pi\left(w^{j}\right) \ln \frac{\pi^{i}\left(w^{j}\right)}{\pi^{j}\left(w^{j}\right)}+\lim _{T \rightarrow \infty} \frac{1}{T} \sum_{t=1}^{T} \ln \frac{\hat{r}_{j}^{j}\left(\sigma_{t}\right)}{\hat{r}_{i}^{j}\left(\sigma_{t}\right)} .
$$

If $\hat{r}_{j}^{j}\left(\sigma_{t}\right)=1$ for all $\sigma_{t}$, then

$$
\lim _{T \rightarrow \infty} \frac{1}{T} \sum_{t=1}^{T} \ln \frac{\hat{r}_{j}^{j}\left(\sigma_{t}\right)}{\hat{r}_{i}^{j}\left(\sigma_{t}\right)}=\lim _{T \rightarrow \infty} \frac{1}{T} \sum_{t=1}^{T} \ln \frac{\sum_{s \in w^{j}\left(\sigma_{t+1}\right)} u_{i}^{\prime}\left(c^{i}\left(\sigma_{t} ; s\right)\right) \pi^{i}\left(s \mid w^{j}\left(\sigma_{t+1}\right)\right)}{u_{i}^{\prime}\left(c^{i}\left(\sigma_{t+1}\right)\right)} .
$$

(a) In particular, if as in (a), $\beta_{i}=\beta_{j}$ and $\pi^{i}=\pi^{j}=\pi$, then in expectations,

$$
\begin{gathered}
-E_{\pi}\left(\ln \frac{u_{i}^{\prime}\left(c^{i}\left(\sigma_{t+1}\right)\right)}{\sum_{s \in w^{j}\left(\sigma_{t+1}\right)} u_{i}^{\prime}\left(c^{i}\left(\sigma_{t} ; s\right)\right) \pi\left(s \mid w^{j}\left(\sigma_{t+1}\right)\right)}\right) \\
\geq-\ln E_{\pi} \frac{u_{i}^{\prime}\left(c^{i}\left(\sigma_{t+1}\right)\right)}{\sum_{s \in w^{j}\left(\sigma_{t+1}\right)} u_{i}^{\prime}\left(c^{i}\left(\sigma_{t} ; s\right)\right) \pi\left(s \mid w^{j}\left(\sigma_{t+1}\right)\right)}=0,
\end{gathered}
$$

and since the economy faces aggregate risk, there is at least one $\tilde{w}^{j}$ such that the inequality is strict whenever $w^{j}\left(\sigma_{t+1}\right)=\widetilde{w}^{j}$ is as in the statement of the proposition. Since $\tilde{w}^{j}$ occurs i.o. $\pi$-a.s. on every path $\sigma$, it follows that a.s.

$$
\lim _{T \rightarrow \infty} \frac{1}{T} \sum_{t=1}^{T} \ln \frac{\hat{r}_{j}^{j}\left(\sigma_{t}\right)}{\hat{r}_{i}^{j}\left(\sigma_{t}\right)}>0
$$

and, hence, a.s.

$$
\lim _{T \rightarrow \infty} \frac{1}{T} \ln \frac{u_{j}^{\prime}\left(c^{j}\left(\sigma_{T}\right)\right)}{u_{i}^{\prime}\left(c^{i}\left(\sigma_{T}\right)\right)}>\ln \frac{\beta_{i}}{\beta_{j}}+\sum_{w^{j} \in W^{j}} \pi\left(w^{j}\right) \ln \frac{\pi^{i}\left(w^{j}\right)}{\pi^{j}\left(w^{j}\right)}=0 .
$$

However, $\lim _{T \rightarrow \infty} \frac{1}{T} \ln \frac{u_{j}^{\prime}\left(c^{j}\left(\sigma_{T}\right)\right)}{u_{i}^{\prime}\left(c^{l}\left(\sigma_{T}\right)\right)}>0$ can only obtain if $u_{j}^{\prime}\left(c^{j}\left(\sigma_{t}\right)\right) \rightarrow \infty$ or if $c^{j}\left(\sigma_{t}\right) \rightarrow 0$. It follows that in this case $j$ a.s. vanishes.

(b) If $i$ and $j$ are the only agents in the economy, then Lemma 17 above implies that on the set of paths on which $j$ vanishes, asset prices converge to equilibrium prices in an economy with a representative agent $i$. Hence, $i$ 's consumption converges toward the total endowment of the economy, not just on the path on which $j$ vanishes, $\sigma_{t}$ as $t \rightarrow \infty$, 
but also on $\left(\sigma_{t} ; s\right)$ for any $s \in S$ as $t \rightarrow \infty$. This in turn implies

$$
\begin{aligned}
\lim _{T \rightarrow \infty} \frac{1}{T} \sum_{t=1}^{T} \ln \frac{\hat{r}_{j}^{j}\left(\sigma_{t}\right)}{\hat{r}_{i}^{j}\left(\sigma_{t}\right)} & =\lim _{T \rightarrow \infty} \frac{1}{T} \sum_{t=1}^{T} \ln \frac{\sum_{s \in w^{j}\left(\sigma_{t+1}\right)} u_{i}^{\prime}\left(c^{i}\left(\sigma_{t} ; s\right)\right) \pi^{i}\left(s \mid w^{j}\left(\sigma_{t+1}\right)\right)}{u_{i}^{\prime}\left(c^{i}\left(\sigma_{t+1}\right)\right)} \\
& =\sum_{s \in S} \pi(s) \ln \frac{\sum_{s \in w_{s}^{j}} u_{i}^{\prime}(e(s)) \pi^{i}\left(s \mid w_{s}^{j}\right)}{u_{i}^{\prime}(e(s))} .
\end{aligned}
$$

Hence, the condition for $j$ not to vanish provided that he is fully insured across the states within the elements of his partition $W^{j}$ is

$$
\ln \frac{\beta_{i}}{\beta_{j}}+\sum_{w^{j} \in W^{j}} \pi\left(w^{j}\right) \ln \frac{\pi^{i}\left(w^{j}\right)}{\pi^{j}\left(w^{j}\right)}<\sum_{s \in S} \pi(s) \ln \frac{\sum_{s \in w_{s}^{j}} u_{i}^{\prime}(e(s)) \pi^{i}\left(s \mid w_{s}^{j}\right)}{u_{i}^{\prime}(e(s))} .
$$

\section{Proof of Proposition 14}

As above, let $W$ denote the finest common coarsening of $W^{i}$ and $W^{j}$, that is, the set of tradable events. By the assumption on $W^{j}$, there is an element $\bar{w} \in W$ with two distinct states $s_{\bar{w}}$ and $s_{\bar{w}}^{\prime}$. Without loss of generality, we show the result for $j$; the same argument applies for agent $i$.

LEMMA 18: Under the assumptions of Proposition 14, on the set of paths, on which $j$ vanishes, asset prices satisfy

$$
\lim _{t \rightarrow \infty} q\left(\sigma_{t} ; w\right)=q^{i}(s ; w)=\beta_{i} \frac{\sum_{\tilde{s} \in w} u_{i}^{\prime}(e(\tilde{s})) \pi^{i}(\tilde{s})}{u_{i}^{\prime}(e(s))}
$$

and $\sum_{w \in W} q^{i}(s ; w) \leq 1$ for any $s \in S$.

PROOF: The convergence result follows from Lemma 17. Since $\beta_{i}<\frac{u_{i}^{\prime}\left(m^{\prime}\right)}{u_{i}^{\prime}(\tilde{m})}$, it follows that for any $s \in S$,

$$
\sum_{w \in W} q^{i}(s ; w)=\beta_{i} \frac{\sum_{\tilde{s} \in S} u_{i}^{\prime}(e(\tilde{s})) \pi^{i}(\tilde{s})}{u_{i}^{\prime}(e(s))}<\frac{u_{i}^{\prime}\left(m^{\prime}\right)}{u_{i}^{\prime}(\tilde{m})} \frac{\sum_{\tilde{s} \in S} u_{i}^{\prime}(e(\tilde{s})) \pi^{i}(\tilde{s})}{u_{i}^{\prime}(e(s))} \leq 1 .
$$

For each $w \in W$, choose an $s_{w} \in w$ such that $s_{\bar{w}}$ is the relevant state for $\bar{w}$. Define $\left(\theta^{*}(w)\right)_{w \in W}$ as the solution to the system of equations

$$
\theta^{*}(w)+e^{j}\left(s_{w}\right)=\sum_{\tilde{w} \in W} \theta^{*}(\tilde{w}) q^{i}\left(s_{w} ; \tilde{w}\right) \quad \text { for all } s_{w}, w \in W .
$$

Note that a solution exists and is unique except on a measure- 0 set of parameters of the economy. 
Furthermore, except for a set of initial endowments with measure 0 ,

$$
\sum_{\tilde{w}} \theta^{*}(\tilde{w}) q^{i}\left(s_{\bar{w}} ; \tilde{w}\right)-e^{j}\left(s_{\bar{w}}\right)-\sum_{\tilde{w}} \theta^{*}(\tilde{w}) q^{i}\left(s_{\tilde{w}}^{\prime} ; \tilde{w}\right)+e^{j}\left(s_{\tilde{w}}^{\prime}\right)=\hat{d} \neq 0 .
$$

Let $d>0$. Choose

$$
\varepsilon(d)=\min \left\{\frac{d}{2}\left(1-\max _{s \in S} \sum_{w \in W} q^{i}(s ; w)\right) ; \frac{1-\max _{s \in S} \sum_{w \in W} q^{i}(s ; w)}{4} 8 N|W|\right\} .
$$

Let $\xi(d)=\underline{\pi}^{\bar{k}(d)}$, where $\bar{k}(d)$ is the smallest positive integer such that

$$
\begin{aligned}
& {\left[\min _{s \in S} \frac{1}{\sum_{w \in W} q^{i}(s ; w)+\frac{1-\max _{s \in S} \sum_{w \in W} q^{i}(s ; w)}{2}}\right]^{\bar{k}}} \\
& >\max \left\{\frac{N-\min _{w \in W} \theta^{*}(w)}{d-\varepsilon(d)} ; \frac{N+\min _{w \in W} \theta^{*}(w)}{d-\varepsilon(d)}\right\} .
\end{aligned}
$$

Suppose in a manner of contradiction that $j$ vanishes a.s. conditional on node $\bar{\sigma}_{\bar{t}}$. Then there exists a $\hat{t}(\varepsilon(d) ; \xi(d))=\hat{t}(d)$ such that

$$
\begin{aligned}
& \pi\left(\sigma \mid \sigma_{\bar{t}}=\bar{\sigma}_{\bar{t}}, c^{j}\left(\sigma_{t}\right)<\frac{\varepsilon(d)}{2}\right. \text { and } \\
& \left.\quad\left|q\left(\sigma_{t} ; w\right)-q^{i}\left(s\left(\sigma_{t}\right) ; w\right)\right|<\frac{\varepsilon(d)}{8 N\left|W^{j}\right|} \text { for all } t \geq \hat{t}(d)\right) \\
& \quad>1-\xi(d) .
\end{aligned}
$$

The condition on $q\left(\sigma_{t} ; w\right)$ together with those on $\varepsilon(d)$ imply that for any $\theta\left(\sigma_{t} ; w\right) \in$ $[-N ; N]^{|W|}$ (including $\theta\left(\sigma_{t} ; w\right)=\theta^{*}(w)$ ) and for all $t \geq \hat{t}(d)$ on the set of paths in the set on the l.h.s. of (27),

$$
\left|\sum_{w \in W} \theta\left(\sigma_{t} ; w\right) q\left(\sigma_{t} ; w\right)-\sum_{w \in W} \theta\left(\sigma_{t} ; w\right) q^{i}(s ; w)\right|<\frac{\varepsilon(d)}{4} .
$$


Since for any $\tilde{t}>\hat{t}(d)$, the number of nodes $\sigma_{\tilde{t}}$ such that $\sigma_{\bar{t}}=\bar{\sigma}_{\bar{t}}$ is finite, it follows that there is at least one node $\tilde{\sigma}_{\tilde{t}}$ such that

$$
\begin{aligned}
& \pi\left(\Xi\left(\tilde{\sigma}_{\tilde{t}} ; d\right)\right) \\
&=\pi\left(\sigma \mid \sigma_{\bar{t}}=\bar{\sigma}_{\bar{t}}, \sigma_{\tilde{t}}=\tilde{\sigma}_{\tilde{t}}, c^{j}\left(\sigma_{t}\right)<\frac{\varepsilon(d)}{2}\right. \text { and } \\
&\left|\sum_{w \in W} \theta\left(\sigma_{t} ; w\right) q\left(\sigma_{t} ; w\right)-\sum_{w \in W} \theta\left(\sigma_{t} ; w\right) q^{i}(s ; w)\right|<\frac{\varepsilon(d)}{4} \\
&\text { for all } t \geq \tilde{t}) \\
&>1-\xi(d) .
\end{aligned}
$$

Denote by $\tilde{\Omega}(d)$ the set of all nodes $\tilde{\sigma}_{\tilde{t}}$ satisfying these properties. Note that for any given path $\sigma$, on which $j$ vanishes, $\sigma_{t} \in \tilde{\Omega}(d)$ occurs i.o. Furthermore, if $d^{\prime}<d$, then $\varepsilon\left(d^{\prime}\right) \leq \varepsilon(d)$ and $\xi\left(d^{\prime}\right) \leq \xi(d)$, so that $\tilde{\Omega}\left(d^{\prime}\right) \subseteq \tilde{\Omega}(d)$.

Q.E.D.

LEMMA 19: Almost surely, on every path $\sigma$ with $\sigma_{\bar{t}}=\bar{\sigma}_{\bar{t}},\left|\theta_{j}\left(\sigma_{t} ; \bar{w}\right)-\theta^{*}(\bar{w})\right|<d$ for every $\sigma_{t} \in \tilde{\Omega}(d)$.

Proof: Given $\tilde{\sigma}_{\tilde{t}} \in \tilde{\Omega}(d)$, consider its continuations $\sigma$ with $\sigma_{\tilde{t}}=\tilde{\sigma}_{\tilde{t}}$ that belong to $\Xi\left(\tilde{\sigma}_{\tilde{t}} ; d\right)$ in $(29)$. Note that $w\left(\sigma_{\tilde{t}+1}\right)=\bar{w}$ occurs with probability at least $\underline{\pi}$. Suppose first in a manner of contradiction that $\theta_{j}\left(\sigma_{\tilde{t}} ; \bar{w}\right) \geq d+\theta^{*}(\bar{w})$ and $s_{w^{1}}=s_{\bar{w}}=s\left(\sigma_{\tilde{t}+1}\right)$. The budget constraint of $j$ together with the definition of $\left(\theta^{*}(w)\right)_{w \in W}$ in (25) implies that

$$
\sum_{w \in W} \theta_{j}\left(\sigma_{\tilde{t}} ; s_{w^{1}} ; w\right) q\left(\sigma_{\tilde{t}} ; s_{w^{1}} ; w\right) \geq d-\frac{\varepsilon(d)}{2}+\sum_{w \in W} \theta^{*}(w) q^{i}\left(s_{w^{1}} ; w\right)
$$

and, hence, by (28),

$$
\sum_{w \in W} \theta_{j}\left(\sigma_{\tilde{t}} ; s_{w^{1}} ; w\right) q\left(\sigma_{\tilde{t}} ; s_{w^{1}} ; w\right) \geq d-\varepsilon(d)+\sum_{w \in W} \theta^{*}(w) q\left(\sigma_{t-1} ; s_{w^{1}} ; w\right) .
$$

Since by the definition of $\varepsilon(d), d>2 \varepsilon(d)$ and

$$
\sum_{w \in W} q\left(\sigma_{\tilde{t}} ; s_{w^{1}} ; w\right)<\sum_{w \in W} q^{i}\left(s_{w^{1}} ; w\right)+\frac{1-\max _{s \in S} \sum_{w \in W} q^{i}(s ; w)}{2}<1,
$$

we have that for at least one $w^{2} \in W^{j}$,

$$
\begin{aligned}
\theta_{j}\left(\sigma_{\tilde{t}} ; s_{w^{1}} ; w^{2}\right) & \geq \theta^{*}\left(w^{2}\right)+\frac{d-\varepsilon(d)}{\sum_{w \in W} q\left(\sigma_{\tilde{t}} ; s_{w^{1}} ; w\right)} \\
& >\theta^{*}\left(w^{2}\right)+\varepsilon(d) .
\end{aligned}
$$


Recursively, noting that by assumption, for every $\kappa$,

$$
\begin{aligned}
d & >\frac{\varepsilon(d)}{1-\max _{s \in S} \sum_{w \in W} q^{i}(s ; w)} \\
& \geq \varepsilon(d)+\max _{s \in S} \sum_{w \in W} q^{i}(s ; w) \varepsilon(d)+\cdots+\left(\max _{s \in S} \sum_{w \in W} q^{i}(s ; w)\right)^{\kappa} \varepsilon(d)>\varepsilon(d),
\end{aligned}
$$

there is a sequence of $\bar{k}(d)$ states such that

$$
\begin{aligned}
& \theta_{j}\left(\sigma_{\tilde{t}} ; s_{w^{1}} \ldots s_{w^{\bar{k}}} ; w^{\bar{k}(d)+1}\right) \\
& \geq \theta^{*}\left(w^{\bar{k}(d)+1}\right) \\
& \frac{\frac{d-\varepsilon(d)}{\sum_{w \in W} q\left(\sigma_{\tilde{t}} ; s_{w^{1}} ; w\right)}-\varepsilon(d)}{\sum_{w \in W} q\left(\sigma_{\tilde{t}} ; s_{w^{1}} ; s_{w^{2}} ; w\right)}-\varepsilon(d) \\
& +\frac{\frac{\sum_{w \in W}}{\ldots}-\varepsilon(d) \ldots}{\sum_{w \in W} q\left(\sigma_{\tilde{t}} ; s_{w^{1}} ; s_{w^{2}} ; \ldots s_{w^{k}} ; w\right)}-\varepsilon(d) \\
& \geq \min _{w \in W} \theta^{*}(w)+\frac{d-\varepsilon(d)}{\prod_{\kappa=1}^{\bar{k}}\left[\sum_{w \in W} q^{i}\left(s_{w^{k}} ; w\right)+\frac{1-\max _{s \in S} \sum_{w \in W} q^{i}(s ; w)}{2}\right]}
\end{aligned}
$$

$>N$.

Note, furthermore, that by assumption, the probability of such a sequence occurring conditional on $\tilde{\sigma}_{\bar{t}}$ is at least $\pi\left(s_{w^{1}} \ldots s_{w^{\bar{k}(d)}}\right) \geq \underline{\pi}^{\bar{k}(d)}=\xi(d)>0$ and, hence, the choice of $\sigma$ with $\sigma_{\tilde{t}+\bar{k}(d)}=\left(\tilde{\sigma}_{\tilde{t}} ; s_{w^{1}} \ldots s_{w^{\bar{k}(d)}}\right)$ belonging to the set $\Xi\left(\tilde{\sigma}_{\tilde{t}} ; d\right)$ is nonvacuous. Furthermore, $\theta_{j}\left(\tilde{\sigma}_{i} ; s_{w^{1}} \ldots s_{w^{\bar{k}(d)}} ; w\right)>N$ for some $w \in W$, in contradiction to Lemma 2 .

A symmetric argument shows that if $\theta_{j}\left(\tilde{\sigma}_{\bar{t}} ; \bar{w}\right) \leq-d+\theta^{*}(\bar{w})$ for some $\tilde{\sigma}_{\bar{t}} \in \tilde{\Omega}(d)$, there is a path $\sigma$ with $\sigma_{\tilde{t}}=\tilde{\sigma}_{\tilde{t}}$ such that $\sigma_{\tilde{t}+\bar{k}(d)}=\left(\tilde{\sigma}_{\tilde{t}} ; s_{w^{1}} \ldots s_{w^{k}(d)}\right)$ has probability larger than $\xi(d)$ and, thus, belongs to $\Xi\left(\tilde{\sigma}_{\tilde{t}} ; d\right)$ and $\theta_{j}\left(\tilde{\sigma}_{\tilde{t}} ; s_{w^{1}} \ldots s_{w^{\bar{k}(d)}} ; w\right)<-N$ for some $w \in W$, in contradiction to Lemma 2

It follows that a.s. on every path $\sigma$ with $\sigma_{\bar{t}}=\bar{\sigma}_{\bar{t}},\left|\theta_{j}\left(\sigma_{t} ; \bar{w}\right)-\theta^{*}(\bar{w})\right|<d$ holds for any $\sigma_{t} \in \tilde{\Omega}(d)$, establishing the result of the lemma.

To conclude the proof of Proposition 14, we now establish that if $\left|\theta_{j}\left(\sigma_{t} ; \bar{w}\right)-\theta^{*}(\bar{w})\right|<\frac{\hat{d}}{4}$ for some $\sigma_{t} \in \tilde{\Omega}\left(\frac{\hat{d}}{4}\right)$, then the subsequent occurrence of state $s_{\bar{w}}^{\prime}$ implies that $j$ 's asset holdings will violate Lemma 2 in finite time with strictly positive probability. This generates the necessary contradiction. 
Indeed, let $d=\frac{\hat{d}}{4}$. By Lemma 19, there is a finite period $t_{0}\left(\frac{\hat{d}}{4}\right)$ such that

$$
\begin{aligned}
& \pi\left(\tilde{\Xi}\left(\tilde{\sigma}_{\tilde{t}}\right)\right) \\
& =\pi|\sigma| \sigma_{\bar{t}}=\bar{\sigma}_{\bar{t}}, c^{j}\left(\sigma_{t}\right)<\frac{\varepsilon\left(\frac{\hat{d}}{4}\right)}{2}, \\
& \quad\left|\sum_{w \in W} \theta(w) q\left(\sigma_{t} ; w\right)-\sum_{w \in W} \theta^{*}(w) q^{i}(s ; w)\right|<\frac{\varepsilon\left(\frac{\hat{d}}{4}\right)}{4} \\
& \quad \text { and, for } \sigma_{t} \in \tilde{\Omega}\left(\frac{\hat{d}}{4}\right),\left|\theta_{j}\left(\sigma_{t} ; \bar{w}\right)-\theta^{*}(\bar{w})\right|<\frac{\hat{d}}{4} \\
& \left.\left.\quad \text { for all } t \geq t_{0}\left(\frac{\hat{d}}{4}\right)\right)\right) \\
& >1-\xi\left(\frac{\hat{d}}{4}\right) .
\end{aligned}
$$

As above, for any $\tilde{t}>t_{0}\left(\frac{\hat{d}}{4}\right)$, there exists at least one node $\tilde{\sigma}_{\tilde{t}} \in \tilde{\Omega}\left(\frac{\hat{d}}{4}\right)$ such that $\mid \theta_{j}\left(\tilde{\sigma}_{\tilde{t}} ; \bar{w}\right)-$ $\theta^{*}(\bar{w}) \mid<\frac{\hat{d}}{4}$. Note that the state $s_{\bar{w}}^{\prime}$ satisfying condition (26) occurs with a probability of at least $\underline{\pi}$ conditional on $\tilde{\sigma}_{\bar{t}}$, and, hence, combining the budget constraint of $j$ at $\left(\tilde{\sigma}_{\tilde{t}} ; s_{w^{1}}=\right.$ $\left.s_{\tilde{w}}^{\prime}\right)$ with condition (26) and the conditions imposed on $\tilde{\sigma}_{\tilde{t}} \in \tilde{\Omega}\left(\frac{\hat{d}}{4}\right)$, we obtain ${ }^{25}$

$$
\left|\sum_{w \in W} \theta_{j}\left(\tilde{\sigma}_{\bar{t}} ; s_{w^{1}}=s_{\bar{w}}^{\prime} ; w\right) q\left(\tilde{\sigma}_{\bar{t}} ; s_{w^{1}}=s_{\bar{w}}^{\prime} ; w\right)-\sum_{w \in W} \theta^{*}(w) q^{i}\left(s_{w^{1}}=s_{\bar{w}}^{\prime} ; w\right)\right| \geq \frac{\hat{d}}{2} .
$$

Replacing $s_{w^{1}}=s_{\bar{w}}$ by $s_{w^{1}}=s_{\bar{w}}^{\prime}$ and $d$ by $\frac{\hat{d}}{4}$ in the argument used in the proof of Lemma 19 implies that there exists a path $\sigma_{\tilde{t}+\bar{k}\left(\frac{\hat{d}}{4}\right)}=\left(\tilde{\sigma}_{\bar{t}} ; s_{w^{1}}=s_{\bar{w}}^{\prime} ; \ldots s_{w^{\bar{k}}\left(\frac{\hat{d}}{4}\right)}\right)$, which has a probability strictly larger than $\xi\left(\frac{\hat{d}}{4}\right)$ and, thus, belongs to $\tilde{\Xi}\left(\tilde{\sigma}_{\tilde{t}}\right)$, and on which $\left(\tilde{\sigma}_{\tilde{t}} ; s_{w^{1}}=\right.$ $\left.s_{\dot{w}}^{\prime} ; \ldots s_{w^{\bar{k}\left(\frac{\hat{d}}{4}\right)}}\right) \notin[-N ; N]$, violating Lemma 2 . We thus obtain a contradiction to the condition that $\left|\theta_{j}\left(\sigma_{t} ; w^{1}\right)-\theta^{*}\left(w^{1}\right)\right|<\frac{\hat{d}}{4}$ for all $\tilde{\sigma}_{\tilde{t}} \in \tilde{\Omega}\left(\frac{\hat{d}}{4}\right)$ and all $\tilde{t}>t_{0}\left(\frac{\hat{d}}{4}\right)$ established in Lemma 19.

We thus conclude that if $\beta_{i}<\frac{u_{i}^{\prime}\left(m^{\prime}\right)}{u_{i}^{\prime}(\tilde{m})}$, there is no node $\bar{\sigma}_{\bar{t}}$ conditional on which $j$ vanishes with probability 1 , except for a set of initial endowments with measure 0 . Since $i$ and $j$ effectively trade on the same set of assets $W$, the argument for $i$ is symmetric. Q.E.D.

\footnotetext{
${ }^{25}$ Note that $\varepsilon\left(\frac{D}{4}\right)<\frac{D}{4}$ and, hence, $c^{j}\left(\sigma_{t}\right)<\frac{\varepsilon\left(\frac{D}{4}\right)}{2}<\frac{D}{8},\left|\sum_{w \in W} \theta(w) q\left(\sigma_{t} ; w\right)-\sum_{w \in W} \theta^{*}(w) q^{i}(s ; w)\right|<\frac{\varepsilon\left(\frac{D}{4}\right)}{4}<$ $\frac{D}{16}$, and $\left|\theta_{j}\left(\sigma_{t} ; w^{1}\right)-\theta^{*}\left(w^{1}\right)\right|<\frac{D}{4}$.
} 


\section{Proof of Proposition 15}

Let

$$
w^{1} \in \underset{w \in W^{i}}{\arg \max _{s \in w}} \min ^{i}(s)
$$

LEMMA 20: Under the conditions of the proposition, the parameters of the economy satisfy

$$
\begin{aligned}
-\min _{s \in w^{1}} e^{i}(s)-\bar{q} \max _{w \in W^{i}} \min _{s \in w} e^{i}(s) & >\frac{-\max _{w \in W^{i}} \min _{s \in w} e^{i}(s)}{1-\bar{q}} \\
& >-\max _{s \in w^{1}} e^{i}(s)-\underline{q} \min _{w \in W^{i}} \max _{s \in w} e^{i}(s)
\end{aligned}
$$

and

$$
\bar{q} \frac{\max _{s \in w^{1}} e^{i}(s)+\min _{w \in W^{i}} \max _{s \in w} e^{i}(s)}{\max _{s \in w^{1}} e^{i}(s)+\min _{w \in W^{i}} \max _{s \in w} e^{i}(s)-\max _{w \in W^{i}} \min _{s \in w} e^{i}(s)}<1 .
$$

PROOF: Note that

$$
-\min _{s \in w^{1}} e^{i}(s)-\bar{q} \max _{w \in W^{i}} \min _{s \in w} e^{i}(s)>-\frac{\max _{w \in W^{i}} \min _{s \in w} e^{i}(s)}{1-\bar{q}}
$$

is equivalent to $\bar{q}^{2} \max _{w \in W^{i}} \min _{s \in w} e^{i}(s)>0$, which is always satisfied.

Since $\max _{w \in W^{i}} \min _{s \in w} e^{i}(s)<(1-\underline{q})(1-\bar{q}) \min _{w \in W^{i}} \max _{s \in w} e^{i}(s)$,

$$
\begin{aligned}
& -\max _{w \in W^{i}} \min _{s \in w} e^{i}(s)>-(1-\underline{q})(1-\bar{q}) \min _{w \in W^{i}} \max _{s \in w} e^{i}(s) \text { or } \\
& \frac{-\max _{w \in W^{i}} \min _{s \in w} e^{i}(s)}{1-\bar{q}}>-\max _{s \in w^{1}} e^{i}(s)-\underline{q} \min _{w \in W^{i}} \max _{s \in w} e^{i}(s)
\end{aligned}
$$

and we obtain (30).

Since $\underline{q}<1$, the second inequality in (30) then implies

$$
\frac{-\max _{w \in W^{i}} \min _{s \in w} e^{i}(s)}{1-\bar{q}}>-\max _{s \in w^{1}} e^{i}(s)-\min _{w \in W^{i}} \max _{s \in w} e^{i}(s),
$$

which is equivalent to

$$
0<\left[(1-\bar{q}) \max _{s \in w^{1}} e^{i}(s)+(1-\bar{q}) \min _{w \in W^{i}} \max _{s \in w} e^{i}(s)-\max _{w \in W^{i}} \min _{s \in w} e^{i}(s)\right]
$$

and, thus, to (31). 
Choose $\varepsilon$ such that $\varepsilon<\tilde{m}$ and by (30) such that

$$
\frac{-\max _{w \in W^{i}} \min _{s \in w} e^{i}(s)}{1-\bar{q}}>-\max _{s \in w^{1}} e^{i}(s)-\underline{q}_{w \in W^{i}} \max _{s \in w} e^{i}(s)+\varepsilon(1-\underline{q}) .
$$

Let $\bar{k}$ be the smallest natural number such that

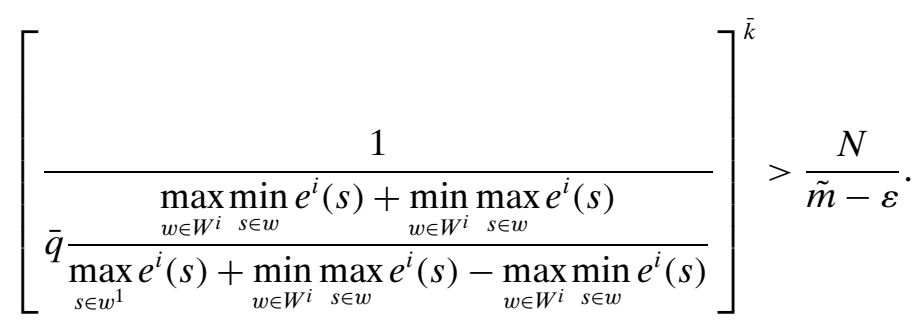

By (31), $\bar{k}$ is finite and since, clearly

$$
\frac{\max _{w \in W^{i}} \min _{s \in w} e^{i}(s)+\min _{w \in W^{i}} \max _{s \in w} e^{i}(s)}{\max _{s \in w^{1}} e^{i}(s)+\min _{w \in W^{i}} \max _{s \in w} e^{i}(s)-\max _{w \in W^{i}} \min _{s \in w} e^{i}(s)}>1,
$$

(33) implies

$$
\left[\begin{array}{l}
{\left[\frac{1}{\bar{q}}\right]^{\bar{k}}>\frac{N}{\tilde{m}-\varepsilon} \text { and }} \\
\left.\bar{q} \frac{1}{\max _{s \in w^{1}} e^{i}(s)+\max _{w \in W^{i}} e^{i}(s)+\min _{w \in W^{i}} \max _{s \in w} e^{i}(s)-\min _{s \in w^{1}} e^{i}\left(s_{w^{1}}\right)}\right]^{\bar{k}}>\frac{N}{\tilde{m}} .
\end{array}\right.
$$

Let $^{26} \xi \in\left(0 ; \underline{\pi}^{\bar{k}+2}\right)$.

Suppose in a manner of contradiction that $i$ vanishes a.s. conditional on node $\bar{\sigma}_{\bar{t}}$. Then there exists a $\hat{t}(\varepsilon ; \xi)$ such that

$$
\pi\left(\sigma \mid \sigma_{\bar{t}}=\bar{\sigma}_{\bar{t}}, c^{j}\left(\sigma_{t}\right)<\varepsilon \text { for all } t \geq \hat{t}(\varepsilon ; \xi)\right)>1-\xi .
$$

Since for any $\tilde{t}>\hat{t}(\varepsilon ; \xi)$, the number of paths $\sigma_{\tilde{t}}$ such that $\sigma_{\bar{t}}=\bar{\sigma}_{\bar{t}}$ is finite, it follows that there is at least one path $\tilde{\sigma}_{\tilde{t}}$ such that

$$
\pi\left(\Xi\left(\tilde{\sigma}_{\tilde{t}} ; \varepsilon ; \xi\right)\right)=\pi\left(\sigma \mid \sigma_{\bar{t}}=\bar{\sigma}_{\bar{t}}, \sigma_{\tilde{t}}=\tilde{\sigma}_{\tilde{t}}, c^{j}\left(\sigma_{t}\right)<\varepsilon \text { and for all } t \geq \tilde{t}\right)>1-\xi .
$$

Denote by $\tilde{\Omega}(\varepsilon ; \xi)$ the set of all nodes $\tilde{\sigma}_{\tilde{t}}$ satisfying these properties. Note that as in the proof of Proposition $14, \sigma_{t} \in \tilde{\Omega}(\varepsilon ; \xi)$ occurs i.o. a.s. on every path such that $\sigma_{\bar{t}}=$ $\bar{\sigma}_{\bar{t}}$.

Q.E.D.

\footnotetext{
${ }^{26}$ Clearly, the definition of both $\xi$ and $\bar{k}$ depends on $\varepsilon$. However, since the value of $\varepsilon$ will remain fixed for this proof, this dependence is omitted in the notation for brevity.
} 
LEMMA 21: For any $\tilde{\sigma}_{\hat{t}} \in \tilde{\Omega}(\varepsilon ; \xi)$,

$$
\theta_{i}\left(\tilde{\sigma}_{\tilde{t}} ; w^{1}\right)<-\max _{s \in w^{1}} e^{i}(s)+\varepsilon-\underline{q}\left[\min _{w \in W^{i}} \max _{s \in w} e^{i}(s)-\varepsilon\right]
$$

must hold.

PROOF: Suppose in a manner of contradiction that $\theta_{i}\left(\tilde{\sigma}_{\tilde{t}} ; w^{1}\right) \geq-\max _{s \in w^{1}} e^{i}(s)+\varepsilon-$ $\underline{q}\left[\min _{w \in W^{i}} \max _{s \in w} e^{i}(s)-\varepsilon\right]$ for some $\tilde{\sigma}_{\bar{t}} \in \tilde{\Omega}(\varepsilon ; \xi)$. Given $\tilde{\sigma}_{\tilde{t}}, w^{1}$ occurs with probability at least $\underline{\pi}>\xi$. According to the budget constraint of agent $i$, and since $i$ 's consumption is lower than $\varepsilon$,

$$
\sum_{w \in W^{i}} \theta_{i}\left(\tilde{\sigma}_{\tilde{t}} ; s_{w^{1}} ; w\right) q\left(\tilde{\sigma}_{\tilde{t}} ; s_{w^{1}} ; w\right) \geq \theta_{i}\left(\tilde{\sigma}_{\tilde{t}} ; w^{1}\right)+e^{i}\left(s_{w^{1}}\right)-\varepsilon .
$$

Since $q \leq \sum_{w \in W^{i}} q\left(\tilde{\sigma}_{\tilde{t}} ; s_{w^{1}} ; w\right) \leq \bar{q}<1$, it follows that there exists a state realization $s_{w^{1}}$ and $w^{2}$ such that

$$
\theta_{i}\left(\tilde{\sigma}_{\tilde{t}} ; s_{w^{1}} ; w^{2}\right) \geq \frac{\theta_{i}\left(\tilde{\sigma}_{\tilde{t}} ; w^{1}\right)+e^{i}\left(s_{w^{1}}\right)-\varepsilon}{\sum_{w \in W^{i}} q\left(\tilde{\sigma}_{\tilde{t}} ; s_{w^{1}} ; w\right)}>-\min _{w \in W^{i}} \max _{s \in w} e^{i}(s)+\varepsilon
$$

and $\left(\tilde{\sigma}_{\tilde{t}} ; s_{w^{1}}\right)$ belongs to the set $\Xi\left(\tilde{\sigma}_{\ddot{t}} ; \varepsilon ; \xi\right)$ in (34).

By a similar argument, there exists a state realization $s_{w^{2}} \in w^{2}$ and a $w^{3}$ such that $\left(\tilde{\sigma}_{\tilde{t}} ; s_{w^{1}} ; s_{w^{2}}\right)$ occurs with conditional probability of at least $\underline{\pi}^{2}>\xi$ and, hence, belongs to $\Xi\left(\tilde{\sigma}_{\tilde{t}} ; \varepsilon ; \xi\right)$ and

$$
\theta_{i}\left(\tilde{\sigma}_{\tilde{t}} ; s_{w^{1}} ; w^{2} ; w^{3}\right) \geq \frac{\theta_{i}\left(\tilde{\sigma}_{\tilde{t}} ; s_{w^{1}} ; w^{2}\right)+e^{i}\left(s_{w^{2}}\right)-\varepsilon}{\sum_{w \in W^{i}} q\left(\tilde{\sigma}_{\tilde{t}} ; s_{w^{1}} ; s_{w^{2}} ; w\right)}>0 .
$$

Then there exists $s_{w^{3}} \in w^{3}$ and $w^{4}$ such that $\left(\tilde{\sigma}_{\tilde{t}} ; s_{w^{1}} ; s_{w^{2}}\right)$ occurs with conditional probability of at least $\underline{\pi}^{3}>\xi$ and, hence, belongs to $\Xi\left(\tilde{\sigma}_{i} ; \varepsilon ; \xi\right)$ and

$$
\theta_{i}\left(\tilde{\sigma}_{\bar{t}} ; s_{w^{1}} ; w^{2} ; w^{3}\right) \geq \frac{e^{i}\left(s_{w^{3}}\right)-\varepsilon}{\sum_{w \in W^{i}} q\left(\tilde{\sigma}_{\bar{t}} ; s_{w^{1}} ; s_{w^{2}} ; s_{w^{3}} ; w\right)} .
$$

We obtain by induction that there exists a sequence $w^{1} \ldots w^{\bar{k}+2}$ such that $\left(\tilde{\sigma}_{\tilde{t}} ; s_{w^{1}} \ldots s_{w^{\bar{k}+2}}\right)$ occurs with conditional probability at least $\pi\left(s_{w^{1}} \ldots s_{w^{\bar{k}+1}}\right) \geq \underline{\pi}^{\bar{k}+2}>\xi$ and, hence, belongs to $\Xi\left(\tilde{\sigma}_{\tilde{t}} ; \varepsilon ; \xi\right)$ and

$$
\begin{aligned}
& \theta_{i}\left(\tilde{\sigma}_{\bar{t}} ; s_{w^{1}} ; s_{w^{2}} \ldots s_{w^{\bar{k}+2}} ; w^{\bar{k}+3}\right) \\
& \quad>\frac{\theta_{i}\left(\tilde{\sigma}_{\bar{t}} ; s_{w^{1}} ; \ldots s_{w^{\bar{k}+1}} ; w^{\bar{k}+2}\right)+e^{i}\left(s_{w^{\bar{k}+2}}\right)-\varepsilon}{\sum_{w \in W^{i}} q\left(\tilde{\sigma}_{\bar{t}} ; s_{w^{1}} ; \ldots s_{w^{\bar{k}+2}} ; w\right)}
\end{aligned}
$$




$$
\begin{aligned}
& >\frac{\theta_{i}\left(\tilde{\sigma}_{\bar{t}} ; s_{w^{1}} ; \ldots s_{w^{\bar{k}}} ; w^{\bar{k}+1}\right)+e^{i}\left(s_{w^{\bar{k}+1}}\right)-\varepsilon}{\sum_{w \in W^{i}} q\left(\tilde{\sigma}_{\tilde{t}} ; s_{w^{1}} ; \ldots s_{w^{\bar{k}+1}} ; w\right) \sum_{w \in W^{i}} q\left(\tilde{\sigma}_{\tilde{t}} ; s_{w^{1}} ; \ldots s_{w^{\bar{k}+2}} ; w\right)}+\frac{e^{i}\left(s_{w^{\bar{k}+2}}\right)-\varepsilon}{\sum_{w \in W^{i}} q\left(\tilde{\sigma}_{\tilde{t}} ; s_{w^{1}} ; \ldots s_{w^{\bar{k}+2}} ; w\right)} \\
& \ldots \\
& \geq \frac{e_{i}\left(s_{w^{3}}\right)-\varepsilon}{\bar{q}^{\bar{k}}} \geq \frac{\tilde{m}-\varepsilon}{\bar{q}^{\bar{k}}}>N .
\end{aligned}
$$

Since $\theta_{j}\left(\tilde{\sigma}_{\tilde{t}} ; s_{w^{1}} \ldots s_{w^{\bar{k}+2}} ; w^{\bar{k}+3}\right)>N$ contradicts Lemma 2, we obtain the desired result. Q.E.D.

LEMMA 22: For any $\tilde{\sigma}_{\tilde{t}} \in \tilde{\Omega}(\varepsilon ; \xi)$,

$$
\theta_{i}\left(\tilde{\sigma}_{\tilde{t}} ; w^{1}\right) \geq-\max _{s \in w^{1}} e^{i}(s)+\varepsilon-\underline{q}\left[\min _{w \in W^{i}} \max _{s \in w} e^{i}(s)-\varepsilon\right]
$$

must hold.

PRoOF: Suppose in a manner of contradiction that for some $\tilde{\sigma}_{i} \in \tilde{\Omega}(\varepsilon ; \xi), \theta_{i}\left(\tilde{\sigma}_{\tilde{t}} ; w^{1}\right) \leq$ $-\max _{s \in w^{1}} e^{i}(s)+\varepsilon-q\left(\min _{w \in W^{i}} \max _{s \in w} e^{i}(s)-\varepsilon\right)$ and, hence, by (30) and (32),

$$
\theta_{i}\left(\tilde{\sigma}_{\tilde{t}} ; w^{1}\right)<\frac{-\max _{w \in W^{i}} \min _{s \in w} e^{i}(s)}{1-\bar{q}}
$$

and

$$
\theta_{i}\left(\tilde{\sigma}_{\tilde{t}} ; w^{1}\right)<-\min _{s \in w^{1}} e^{i}(s)-\bar{q} \max _{w \in W^{i}} \min _{s \in w} e^{i}(s)=-(1-\bar{q}) \max _{w \in W^{i}} \min _{s \in w} e^{i}(s) .
$$

Hence, there is a state realization $s_{w^{1}}$, which occurs with probability at least $\underline{\pi}>\xi$ and $w^{2}$, such that the budget constraint of agent $i$ implies

$$
\theta_{i}\left(\tilde{\sigma}_{i} ; s_{w^{1}} ; w^{2}\right) \leq \frac{\theta_{i}\left(\tilde{\sigma}_{\tilde{t}} ; w^{1}\right)+\min _{s^{1} \in w^{1}} e^{i}\left(s_{w^{1}}\right)}{\bar{q}}
$$

and $\left(\tilde{\sigma}_{\tilde{t}} ; s_{w^{1}}\right)$ belongs to $\Xi\left(\tilde{\sigma}_{\tilde{t}} ; \varepsilon ; \xi\right)$.

Let $\tilde{q}_{1}$ be defined as

$$
\theta_{i}\left(\tilde{\sigma}_{\tilde{t}} ; s_{w^{1}} ; w^{2}\right)=\frac{\theta_{i}\left(\tilde{\sigma}_{\tilde{t}} ; w^{1}\right)}{\tilde{q}_{1}}
$$

Note that

$$
\begin{aligned}
& \frac{\theta_{i}\left(\tilde{\sigma}_{\tilde{t}} ; w^{1}\right)}{\tilde{q}_{1}}<\frac{\theta_{i}\left(\tilde{\sigma}_{\tilde{t}} ; w^{1}\right)+\min _{s_{w^{1}} \in w^{1}} e^{i}\left(s_{w^{1}}\right)}{\bar{q}}, \\
& \tilde{q}_{1}<\bar{q} \frac{\theta_{i}\left(\tilde{\sigma}_{\bar{t}} ; w^{1}\right)}{\theta_{i}\left(\tilde{\sigma}_{\tilde{t}} ; w^{1}\right)+\min _{s_{w^{1}} \in w^{1}} e^{i}\left(s_{w^{1}}\right)}<1,
\end{aligned}
$$

where the last inequality follows from (35). 
Note further that

$$
\bar{q} \frac{\theta_{i}\left(\tilde{\sigma}_{\tilde{t}} ; w^{1}\right)}{\theta_{i}\left(\tilde{\sigma}_{\tilde{t}} ; w^{1}\right)+\min _{s_{w^{1}} \in w^{1}} e^{i}\left(s_{w^{1}}\right)}<\bar{q} \frac{\max _{s \in w^{1}} e^{i}(s)+\min _{w \in W^{i}} \max _{s \in w} e^{i}(s)}{\max _{s \in w^{1}} e^{i}(s)+\min _{w \in W^{i}} \max _{s \in w} e^{i}(s)-\min _{s^{1} \in w^{1}} e^{i}\left(s_{w^{1}}\right)}<1,
$$

where the second inequality was shown in (31).

We conclude that

$$
\theta_{i}\left(\tilde{\sigma}_{i} ; s_{w^{1}} ; w^{2}\right)<\theta_{i}\left(\tilde{\sigma}_{i} ; w^{1}\right)<-\frac{\max _{w \in W^{i}} \min _{s_{w} \in w} e^{i}\left(s_{w}\right)}{1-\bar{q}} .
$$

Hence, there exists an $s_{w}^{2}$ and $w^{3}$ such that, according to $i$ 's budget constraint,

$$
\theta_{i}\left(\tilde{\sigma}_{\tilde{t}} ; s_{w^{1}} ; s_{w^{2}} ; w^{3}\right) \leq \frac{\theta_{i}\left(\tilde{\sigma}_{\tilde{t}} ; s_{w^{1}} ; w^{2}\right)+\min _{s_{w}^{2} \in w^{2}} e^{i}\left(s_{w}^{2}\right)}{\bar{q}}
$$

and the conditional probability of $\left(\tilde{\sigma}_{t} ; s_{w^{1}} ; s_{w^{2}}\right)$ is at least $\underline{\pi}^{2}>\xi$, implying that it belongs to $\Xi\left(\tilde{\sigma}_{\tilde{t}} ; \varepsilon ; \xi\right)$. Define $\tilde{q}_{2}$ as

$$
\theta_{i}\left(\tilde{\sigma}_{\tilde{t}} ; s_{w^{1}} ; s_{w^{2}} ; w^{3}\right)=\frac{\theta_{i}\left(\tilde{\sigma}_{t} ; s_{w^{1}} ; w^{2}\right)}{\tilde{q}_{2}}
$$

and note that

$$
\tilde{q}_{2} \leq \bar{q} \frac{\theta_{i}\left(\tilde{\sigma}_{\tilde{t}} ; s_{w^{1}} ; w^{2}\right)}{\theta_{i}\left(\tilde{\sigma}_{\tilde{t}} ; s_{w^{1}} ; w^{2}\right)+\min _{s_{w}^{2} \in w^{2}} e^{i}\left(s_{w}^{2}\right)}<\bar{q} \frac{\theta_{i}\left(\tilde{\sigma}_{\tilde{t}} ; w^{1}\right)}{\theta_{i}\left(\tilde{\sigma}_{\tilde{t}} ; w^{1}\right)+\min _{w^{1} \in w^{1}} e^{i}\left(s_{w^{1}}\right)}<1 .
$$

Proceeding iteratively, we conclude that there exists a sequence $s_{w^{1}} \ldots s_{w^{\bar{k}}}$ with conditional probability at least $\pi\left(s_{w^{1}} \ldots s_{w^{\bar{k}}}\right) \geq \underline{\pi}^{\bar{k}}>\xi$ so that $\sigma_{\tilde{t}+\bar{k}}=\left(\tilde{\sigma}_{\tilde{t}} ; s_{w^{1}} \ldots s_{w^{\bar{k}+2}}\right)$ belongs to $\Xi\left(\tilde{\sigma}_{\tilde{t}} ; \varepsilon ; \xi\right)$ and a $w^{\bar{k}+1}$ such that

$$
\begin{aligned}
& \theta_{i}\left(\tilde{\sigma}_{\bar{t}} ; s_{w^{1}} \ldots s_{w^{\bar{k}}} ; w^{\bar{k}+1}\right)<\frac{\theta_{i}\left(\tilde{\sigma}_{\tilde{t}} ; w^{1}\right)}{\left[\bar{q} \frac{\max _{s \in w^{1}} e^{i}(s)+\min _{w \in W^{i}} \max _{s \in w} e^{i}(s)}{\left[\max ^{1} e^{i}(s)+\min _{w \in W^{i}} \max _{s \in w} e^{i}(s)-\min _{s^{1} \in w^{1}} e^{i}\left(s_{w^{1}}\right)\right.}\right]^{\bar{k}}}
\end{aligned}
$$

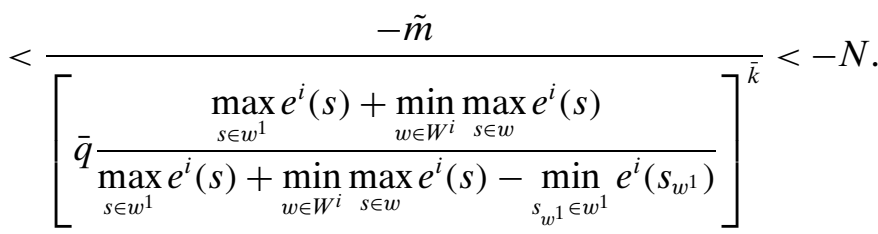

However, $\theta_{i}\left(\tilde{\sigma}_{\bar{t}} ; s_{w^{1}} \ldots s_{w^{\bar{k}}} ; w^{\bar{k}+1}\right)<-N$ contradicts Lemma 2, thus establishing the result of the lemma.

Q.E.D.

Combining the results of Lemmata 21 and 22, we conclude that there is no node $\bar{\sigma}_{\bar{t}}$ conditional on which agent $i$ a.s. vanishes. 


\section{REFERENCES}

Alchian, A. A. (1950): "Uncertainty, Evolution and Economic Theory," Journal of Political Economy, 58, 211-221. [1697]

Allen, F., AND D. Gale (1994): "Limited Market Participation and Volatility of Asset Prices," American Economic Review, 84, 933-955. [1699]

ARAUJO, A., J. FAJARDO, AND M. R. PASCOA (2005): “Endogeneous Colatteral," Journal of Mathematical Economics, 41, 439-462. [1698]

ARrow, K. J., AND G. DeBreU (1954): “Existence of an Equilibrium for a Competitive Economy,” Econometrica, 22, 265-290. [1700]

AumAnN, R. (1976): "Agreeing to Disagree," The Annals of Statistics, 4, 1236-1239. [1694]

BALASKO, Y., D. CASS, AND K. SHELl (1995): "Market Participation and Sunspot Equilibria," Review of Economic Studies, 62, 491-512. [1698]

BAQUeroy, G., J. TER HorSTZ, AND M. VerbeeK (2005): "Survival, Look-Ahead Bias and the Persistence in Hedge Fund Performance," Journal of Financial and Quantitative Analysis, 40, 493-517. [1716]

BASAC, S., AND D. CuOCO (1998): "An Equilibrium Model With Restricted Stock Market Participation," The Review of Financial Studies, 11, 309-341. [1698]

BeKer, P., AND S. K. ChatTopadhyAY (2010): "Consumption Dynamics in General Equilibrium: A Characterization When Markets Are Incomplete,” Journal of Economic Theory, 145, 2133-2185. [1696,1697,1716, 1720]

BEWLEY, T. (1972): "Existence of Equilibria in Economies With Infinitely Many Commodities," Journal of Economic Theory, 4, 514-540. [1703,1726,1727,1743]

Blume, L., AND D. EASLEY (2006): "If You Are so Smart, Why Aren't You Rich? Belief Selection in Complete and Incomplete Markets," Econometrica, 74, 929-966. [1693,1695,1697,1702,1707,1715,1716,1721,1732]

Blume, L., T. Cogley, D. EASley, T. SARgent, AND V. Tsyrennikov (2018): "A Case for Incomplete Markets," Journal of Economic Theory, 178, 191-221. [1694,1698]

BRAv, A., G. M. Constantinides, And C. C. GeCZy (2002): "Asset Prices With Heterogeneous Consumers and Limited Participation," Journal of Political Economy, 110, 793-824. [1699]

Brunnermeier, M. K., A. SimseK, AND W. Xiong (2014): "A Welfare Criterion for Models With Distorted Beliefs," Quarterly Journal of Economics, 129, 1753-1797. [1698]

CALVET, L., M. GONZALEZ-EIRAS, AND P. SODINI (2004): "Financial Innovation, Market Participation and Asset Prices," Journal of Financial and Quantative Analysis, 39, 432-459. [1699]

CAO, H. H., T. WANG, AND H. H. ZHANG (2005): "Model Uncertainty, Limited Market Participation, and Asset Prices," The Review of Financial Studies, 18, 1219-1251. [1699]

Choquet, G. (1956): "Existence et unicité des représentations intégrales au moyen des points extrêmaux dans les cônes convexes," Séminaire Bourbaki, 4, 33-47. [1695]

COGLEY, T., T. SARgENT, AND V. TsyrenNiKOV (2014): "Wealth Dynamics in a Bond Economy With Heterogeneous Beliefs," Economic Journal, 124, 1-30. [1697]

COURY, T., AND E. ScIUBBA (2012): "Belief Heterogeneity and Survival in Incomplete Markets," Economic Theory, 49, 37-58. [1696,1697,1716]

DeBREU, G. (1962): "New Concepts and Techniques for Equilibrium Analysis," International Economic Review, 3, 257-273. [1727]

DETEMPLE, J., AND A. SERRAT (2003): "Dynamic Equilibrium With Liquidity Constraints," Review of Financial Studies, 16, 597-629. [1698]

EASLEY, D., AND M. O'HARA (2009): "Ambiguity and Non-Participation: The Role of Regulation,” The Review of Financial Studies, 22, 1817-1843. [1699]

EICHHORN, D., F. GUPTA, AND E. STUBBS (1998): "Using Constraints to Improve the Robustness of Asset Allocation," The Journal of Portfolio Management, 24, 41-48. [1715]

ERrunZA, V., AND E. LOSQ (1985): “International Asset Pricing Under Mild Segmentation: Theory and Test," Journal of Finance, 15, 105-124. [1698]

(1989): "Capital Flow Controls, International Asset Pricing and Investors' Welfare: A MultiCountry Framework,” Journal of Finance, 19, 1025-1037. [1698,1699]

French, K., AND J. POTERBA (1991): "Agent Diversification and International Equity Markets,” American Economic Review, 81, 222-226. [1698,1700]

FRIEDMAN, M. (1953): Essays in Positive Economics. Chicago: University of Chicago Press. [1697]

GEANAKOPLOS, J., AND W. ZAME (2014): "Collateral Equilibrium I, A Basic Framework," Economic Theory, 56, 443-492. [1698]

GilboA, I., L. SAmUelson, AND D. Schmeidler (2014): “No-Betting Pareto Dominance,” Econometrica, 82, 1405-1442. [1698] 
Goodhart, C., N. Romanidis, D. P. Tsomokos, AND M. ShubiK (2016): "Macro-Modelling, Default and Money," Said Business School Research Papers 2016-18, Available at SSRN, https://ssrn.com/abstract= 2788772 or http://dx.doi.org/10.2139/ssrn.2788772. [1698]

GotTARdi, P., AND F. Kubler (2015): "Dynamic Competitive Economies With Complete Markets and Collateral Constraints," Review of Economic Studies, 82, 1119-1153. [1698]

GRANT, S., AND J. Quiggin (2013): "Bounded Awareness, Heuristics and the Precautionary Principle," Journal of Economic Behavior and Organization, 93, 17-31. [1712]

Grossman, S. J., AND J. E. STIGLiTZ (1980): “On the Impossibility of Informationally Efficient Markets," American Economic Review, 70, 393-408. [1715]

Guerdjikova, A., AND J. Quiggin (2019): "Supplement to 'Market Selection With Differential Financial Constraints'," Unpublished manuscript, University of Grenoble Alpes, Available at https://hal. archives-ouvertes.fr/hal-02099920. [1695,1701,1717]

Guevenen, F. (2009): “A Parsimonious Model for Asset Pricing,” Econometrica, 77, 1711-1750. [1699]

Guiso, L., AND T. JAPPELli (2005): “Awareness and Stock Market Participation,” Review of Finance, 9, 537567. [1694]

Guo, H. (2004): "Limited Stock Market Participation and Asset Prices in a Dynamic Economy," Journal of Financial and Quantative Analysis, 39, 495-516. [1698,1699]

Hall, R. L., AND C. J. Hitch (1939): "Price Theory and Business Behavior," Oxford Economic Papers, os-2, 12-45. [1697]

KARNI, E., AND M.-L. VIERø (2013): “'Reverse Bayesianism': A Choice-Based Theory of Growing Awareness," American Economic Review, 103, 2790-2810. [1712]

- (2017): "Awareness of Unawareness: A Theory of Decision-Making in the Face of Ignorance," Journal of Economic Theory, 168, 301-328. [1712]

KehoE, T. J., AND D. K. LeVINE (2001): "Liquidity Constrained Markets versus Debt Constrained Markets," Econometrica, 69, 575-598. [1698]

Kendall, D. G. (1962): "Simplexes and Vector Lattices," Journal of the London Mathematical Society, 37, 365-371. [1695]

KIYOTAKI, N., AND J. MOORE (2005): “2002 Lawrence R. Klein Lecture: Liquidity and Asset Prices,” International Economic Review, 46, 317-349. [1698]

Kountzakis, C., AND I. A. POlYRAKIS (2006): “The Completion of Security Markets,” Decisions in Economics and Finance, 29, 1-21. [1695]

KREBS, T. (2004): "Non-Existence of Recursive Equilibria on Compact State Spaces When Markets Are Incomplete," Journal of Economic Theory, 115, 134-150. [1726,1743]

MaGILL, M., AND M. QuinZII (1994): “Infinite Horizon Incomplete Markets,” Econometrica, 62, 853-880. $[1717-1719,1726,1744]$

MARINACCI, M. (1994): “Genericity: A Measure-Theoretic Analysis,” Unpublished Manuscript, Northwestern University. [1725]

MAssari, F. (2013): “Comment on if You're so Smart, Why Aren't You Rich? Belief Selection in Complete and Incomplete Markets," Econometrica, 81, 849-851. [1697]

MiNARDI, S., AND A. SAVOCHKIN (2016): "Subjective Contingencies and Limited Bayesian Updating," HEC Paris Research Paper No. ECO-SCD-2017-1203, Available at SSRN https://ssrn.com/abstract=2962260 or http://dx.doi.org/10.2139/ssrn.2962260. [1702]

POLYRAKIS, I. A. (1999): "Minimal Lattice Subspaces," Transactions of the American Mathematical Society, 351, 4183-4203. [1695]

Posner, E., AND E. G. WeYl (2013): “Benefit-Cost Analysis for Financial Regulation,” American Economic Review, 103, 393-397. [1698]

Quiggin, J., AND H. SIDDIQI (2015): "Differential Awareness, Financial Innovation and Financial Crises," Unpublished Manuscript, University of Queensland, Available at SSRN https://ssrn.com/abstract =2483445 or http://dx.doi.org/10.2139/ssrn.2483445. [1694]

Rudin, W. (1983): "Well-Distributed Measurable Sets," The American Mathematical Monthly, 90, 41-42. [1725]

SAndroni, A. (2000): "Do Markets Favor Agents Able to Make Accurate Predictions?" Econometrica, 68, 1303-1334. [1693,1697]

(2005): "Market Selection When Markets Are Incomplete," Journal of Mathematical Economics, 41, 91-104. [1694,1695,1703,1707,1716]

SCHIPPER, B. (2016): “The Unawareness Bibliography,”http://www.econ.ucdavis.edu/faculty/schipper/unaw. htm. [1712]

ScIUBBA, E. (2005): “Asymmetric Information and Survival in Financial Markets,” Economic Theory, 25, 353379. [1715] 
SimseK, A. (2013): "Financial Innovation and Portfolio Risk," American Economic Review, 103, 398-401. [1715]

STIGLER, G. J. (1958): “The Economies of Scale,” Journal of Law and Economics, 1, 54-71. [1697]

WEYL, E. G. (2007): "Is Arbitrage Socially Beneficial?" Unpublished Manuscript, Princeton University, Available at SSRN https://ssrn.com/abstract=1324423 or http://dx.doi.org/10.2139/ssrn.1324423. [1697]

ZHOU, J. (2015): "Household Stockholding Behavior During the Great Financial Crisis," Bank of Canada Working Paper 2015-15, Available at http://hdl.handle.net/10419/123769. [1698]

Co-editor Itzhak Gilboa handled this manuscript.

Manuscript received 12 May, 2017; final version accepted 10 April, 2019; available online 10 April, 2019. 